\title{
A Highly Efficient Base-Metal Catalyst: Chemoselective Reduction of Imines to Amines Using An Abnormal-NHC-Fe(0) Complex
}

Mrinal Bhunia ${ }^{[\mathrm{a}]}$ Pradip Kumar Hota, ${ }^{[\mathrm{a}]}$ Gonela Vijaykumar, ${ }^{[\mathrm{a}]}$ Debashis Adhikari $^{[\mathrm{b}]}$ and Swadhin K. $\operatorname{Mandal}^{[\mathrm{a}] *}$

${ }^{[a]}$ Department of Chemical Sciences, Indian Institute of Science Education and Research, Kolkata, Mohanpur-741246, India;

${ }^{[b]}$ Department of Chemical Sciences, Indian Institute of Science Education and Research Mohali, SAS Nagar-140306, India.

* E-mail: swadhin.mandal@iiserkol.ac.in 
Electronic Supporting Information

I. Experimental ..........................................................................................................................

II. General considerations.......................................................................................................

III. Synthesis of complexes...............................................................................................S3

IV. Optimisation study for the reduction of N-benzylideneanilinewith 1 as the

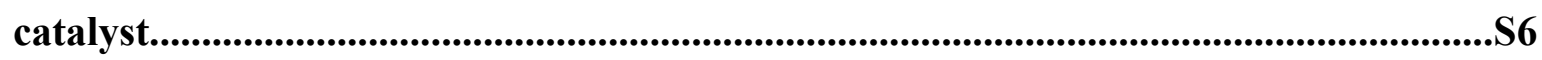

V.General procedure for catalytic hydrosilylation of aldimines to secondary amines by

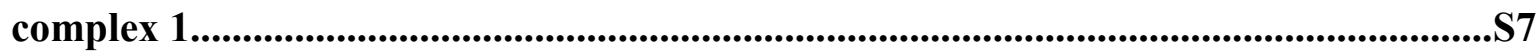

VI. Characterization data for secondary amines....................................................................S7

VII. Optimisation study for the reduction of4-methyl-N-(1-phenylethylidene)aniline....S14

VIII. General procedure for catalytic hydrosilylation of ketimines to secondary amines and characterization data.......................................................................................................S15 and S16

IX.General procedure for catalytic hydrosilylation of imines containing sugar moieties to secondary amines and characterization data..............................................................S18 and S19

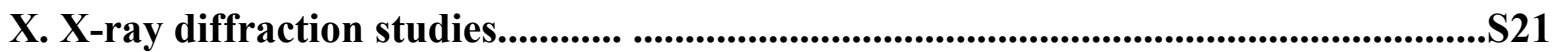

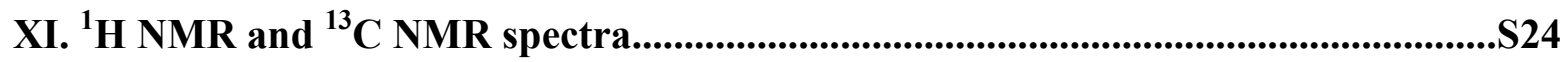

XII. IR spectrum of complex 1 and complex 9....................................................S94 and S95

XIII. Computational details.........................................................................................................596

XIV. References......................................................................................................................................59 


\section{Experimental}

\section{General considerations}

All manipulations were performed under a dry and oxygen free atmosphere $\left(\mathrm{N}_{2}\right)$ usingstandardSchlenk techniques or inside a glovebox maintained below $0.1 \mathrm{ppm}$ of $\mathrm{O}_{2}$ and $\mathrm{H}_{2}$ Olevel, utilizing glasswares that were oven-dried $\left(130{ }^{\circ} \mathrm{C}\right)$ and evacuated while hot prior touse. All solvents were distilled from $\mathrm{Na}$ /benzophenone prior to use.Allotherchemicals were purchased from commercial sourcesand used as received. The HRMS data was obtained using a Finnigan MAT 8230 instrument. Elemental analyses were carried out using aPerkin-Elmer 2400 $\mathrm{CHN}$ analyser and samples were prepared by keeping under reduced pressure $\left(10^{-2} \mathrm{mbar}\right)$ for overnight. The melting point was measured in a sealed glass tube on a Büchi B-540 melting point apparatus and was uncorrected. Analytical TLC was performed on a Merck $60 \mathrm{~F}_{254}$ silica gel plate $(0.25 \mathrm{~mm}$ thickness). Column chromatography was performed on Merck 60silica gel (100-200 mesh). Deuterated benzene waspurchased from Sigma-Aldrich and dried by sodium potassium alloy and stored over 4 Åmolecular sieves prior to use.NMR spectra were recorded on a JEOL ECS $400 \mathrm{MHz}$ spectrometerand on a Bruker Avance III $500 \mathrm{MHz}$ spectrometer. All chemical shifts were reported in ppmusingtetramethylsilane as a reference. Chemical shifts $(\delta)$ downfield from the reference standardwere assigned positive values.

\section{Synthesis of (aNHC)iron-tetracarbonyl complex :}

Synthesis of the complex 1; $(\boldsymbol{a N H C}) \mathrm{Fe}(\mathrm{CO})_{4}$ : In a glovebox, an oven dried $50 \mathrm{~mL}$ Schlenkflask was charged with $\mathrm{Fe}_{2}(\mathrm{CO})_{9}(200 \mathrm{mg}, 0.55 \mathrm{mmol})$ and $a \mathrm{NHC}(541 \mathrm{mg}, 1 \mathrm{mmol})$ and then dry toluene $(25 \mathrm{~mL})$ was added via cannula at $25{ }^{\circ} \mathrm{C}$ in argon atmosphere. The reaction mixture was stirred overnight and during the course of reaction,color changed to yellowish red. Then the reaction mixture was filtered through a celite pad and concentrated the reaction mixture to ca. $8 \mathrm{~mL}$. Storage of this reaction mixture at $-20{ }^{\circ} \mathrm{C}$ for $2-3$ days afforded yellow color crystals (370 mg, $0.52 \mathrm{mmol}, 52 \%)$.

M.P : $208.3^{\circ} \mathrm{C}$. 
${ }^{1} \mathrm{H}$ NMR $\left(\mathrm{C}_{6} \mathrm{D}_{6}, 500 \mathrm{MHz}, 298 \mathrm{~K}\right): \delta 7.85$ (d, 2H, $\left.J=7.5 \mathrm{~Hz}\right), 7.36(\mathrm{t}, 1 \mathrm{H}, J=7.5 \mathrm{~Hz}), 7.15$ (s, 2H), $7.07(\mathrm{t}, 2 \mathrm{H}, J=8 \mathrm{~Hz}), 6.95-6.91(\mathrm{~m}, 2 \mathrm{H}), 6.81(\mathrm{~d}, 2 \mathrm{H}, J=7.5 \mathrm{~Hz}), 6.76(\mathrm{~d}, 2 \mathrm{H}, J=8 \mathrm{~Hz})$, 6.57-6.54 (m, 1H), 6.51-6.48 (m, 2H), 2.99 (sept, 2H, $J=6.5 \mathrm{~Hz}), 2.93$ (sept, 2H, $J=6.5 \mathrm{~Hz}$ ), $1.60(\mathrm{~d}, 6 \mathrm{H}, J=6.5 \mathrm{~Hz}), 0.99(\mathrm{~d}, 6 \mathrm{H}, J=7 \mathrm{~Hz}), 0.84(\mathrm{~d}, 6 \mathrm{H}, J=7 \mathrm{~Hz}), 0.69(\mathrm{~d}, 6 \mathrm{H}, J=6.5 \mathrm{~Hz})$ ppm.

${ }^{13} \mathrm{C}$ NMR $\left(\mathrm{C}_{6} \mathrm{D}_{6}, 125 \mathrm{MHz}, 298 \mathrm{~K}\right): \delta 218.2,160.4,146.6,145.3,144.8,140.9,136.6,133.1$, $132.3,131.6,131.1,129.9,129.6,128.5,128.4,127.9,127.5,125.5,125.2$, 124.8, 29.2, 28.8, 24.4, 24.3, 23.8, 23.5 ppm.

Elemental analysis: Anal. Calcd for $\mathrm{C}_{43} \mathrm{H}_{44} \mathrm{FeN}_{2} \mathrm{O}_{4}$ : C, 72.88; H, 6.26; N, 3.95. Found: C, 72.72; H, 7.01; N, 3.90. IR: (Film, $v_{\mathrm{CO}} \mathrm{cm}^{-1}$ ): 1998, 1912, 1871.

IR: (Film, $\left.v_{\mathrm{CO}} \mathrm{cm}^{-1}\right):$ 1998, 1912, 1871.

\section{Synthesis of ( $a$ NHC)iron-tricarbonyl silyl hydride complex :}

\section{Synthesis of the complex $9 ;(a \mathrm{NHC}) \mathrm{Fe}(\mathrm{H})\left(\mathrm{SiH}_{2} \mathrm{Ph}\right)(\mathrm{CO})_{3}$ :}

In a glovebox, an oven dried $25 \mathrm{~mL}$ Schlenk flask was charged with complex 1; $(a \mathrm{NHC}) \mathrm{Fe}(\mathrm{CO})_{4}$ (100 mg, $0.141 \mathrm{mmol})$ in $5 \mathrm{~mL}$ benzene. Then $\mathrm{PhSiH}_{3}(122.0 \mu \mathrm{L}, 0.987 \mathrm{mmol})$ was added and the reaction mixture was heated to $85{ }^{\circ} \mathrm{C}$ for $24 \mathrm{~h}$. After completion of the reaction, pale yellow colored solution was separated from the reaction mixture simply by decantation. Then the supernatant was concentrated to dryness and two times washing with cold hexane afforded pale yellow color complex 9 (35.4 mg, $0.0449 \mathrm{mmol}, 32 \%)$.

${ }^{1} \mathrm{H}$ NMR $\left(\mathrm{C}_{6} \mathrm{D}_{6}, 500 \mathrm{MHz}, 298 \mathrm{~K}\right): \delta 8.21(\mathrm{~d}, 2 \mathrm{H}, J=8.3 \mathrm{~Hz}), 7.24$ (t, 3H, J = 7.4 Hz), 7.10-7.19 (m, 5H), 7.02-7.06(m, 2H), $6.95(\mathrm{~d}, 2 \mathrm{H}, J=7.3 \mathrm{~Hz}), 6.83(\mathrm{t}, 4 \mathrm{H}, J=7.3 \mathrm{~Hz}), 6.64(\mathrm{t}, 1 \mathrm{H}, J=8.2$ $\mathrm{Hz}), 6.55(\mathrm{t}, 2 \mathrm{H}, J=8.3 \mathrm{~Hz}), 5.36(\mathrm{~s}, 2 \mathrm{H}), 2.35-2.43(\mathrm{~m}, 4 \mathrm{H}), 1.16(\mathrm{~d}, 6 \mathrm{H}, J=6.4 \mathrm{~Hz}), 0.81$ (d, $6 \mathrm{H}, J=6.4 \mathrm{~Hz}), 0.69(\mathrm{t}, 12 \mathrm{H}, J=7.4 \mathrm{~Hz}),-9.22(\mathrm{~s}, 1 \mathrm{H}) \mathrm{ppm}$.

${ }^{13} \mathrm{C}$ NMR $\left(\mathrm{C}_{6} \mathrm{D}_{6}, 125 \mathrm{MHz}, 298 \mathrm{~K}\right): \delta 218.2,155.7,145.4,144.7,136.1,132.9,132.8,132.7$, $131.3,130.1,129.8,129.7,129.2,128.9,128.5,128.4,128.2,128.0,127.3,126.9,126.2,125.7$, $122.7,120.9,29.6,29.3,25.4,23.8,23.4,22.7 \mathrm{ppm}$. 
${ }^{29} \mathrm{Si}\left\{{ }^{1} \mathrm{H}\right\}$ NMR $\left(99.36 \mathrm{MHz}, \mathrm{C}_{6} \mathrm{D}_{6}\right): \delta-6.07 \mathrm{ppm}$.

IR: (Film, $\left.v_{\mathrm{CO}} \mathrm{cm}^{-1}\right)$ : 2007, 1963, 1893.

\section{Synthesis of the complex 11; $(a \mathrm{NHC}) \mathrm{Fe}\left\{(p\right.$-anisyl $) \mathrm{CH}_{2} \mathrm{~N}(p$-tolyl $\left.)\right\}\left(\mathrm{SiH}_{2} \mathrm{Ph}\right)(\mathrm{CO})_{3}$ :}

A Screw cap NMR tube was charged with $(a \mathrm{NHC}) \mathrm{Fe}(\mathrm{H})\left(\mathrm{SiH}_{2} \mathrm{Ph}\right)(\mathrm{CO})_{3}(15 \mathrm{mg}, 0.019 \mathrm{mmol})$ and $\{($ para-anisyl $) \mathrm{CH}=\mathrm{N}$ (para-tolyl $)\}(4.3 \mathrm{mg}, 0.019 \mathrm{mmol})$ and $0.6 \mathrm{~mL}$ DMSO-d $\mathrm{d}_{6}$ were added to the reaction mixture. Then the reaction mixture was heated to $75{ }^{\circ} \mathrm{Cand}$ the reaction was monitored by ${ }^{1} \mathrm{H}$ NMR. After $6 \mathrm{~h}$, the conversion to the desired compound11 was found to be nearly complete by ${ }^{1} \mathrm{H}$ NMR spectroscopy.

${ }^{1} \mathrm{H}$ NMR (DMSO-d 6 , $\left.500 \mathrm{MHz}, 298 \mathrm{~K}\right): \delta 9.08(\mathrm{~s}, 1 \mathrm{H}), 7.68(\mathrm{t}, 2 \mathrm{H}, \mathrm{J}=8.0 \mathrm{~Hz}), 7.63(\mathrm{t}, 2 \mathrm{H}, \mathrm{J}=$ $7.5 \mathrm{~Hz}), 7.48(\mathrm{~d}, 6 \mathrm{H}, \mathrm{J}=8.0 \mathrm{~Hz}), 7.38-7.45(\mathrm{~m}, 3 \mathrm{H}), 7.24-7.26(\mathrm{~m}, 7 \mathrm{H}), 6.92(\mathrm{~d}, 2 \mathrm{H}, \mathrm{J}=7.5 \mathrm{~Hz})$, 6.82-6.87 (m, 4H), $6.46(\mathrm{~d}, 2 \mathrm{H}, \mathrm{J}=8.5 \mathrm{~Hz}), 5.74(\mathrm{~s}, 2 \mathrm{H}), 4.13(\mathrm{~d}, 2 \mathrm{H}, \mathrm{J}=5.5 \mathrm{~Hz}), 3.71(\mathrm{~s}, 3 \mathrm{H})$, $2.11(\mathrm{~s}, 3 \mathrm{H}), 1.26(\mathrm{~d}, 6 \mathrm{H}, \mathrm{J}=6.5 \mathrm{~Hz}), 1.04(\mathrm{~d}, 6 \mathrm{H}, \mathrm{J}=6.5 \mathrm{~Hz}), 0.82(\mathrm{~d}, 12 \mathrm{H}, \mathrm{J}=6.5 \mathrm{~Hz}) \mathrm{ppm}$.

${ }^{13} \mathrm{C}$ NMR (DMSO-d $6,125 \mathrm{MHz}, 298 \mathrm{~K}$ ): $\delta$ 220.0, 191.1, 146.3, 145.1, 144.5, 144.2, 136.0, 133.9, 132.8, 132.6, 132.0, 131.6, 130.5, 129.9, 129.2, 129.1, 129.0, 128.2, 127.9, 127.8, 127.6, $127.4,126.0,125.1,124.6,123.7,120.1,113.5,112.3,54.8,46.0,28.7,28.4,25.0,23.4,22.8$, $21.9,19.9 \mathrm{ppm}$. 
Table S1. Optimisation study for the reduction of N-benzylideneaniline with $(a \mathrm{NHC})$ iron complex1 $^{[\mathrm{ad}]}$ :
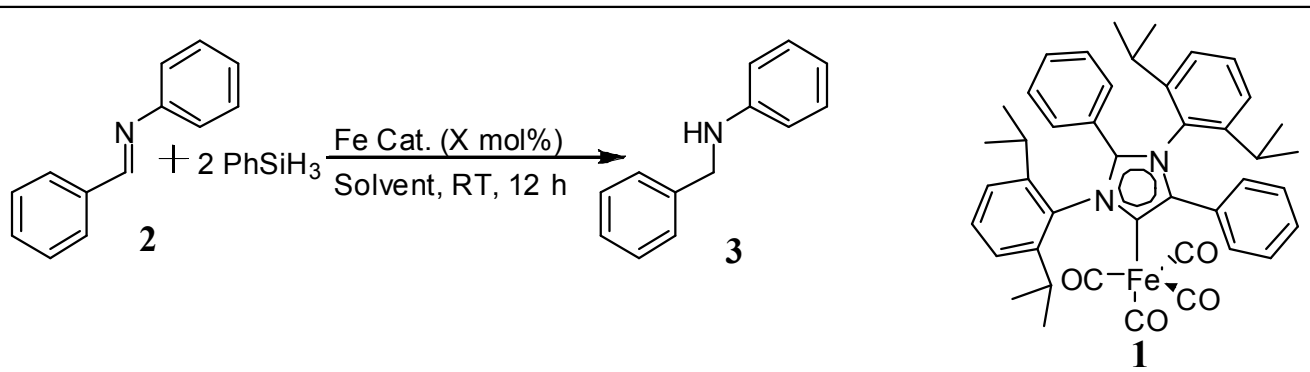

\begin{tabular}{|c|c|c|c|c|c|c|}
\hline Entry & Silane & Solvent & Cat./ mol\% & Time/h & Conversion $(\%)^{[b]}$ & TON \\
\hline 1 & $\mathrm{PhSiH}_{3}$ & $\mathrm{EtOH}$ & 5 & 6 & 95 & \\
\hline 2 & $\mathrm{PhSiH}_{3}$ & DMSO & 5 & 6 & $>99$ & \\
\hline 3 & $\mathrm{PhSiH}_{3}$ & $\mathrm{Et}_{2} \mathrm{O}$ & 5 & 6 & 20 & \\
\hline 4 & $\mathrm{PhSiH}_{3}$ & THF & 5 & 6 & 25 & \\
\hline 5 & $\mathrm{PhSiH}_{3}$ & DCM & 5 & 6 & 45 & \\
\hline 6 & $\mathrm{PhSiH}_{3}$ & $\mathrm{H}_{2} \mathrm{O}$ & 5 & 24 & - & \\
\hline 7 & $\mathrm{PhSiH}_{3}$ & DMSO & 5 & 6 & $56^{[c]}$ & \\
\hline 8 & PMHS & DMSO & 5 & 6 & 25 & \\
\hline 9 & $\mathrm{Ph}_{2} \mathrm{SiH}_{2}$ & DMSO & 2 & 6 & 35 & \\
\hline 10 & $\mathrm{Ph}_{3} \mathrm{SiH}$ & DMSO & 2 & 6 & - & \\
\hline 11 & $\mathrm{PhSiH}_{3}$ & DMSO & 2 & 6 & $>99$ & \\
\hline 12 & $\mathrm{PhSiH}_{3}$ & DMSO & 1 & 6 & 96 & \\
\hline 13 & $\mathrm{PhSiH}_{3}$ & DMSO & 1 & 10 & $>99$ & \\
\hline 14 & $\mathrm{PhSiH}_{3}$ & DMSO & 0.5 & 10 & $>99$ & 200 \\
\hline 15 & $\mathrm{PhSiH}_{3}$ & DMSO & 0.1 & 10 & $>99$ & 1,000 \\
\hline 16 & $\mathrm{PhSiH}_{3}$ & DMSO & 0.05 & 12 & $>99$ & 2,000 \\
\hline 17 & PMHS (5 eqv.) & DMSO & 0.05 & 12 & 48 & \\
\hline 18 & PMHS (10 eqv.) & DMSO & 0.05 & 12 & 63 & \\
\hline 19 & $(\mathrm{TMSO})_{2} \mathrm{SiMeH}$ & DMSO & 0.05 & 12 & 66 & \\
\hline 20 & $\mathrm{PhSiH}_{3}$ & DMSO & 0.01 & 12 & 97 & 9,700 \\
\hline 21 & $\mathrm{PhSiH}_{3}$ & DMSO & 0.005 & 12 & 70 & 14,000 \\
\hline 22 & $\mathrm{PhSiH}_{3}$ & DMSO & 0.005 & 24 & 85 & 17,000 \\
\hline 23 & $\mathrm{PhSiH}_{3}$ & DMSO & - & 48 & - & \\
\hline
\end{tabular}


[a] Aldimine $(0.5 \mathrm{mmol})$, silane $(1 \mathrm{mmol})$, catalyst 1 , DMSO $(1 \mathrm{~mL})$. [b] Determined by ${ }^{1} \mathrm{H}$ NMR. [c] $\mathrm{PhSiH}_{3}$ (0.5 mmol, 1 equiv.).

\section{General procedure for catalytic hydrosilylation of aldimines to secondary amines by $(a \mathrm{NHC}) \mathrm{Fe}(\mathrm{CO})_{4}$ :}

ASchlenk tube was charged with the catalyst $1(0.00025 \mathrm{mmol}, 0.177 \mathrm{mg}, 0.05 \mathrm{~mol} \%)$, aldimine $(0.5 \mathrm{mmol}), \mathrm{PhSiH}_{3}(1.0 \mathrm{mmol}, 123.4 \mu \mathrm{L})$, and DMSO $(1.0 \mathrm{~mL})$ with magnetic stirring bar under air.The reaction mixture was stirred at room temperature for $12 \mathrm{~h}$.After the completion of the reaction, the reaction mixture was worked up with ether $(3 \mathrm{X} 10 \mathrm{~mL})$ and water $(3 \mathrm{X} 15$ $\mathrm{mL}$ ). The combined organicfractions were dried under reduced pressure. The extent of conversion was determined by ${ }^{1} \mathrm{HNMR}$. The amine was purified by using a silica gel chromatography column and amixture of hexane/ethyl acetate $(2-5 \%)$ as the eluent.

\section{Characterization data for secondary amines:}

N-benzylaniline ${ }^{1}$ (Table 1, Entry 1)<smiles>c1ccc(CNc2ccccc2)cc1</smiles>

The compound was prepared as described in the general procedure (Yellow oil, 96\% isolatedyield, $88 \mathrm{mg}$ ).

${ }^{1} \mathrm{H}$ NMR (400 MHz, $\left.\mathrm{CDCl}_{3}, 298 \mathrm{~K}\right): \delta 7.35-7.41(\mathrm{~m}, 4 \mathrm{H}), 7.31(\mathrm{~d}, 1 \mathrm{H}, J=6.1 \mathrm{~Hz}), 7.20(\mathrm{t}, 2 \mathrm{H}, J$ $=8.4 \mathrm{~Hz}), 6.75(\mathrm{t}, 1 \mathrm{H}, J=7.6 \mathrm{~Hz}), 6.67(\mathrm{~d}, 2 \mathrm{H}, J=7.6 \mathrm{~Hz}), 4.36(\mathrm{~s}, 2 \mathrm{H}) \mathrm{ppm}$.

${ }^{13} \mathrm{C}\left\{{ }^{1} \mathrm{H}\right\} \mathrm{NMR}\left(100 \mathrm{MHz}, \mathrm{CDCl}_{3}, 298 \mathrm{~K}\right): \delta$ 148.0, 139.3, 129.2, 128.6, 127.5, 127.2, 117.6, $112.9,48.3 \mathrm{ppm}$.

N-benzyl-4-methylaniline ${ }^{1}$ (Table 1, Entry 2)<smiles>Cc1ccc(NCc2ccccc2)cc1</smiles>

The compound was prepared as described in the general procedure (Light yellow oil, 92\% isolated yield, $90 \mathrm{mg}$ ). 
${ }^{1} \mathrm{H}$ NMR $\left(400 \mathrm{MHz}, \mathrm{CDCl}_{3}, 298 \mathrm{~K}\right): \delta 7.33-7.39(\mathrm{~m}, 4 \mathrm{H}), 7.28(\mathrm{~d}, 1 \mathrm{H}, J=6.9 \mathrm{~Hz}), 7.00(\mathrm{~d}, 2 \mathrm{H}, J$ $=8.4 \mathrm{~Hz}), 6.58(\mathrm{~d}, 2 \mathrm{H}, J=8.4 \mathrm{~Hz}), 4.31(\mathrm{~s}, 2 \mathrm{H}), 2.26(\mathrm{~s}, 3 \mathrm{H}) \mathrm{ppm}$.

${ }^{13} \mathrm{C}\left\{{ }^{1} \mathrm{H}\right\}$ NMR $\left(100 \mathrm{MHz}, \mathrm{CDCl}_{3}, 298 \mathrm{~K}\right): \delta 145.8,139.6,129.7,128.5,127.4,127.1,126.7$, 113.0, 48.6, $20.3 \mathrm{ppm}$.

N-Benzyl-2-methylaniline ${ }^{2}$ (Table 1, Entry 3)<smiles>Cc1ccccc1NCc1ccccc1</smiles>

The compound was prepared as described in the general procedure (White solid, $78 \%$ isolated yield, $77 \mathrm{mg}$ ).

${ }^{1} \mathrm{H}$ NMR (400 MHz, $\left.\mathrm{CDCl}_{3}, 298 \mathrm{~K}\right): \delta$ 7.25-7.39 (m, 5H), 7.07-7.12 (m, 2H), 6.61-6.70 (m, 2H), 4.38 (s, 2H), 3.87 (brs, 1H), 2.17 (s, 3H) ppm.

${ }^{13} \mathrm{C}\left\{{ }^{1} \mathrm{H}\right\}$ NMR (100 MHz, $\left.\mathrm{CDCl}_{3}, 298 \mathrm{~K}\right): \delta 146.0,139.5,130.0,128.6,127.6,127.2,127.1$, $121.9,117.2,109.9,48.3,17.6 \mathrm{ppm}$.

N-(4-Methylbenzyl)-4-methylaniline ${ }^{1}$ (Table 1, Entry 5)

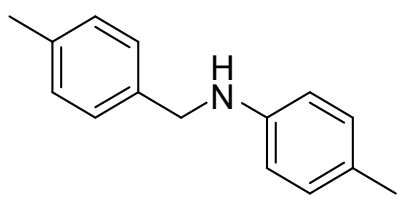

The compound was prepared as described in the general procedure (Yellow solid, 95\% isolated yield, $100 \mathrm{mg}$ ).

${ }^{1} \mathrm{H}$ NMR (400 MHz, $\left.\mathrm{CDCl}_{3}, 298 \mathrm{~K}\right): \delta 7.29$ (d, 2H, $\left.J=7.6 \mathrm{~Hz}\right), 7.18$ (d, 2H, $\left.J=7.6 \mathrm{~Hz}\right), 7.02(\mathrm{~d}$, $2 \mathrm{H}, J=8.4 \mathrm{~Hz}$ ), 6.60 (d, 2H, $J=8.4 \mathrm{~Hz}), 4.29$ (s, 2H), 2.38 (s, 3H), 2.27 (s, 3H) ppm.

${ }^{13} \mathrm{C}\left\{{ }^{1} \mathrm{H}\right\}$ NMR (100 MHz, $\left.\mathrm{CDCl}_{3}, 298 \mathrm{~K}\right): \delta 145.9,136.7,136.5,129.7,129.2,127.5,126.7$, 113.0, 48.4, 21.1, $20.4 \mathrm{ppm}$.

N-(4-Methoxybenzyl)-4-methylaniline ${ }^{1}$ (Table 1, Entry 6)<smiles>COc1ccc(CNc2ccc(C)cc2)cc1</smiles> 
The compound was prepared as described in the general procedure (Light yellow solid, $96 \%$ isolated yield, $\mathrm{m}=109 \mathrm{mg}$ ).

${ }^{1} \mathrm{H}$ NMR (400 MHz, $\left.\mathrm{CDCl}_{3}, 298 \mathrm{~K}\right): \delta 7.31(\mathrm{~d}, 2 \mathrm{H}, J=8.4 \mathrm{~Hz}), 7.02(\mathrm{~d}, 2 \mathrm{H}, J=7.6 \mathrm{~Hz}), 6.91(\mathrm{~d}$, $2 \mathrm{H}, J=7.6 \mathrm{~Hz}), 6.59$ (d, 2H, $J=7.6 \mathrm{~Hz}), 4.26(\mathrm{~s}, 2 \mathrm{H}), 3.82(\mathrm{~s}, 3 \mathrm{H}), 2.27$ (s, 3H) ppm.

${ }^{13} \mathrm{C}\left\{{ }^{1} \mathrm{H}\right\}$ NMR $\left(100 \mathrm{MHz}, \mathrm{CDCl}_{3}, 298 \mathrm{~K}\right): \delta 158.7,145.9,131.6,129.7,128.7,126.6,113.9$, $112.9,55.2,48.0,20.3 \mathrm{ppm}$.

N-(4'-(N',N'-Dimethyl)benzyl)-4-methylaniline ${ }^{1}$ (Table 1, Entry 7)

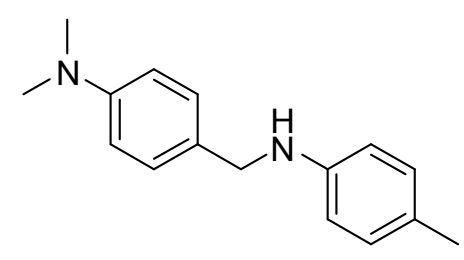

The compound was prepared as described in the general procedure (Yellow solid, 93\% isolated yield, $112 \mathrm{mg}$ ).

${ }^{1} \mathrm{H}$ NMR (400 MHz, $\left.\mathrm{CDCl}_{3}, 298 \mathrm{~K}\right): \delta 7.25$ (d, 2H, $\left.J=8.4 \mathrm{~Hz}\right), 6.99$ (d, 2H, $\left.J=8.4 \mathrm{~Hz}\right), 6.73(\mathrm{~d}$, $2 \mathrm{H}, J=9.1 \mathrm{~Hz}$ ), 6.58 (d, 2H, $J=8.4 \mathrm{~Hz}$ ), 4.19 (s, 2H), 2.94 (s, 3H), 2.25 (s, 3H) ppm.

${ }^{13} \mathrm{C}\left\{{ }^{1} \mathrm{H}\right\}$ NMR (100 MHz, $\left.\mathrm{CDCl}_{3}, 298 \mathrm{~K}\right): \delta 149.9,146.2,129.7,128.7,127.3,126.4,112.9$, $112.7,48.2,40.7,20.4 \mathrm{ppm}$.

N-(4-Iodobenzyl)-4-methylaniline (Table 1, Entry 8)

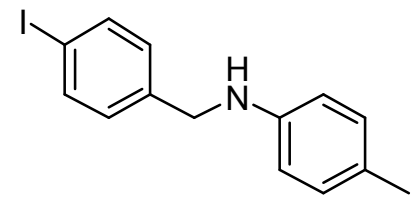

The compound was prepared as described in the general procedure (Light yellow solid, 95\% isolated yield, $153.5 \mathrm{mg}$ ).

${ }^{1} \mathrm{H}$ NMR (400 MHz, $\left.\mathrm{CDCl}_{3}, 298 \mathrm{~K}\right): \delta 7.67(\mathrm{~d}, 2 \mathrm{H}, J=8.4 \mathrm{~Hz}), 7.13(\mathrm{~d}, 2 \mathrm{H}, J=7.6 \mathrm{~Hz}), 7.00(\mathrm{~d}$, $2 \mathrm{H}, J=7.6 \mathrm{~Hz}), 6.54$ (d, 2H, $J=8.4 \mathrm{~Hz}), 4.28(\mathrm{~s}, 2 \mathrm{H}), 2.26(\mathrm{~s}, 3 \mathrm{H}) \mathrm{ppm}$.

${ }^{13} \mathrm{C}\left\{{ }^{1} \mathrm{H}\right\}$ NMR (100 MHz, $\left.\mathrm{CDCl}_{3}, 298 \mathrm{~K}\right): \delta 145.5,139.4,137.5,129.7,129.3,126.9,112.9$, 92.2, 47.9, $20.4 \mathrm{ppm}$.

ESI-MS: $\mathrm{m} / \mathrm{z}$ calc. for $\mathrm{C}_{14} \mathrm{H}_{14} \mathrm{INH}[\mathrm{M}+\mathrm{H}]^{+}$324.0204, found 324.0206 . 
N-(4-Bromobenzyl)-4-methylaniline ${ }^{1}$ (Table 1, Entry 9)<smiles>Cc1ccc(NCc2ccc(Br)cc2)cc1</smiles>

The compound was prepared as described in the general procedure (Light yellow solid, 95\% isolated yield, $131 \mathrm{mg})$.

${ }^{1} \mathrm{H}$ NMR (400 MHz, $\left.\mathrm{CDCl}_{3}, 298 \mathrm{~K}\right): \delta 7.45$ (d, 2H, $\left.J=8.4 \mathrm{~Hz}\right), 7.24$ (d, 2H, $\left.J=7.6 \mathrm{~Hz}\right), 6.98$ (d, $2 \mathrm{H}, J=8.4 \mathrm{~Hz}), 6.53(\mathrm{~d}, 2 \mathrm{H}, J=8.4 \mathrm{~Hz}), 4.27$ (s, 2H), 3.94 (brs, 1H), 2.24 (s, 3H) ppm.

${ }^{13} \mathrm{C}\left\{{ }^{1} \mathrm{H}\right\}$ NMR $\left(100 \mathrm{MHz}, \mathrm{CDCl}_{3}, 298 \mathrm{~K}\right): \delta$ 145.5, 138.7, 131.6, 129.7, 129.0, 127.0, 120.8, 113.0, 49.9, $20.4 \mathrm{ppm}$.

N-(4-Chlorobenzyl)-4-methylaniline ${ }^{1}$ (Table 1, Entry 10)<smiles>Cc1ccc(NCc2ccc(Cl)cc2)cc1</smiles>

The compound was prepared as described in the general procedure (Yellow solid, 94\% isolated yield, $109 \mathrm{mg}$ ).

${ }^{1} \mathrm{H}$ NMR (400 MHz, $\left.\mathrm{CDCl}_{3}, 298 \mathrm{~K}\right): \delta 7.28-7.34(\mathrm{~m}, 4 \mathrm{H}), 6.99(\mathrm{~d}, 2 \mathrm{H}, J=8.4 \mathrm{~Hz}), 6.54(\mathrm{~d}, 2 \mathrm{H}, \mathrm{J}$ $=8.4 \mathrm{~Hz}), 4.29(\mathrm{~s}, 2 \mathrm{H}), 3.95(\mathrm{brs}, 1 \mathrm{H}), 2.25(\mathrm{~s}, 3 \mathrm{H}) \mathrm{ppm}$.

${ }^{13} \mathrm{C}\left\{{ }^{1} \mathrm{H}\right\} \mathrm{NMR}\left(100 \mathrm{MHz}, \mathrm{CDCl}_{3}, 298 \mathrm{~K}\right): \delta 145.5,138.2,132.7,129.7,129.7,128.6,127.0$, 113.0, 47.9, 20.4 ppm.

N-(4-Cyanobenzyl)-4-methylaniline ${ }^{2}$ (Table 1, Entry 11)<smiles>Cc1ccc(NCc2ccc(C#N)cc2)cc1</smiles>

The compound was prepared as described in the general procedure (Yellow solid, 94\% isolated yield,106 mg).

${ }^{1} \mathrm{H}$ NMR $\left(500 \mathrm{MHz}, \mathrm{CDCl}_{3}, 298 \mathrm{~K}\right): \delta 7.61(\mathrm{~d}, 2 \mathrm{H}, J=8.0 \mathrm{~Hz}), 7.47(\mathrm{~d}, 2 \mathrm{H}, J=8.5 \mathrm{~Hz}), 6.98$ $(\mathrm{d}, 2 \mathrm{H}, J=8.0 \mathrm{~Hz}), 6.50(\mathrm{~d}, 2 \mathrm{H}, J=8.5 \mathrm{~Hz}), 4.41$ (s, 2H), 2.24 (s, 3H) ppm. 
${ }^{13} \mathrm{C}\left\{{ }^{1} \mathrm{H}\right\}$ NMR (125 MHz, $\left.\mathrm{CDCl}_{3}, 298 \mathrm{~K}\right): \delta$ 145.6, 145.1, 132.4, 129.8, 127.7, 127.3, 118.9, $113.0,110.8,48.1,20.3 \mathrm{ppm}$.

4-Methyl-N-(4-nitrobenzyl)aniline ${ }^{2}$ (Table 1, Entry 12)<smiles>Cc1ccc(NCc2ccc([N+](=O)[O-])cc2)cc1</smiles>

The compound was prepared as described in the general procedure (Yellow solid, 94\% isolated yield, $103 \mathrm{mg}$ ).

${ }^{1} \mathrm{H}$ NMR (400 MHz, $\left.\mathrm{CDCl}_{3}, 298 \mathrm{~K}\right): \delta 8.18(\mathrm{~d}, 2 \mathrm{H}, J=8.5 \mathrm{~Hz}), 7.53(\mathrm{~d}, 2 \mathrm{H}, J=8.5 \mathrm{~Hz}), 6.98$ (d, $2 \mathrm{H}, J=7.9 \mathrm{~Hz}), 6.50$ (d, 2H, $J=7.9 \mathrm{~Hz}), 4.45$ (s, 2H), 2.23 (s, 3H) ppm.

${ }^{13} \mathrm{C}\left\{{ }^{1} \mathrm{H}\right\}$ NMR $\left(100 \mathrm{MHz}, \mathrm{CDCl}_{3}, 298 \mathrm{~K}\right): \delta 147.7,147.1,145.0,129.8,127.7,127.4,123.8$, 113.0, 47.9, $20.3 \mathrm{ppm}$.

N-[4-(Methyloxycarbonyl)benzyl]-4-methylaniline ${ }^{1}$ (Table 1, Entry 14)<smiles>COC(=O)c1ccc(CNc2ccc(C)cc2)cc1</smiles>

The compound was prepared as described in the general procedure (Yellow solid, $94 \%$ isolated yield, $108 \mathrm{mg}$ ).

${ }^{1} \mathrm{H}$ NMR (400 MHz, $\left.\mathrm{CDCl}_{3}, 298 \mathrm{~K}\right): \delta 7.98(\mathrm{~d}, 2 \mathrm{H}, J=8.5 \mathrm{~Hz}), 7.43(\mathrm{~d}, 2 \mathrm{H}, J=8.5 \mathrm{~Hz}$ ), 6.99 (d, $2 \mathrm{H}, J=8.0 \mathrm{~Hz}), 6.62(\mathrm{~d}, 2 \mathrm{H}, J=8.0 \mathrm{~Hz}), 4.38$ (s, 2H), 3.90 (s, 3H), 2.24 (s, 3H) ppm.

${ }^{13} \mathrm{C}\left\{{ }^{1} \mathrm{H}\right\}$ NMR $\left(100 \mathrm{MHz}, \mathrm{CDCl}_{3}, 298 \mathrm{~K}\right): \delta 166.9,143.8,143.7,129.9,129.8,129.3,127.6$, $126.9,114.4,52.0,49.2,20.4 \mathrm{ppm}$.

N-[(Ferrocenyl)methyl]4-methylaniline ${ }^{1}$ (Table 1, Entry 15)

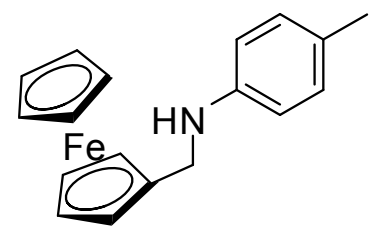

The compound was prepared as described in the general procedure (Yellow solid, 95\% isolated yield, $145.0 \mathrm{mg}$ ). 
${ }^{1} \mathrm{H}$ NMR (400 MHz, $\left.\mathrm{CDCl}_{3}, 298 \mathrm{~K}\right): \delta 7.03(\mathrm{~d}, 2 \mathrm{H}, J=7.9 \mathrm{~Hz}), 6.61(\mathrm{~d}, 2 \mathrm{H}, J=8.5 \mathrm{~Hz}), 4.26(\mathrm{t}$, $2 \mathrm{H}, J=1.8 \mathrm{~Hz}), 4.19(\mathrm{~s}, 5 \mathrm{H}), 4.15(\mathrm{t}, 2 \mathrm{H}, J=1.8 \mathrm{~Hz}), 3.95(\mathrm{~s}, 2 \mathrm{H}), 2.27$ (s, 3H) ppm.

${ }^{13} \mathrm{C}\left\{{ }^{1} \mathrm{H}\right\} \mathrm{NMR}\left(100 \mathrm{MHz}, \mathrm{CDCl}_{3}, 298 \mathrm{~K}\right): \delta 146.1,129.7,126.7,113.0,86.7,68.5,68.1,67.8$, 43.7, $20.4 \mathrm{ppm}$.

N-(4-Cyanobenzyl)-4-methoxylaniline ${ }^{1}$ (Table 1, Entry 16)<smiles>COc1ccc(NCc2ccc(C#N)cc2)cc1</smiles>

The compound was prepared as described in the general procedure (Orange solid, 92\% isolated yield, $109 \mathrm{mg})$.

${ }^{1} \mathrm{H}$ NMR (500 MHz, $\left.\mathrm{CDCl}_{3}, 298 \mathrm{~K}\right): \delta 7.61(\mathrm{~d}, 2 \mathrm{H}, J=8.0 \mathrm{~Hz}), 7.47(\mathrm{~d}, 2 \mathrm{H}, J=8.0 \mathrm{~Hz}), 6.76$ $(\mathrm{d}, 2 \mathrm{H}, J=9.0 \mathrm{~Hz}), 6.54(\mathrm{~d}, 2 \mathrm{H}, J=9.0 \mathrm{~Hz}), 4.37$ (s, 2H), 3.73(s, 3H) ppm.

${ }^{13} \mathrm{C}\left\{{ }^{1} \mathrm{H}\right\}$ NMR $\left(125 \mathrm{MHz}, \mathrm{CDCl}_{3}, 298 \mathrm{~K}\right): \delta 152.5,145.6,141.5,132.4,127.7,118.8,114.9$, $114.2,110.8,55.7,48.7 \mathrm{ppm}$.

N-(4-nitrobenzyl)-4-Methylaniline ${ }^{1}$ (Table 1, Entry 17)

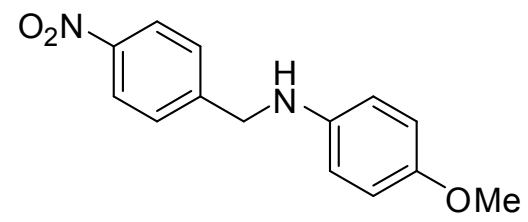

The compound was prepared as described in the general procedure (Orange solid, $80 \%$ isolated yield, $103 \mathrm{mg})$.

${ }^{1} \mathrm{H}$ NMR (500 MHz, $\left.\mathrm{CDCl}_{3}, 298 \mathrm{~K}\right): \delta 8.18(\mathrm{~d}, 2 \mathrm{H}, J=8.5 \mathrm{~Hz}), 7.53(\mathrm{~d}, 2 \mathrm{H}, J=8.5 \mathrm{~Hz}), 6.76$ $(\mathrm{d}, 2 \mathrm{H}, J=9.0 \mathrm{~Hz}), 6.54(\mathrm{~d}, 2 \mathrm{H}, J=9.0 \mathrm{~Hz}), 4.42$ (s, 2H), 3.73 (s, 3H) ppm.

${ }^{13} \mathrm{C}\left\{{ }^{1} \mathrm{H}\right\}$ NMR $\left(125 \mathrm{MHz}, \mathrm{CDCl}_{3}, 298 \mathrm{~K}\right): \delta 152.5,147.7,141.4,127.7,123.8,116.4,114.9$, 114.2, 55.7, $48.5 \mathrm{ppm}$.

4-Methoxy-N-(3,4,5-trimethoxybenzyl)aniline ${ }^{1}$ (Table 1, Entry 18)<smiles>COc1ccc(NCc2cc(OC)c(OC)c(OC)c2)cc1</smiles> 
The compound was prepared as described in the general procedure (Yellow solid, 94\% isolated yield, $145 \mathrm{mg})$.

${ }^{1} \mathrm{H}$ NMR (400 MHz, $\left.\mathrm{CDCl}_{3}, 298 \mathrm{~K}\right): \delta 6.78$ (d, 2H, $\left.J=8.6 \mathrm{~Hz}\right), 6.60-6.62(\mathrm{~m}, 4 \mathrm{H}), 4.21(\mathrm{~s}, 2 \mathrm{H})$, $3.84(\mathrm{~s}, 9 \mathrm{H}), 3.74(\mathrm{~s}, 3 \mathrm{H}) \mathrm{ppm}$.

${ }^{13} \mathrm{C}\left\{{ }^{1} \mathrm{H}\right\}$ NMR $\left(100 \mathrm{MHz}, \mathrm{CDCl}_{3}, 298 \mathrm{~K}\right): \delta 153.3,152.2,142.4,136.8,135.4,114.8,114.1$, $104.2,60.8,56.0,55.7,49.6 \mathrm{ppm}$.

2-\{(phenylamino)methyl $\}$ phenol (Table 1, Entry 19)<smiles>Oc1ccccc1CNc1ccccc1</smiles>

The compound was prepared as described in the general procedure (Brown solid, 91\% isolated yield, $90 \mathrm{mg}$ ).

${ }^{1} \mathrm{H}$ NMR (500 MHz, $\left.\mathrm{CDCl}_{3}, 298 \mathrm{~K}\right): \delta 7.30(\mathrm{~d}, 1 \mathrm{H}, J=1.5 \mathrm{~Hz}), 7.19-7.25(\mathrm{~m}, 4 \mathrm{H}), 7.10(\mathrm{~d}, 1 \mathrm{H}, J$ $=7.5 \mathrm{~Hz}), 6.96-7.00(\mathrm{~m}, 2 \mathrm{H}), 6.90(\mathrm{~d}, 2 \mathrm{H}, J=8.0 \mathrm{~Hz}), 6.84(\mathrm{t}, 1 \mathrm{H}, J=7.0 \mathrm{~Hz}), 4.40(\mathrm{~s}, 2 \mathrm{H})$ ppm.

${ }^{13} \mathrm{C}\left\{{ }^{1} \mathrm{H}\right\}$ NMR $\left(125 \mathrm{MHz}, \mathrm{CDCl}_{3}, 298 \mathrm{~K}\right): \delta 156.6,129.5,129.4,129.2,126.9,122.2,121.2$, 120.0, 117.2, 116.6, 49.5 ppm.

ESI-MS: $\mathrm{m} / \mathrm{z}$ calc. for $\mathrm{C}_{13} \mathrm{H}_{13} \mathrm{NOH}[\mathrm{M}+\mathrm{H}]^{+}$200.1031, found 200.1032.

4-(2-hydroxybenzylaino)benzoic acid (Table 1, Entry 20)

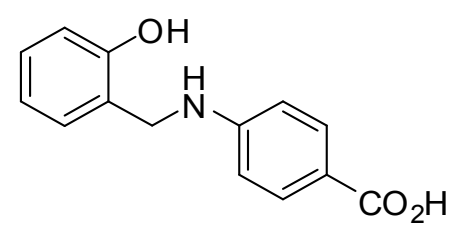

The compound was prepared as described in the general procedure (Light yellow solid, 85\% isolated yield, $103 \mathrm{mg}$ ).

${ }^{1} \mathrm{H}$ NMR (500 MHz, $\mathrm{CDCl}_{3}, 298 \mathrm{~K}$ ): $\delta 12.0$ (brs, 1H), 9.57 (brs, 1H), 7.64 (d, 2H, J=9.0 Hz), $7.14(\mathrm{dd}, 1 \mathrm{H}, J=7.5 \mathrm{~Hz}), 7.05(\mathrm{dt}, 1 \mathrm{H}, J=8.0 \mathrm{~Hz}), 6.82(\mathrm{dd}, 2 \mathrm{H}, J=8.0 \mathrm{~Hz}), 6.72(\mathrm{dt}, 1 \mathrm{H}, J=$ $7.5 \mathrm{~Hz}), 6.58(\mathrm{~d}, 2 \mathrm{H}, J=9.0 \mathrm{~Hz}), 4.24(\mathrm{~d}, 2 \mathrm{H}, J=5.5 \mathrm{~Hz}) \mathrm{ppm}$.

${ }^{13} \mathrm{C}\left\{{ }^{1} \mathrm{H}\right\}$ NMR $\left(125 \mathrm{MHz}, \mathrm{CDCl}_{3}, 298 \mathrm{~K}\right): \delta 167.5,155.0,152.6,131.0,128.2,127.7,124.9$, $118.8,116.9,114.9,110.9,40.8$ ppm.

ESI-MS: m/z calc. for $\mathrm{C}_{14} \mathrm{H}_{13} \mathrm{NO}_{3} \mathrm{Na}[\mathrm{M}+\mathrm{Na}]^{+}$266.0793, found 266.0792. 
N-Cinnamyl-4-methylaniline ${ }^{1}$ (Table 1 , Entry 21$)$

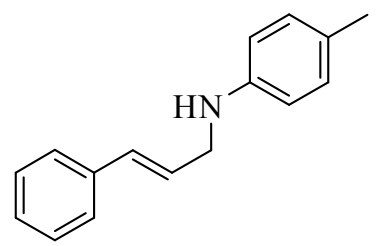

The compound was prepared as described in the general procedure (Yellow oil, 77\% isolated yield, $85 \mathrm{mg}$ ).

${ }^{1} \mathrm{H}$ NMR (500 MHz, $\left.\mathrm{CDCl}_{3}, 298 \mathrm{~K}\right): \delta 7.37-7.53(\mathrm{~m}, 5 \mathrm{H}), 7.16(\mathrm{~d}, 2 \mathrm{H}, \mathrm{J}=8.0 \mathrm{~Hz}), 6.75-6.78(\mathrm{~m}$, $3 \mathrm{H}), 6.48(\mathrm{dt}, 1 \mathrm{H}, \mathrm{J}=6.0 \mathrm{~Hz}, 16 \mathrm{~Hz}), 4.06(\mathrm{dd}, 2 \mathrm{H}, \mathrm{J}=6 \mathrm{~Hz}, 4.5 \mathrm{~Hz}), 3.63$ (q, 1H, J = $7 \mathrm{~Hz}$ ), 2.41 (s, 3H)ppm.

${ }^{13} \mathrm{C}\left\{{ }^{1} \mathrm{H}\right\}$ NMR $\left(125 \mathrm{MHz}, \mathrm{CDCl}_{3}, 298 \mathrm{~K}\right): \delta 145.7,136.9,131.4,129.7,128.5,127.4,127.2$, 126.9, 126.3, 113.3, 46.6, 20.4 ppm.

4-Methoxy-N-(pyridin-2-ylmethyl)aniline ${ }^{1}$ (Table 1, Entry 22)<smiles>COc1ccc(NCc2ccccn2)cc1</smiles>

The compound was prepared as described in the general procedure (Brown solid, 93\% isolated yield, $99 \mathrm{mg}$ ).

${ }^{1} \mathrm{H}$ NMR (400 MHz, $\left.\mathrm{CDCl}_{3}, 298 \mathrm{~K}\right): \delta 8.58(\mathrm{~d}, 1 \mathrm{H}, J=4.3 \mathrm{~Hz}), 7.64(\mathrm{dt}, 1 \mathrm{H}, \mathrm{J}=7.3 \mathrm{~Hz}), 7.34$ $(\mathrm{d}, 1 \mathrm{H}, \mathrm{J}=8.0 \mathrm{~Hz}), 7.18(\mathrm{t}, 1 \mathrm{H}, J=4.9 \mathrm{~Hz}), 6.78(\mathrm{~d}, 2 \mathrm{H}, J=9.2 \mathrm{~Hz}), 6.63(\mathrm{~d}, 2 \mathrm{H}, J=9.2 \mathrm{~Hz})$, $4.42(\mathrm{~s}, 2 \mathrm{H}), 3.74(\mathrm{~s}, 3 \mathrm{H}) \mathrm{ppm}$.

${ }^{13} \mathrm{C}\left\{{ }^{1} \mathrm{H}\right\}$ NMR $\left(100 \mathrm{MHz}, \mathrm{CDCl}_{3}, 298 \mathrm{~K}\right): \delta 158.8,152.2,149.2,142.1,136.6,122.0,121.6$, 114.8, 114.3, 55.7, $50.2 \mathrm{ppm}$.

N-(Anthracen-9-ylmethyl)-4-chloroaniline (Table 1, Entry 24)<smiles>Clc1ccc(NCc2c3ccccc3cc3ccccc23)cc1</smiles>

The compound was prepared as described in the general procedure (Pale yellow solid, 95\% isolated yield, $151 \mathrm{mg}$ ). 
${ }^{1} \mathrm{H}$ NMR $\left(400 \mathrm{MHz}, \mathrm{CDCl}_{3}, 298 \mathrm{~K}\right): \delta 8.49(\mathrm{~s}, 1 \mathrm{H}), 8.24(\mathrm{~d}, 2 \mathrm{H}, J=9.2 \mathrm{~Hz}), 8.05(\mathrm{~d}, 2 \mathrm{H}, J=8.0$ $\mathrm{Hz}), 7.48-7.55(\mathrm{~m}, 4 \mathrm{H}), 7.23(\mathrm{~d}, 2 \mathrm{H}, J=8.6 \mathrm{~Hz}), 6.72(\mathrm{~d}, 2 \mathrm{H}, J=9.2 \mathrm{~Hz}), 5.12(\mathrm{~d}, 2 \mathrm{H}, J=4.3$ $\mathrm{Hz}$ ), 3.83 (brs, 1H) ppm.

${ }^{13} \mathrm{C}\left\{{ }^{1} \mathrm{H}\right\}$ NMR $\left(100 \mathrm{MHz}, \mathrm{CDCl}_{3}, 298 \mathrm{~K}\right): \delta 146.9,131.5,130.4,129.2,129.1,128.9,128.1$, $126.6,125.2,124.0,122.3,113.6,40.9 \mathrm{ppm}$.

ESI-MS: $\mathrm{m} / \mathrm{z}$ calc. for $\mathrm{C}_{21} \mathrm{H}_{16} \mathrm{ClNH}[\mathrm{M}+\mathrm{H}]^{+}$319.0942, found 319.0940 .

Table S2. Optimisation study for the reduction of 4-methyl-N-(1-phenylethylidene)aniline with $\left(a\right.$ NHC)iron complex $1^{\text {a }}$

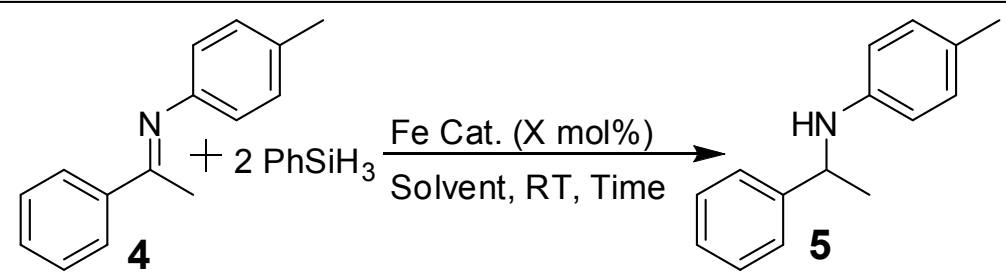

\begin{tabular}{llllll} 
Entry & Silane & Solvent & Cat./ mol\% & Time/h & Conversion $(\%)^{b}$ \\
\hline 1 & $\mathrm{PhSiH}_{3}$ & Toluene & 5 & 24 & 7 \\
2 & $\mathrm{PhSiH}_{3}$ & EtOH & 5 & 24 & $>99$ \\
3 & $\mathrm{PhSiH}_{3}$ & DMSO & 5 & 24 & 31 \\
4 & $\mathrm{PhSiH}_{3}$ & THF & 5 & 24 & \\
5 & $\mathrm{PhSiH}_{3}$ & DMSO & 1 & 24 & 25 \\
6 & $\mathrm{PhSiH}_{3}$ & Toluene & 1 & 24 & 24 \\
7 & $\mathrm{PhSiH}_{3}$ & EtOH & 5 & 12 & 99 \\
8 & $\mathrm{PhSiH}_{3}$ & EtOH & 2 & 12 & 86 \\
9 & $\mathrm{PhSiH}_{3}$ & $\mathrm{EtOH}$ & 2 & 24 & $>99$ \\
10 & $\mathrm{PhSiH}_{3}$ & EtOH & 1 & 24 & 80 \\
11 & $\mathrm{PhSiH}_{3}$ & EtOH & 0.5 & 24 & 55 \\
\hline
\end{tabular}

[a] Ketimine (0.5 mmol), silane (1 mmol), catalyst 1 , EtOH (1 mL). [b] Determined by ${ }^{1} \mathrm{H}$ NMR spectroscopy. 


\section{General procedure for catalytic hydrosilylation of ketimines to secondary amine by $(a \mathrm{NHC}) \mathrm{Fe}(\mathrm{CO})_{4}$ :}

ASchlenk tube was charged with the catalyst $1(0.01 \mathrm{mmol}, 7.09 \mathrm{mg}, 2.0 \mathrm{~mol} \%)$, ketimine reagents $(0.5 \mathrm{mmol}), \mathrm{PhSiH}_{3}(1.0 \mathrm{mmol}, 123.4 \mu \mathrm{L})$, and HPLC-grade ethanol $(1.0 \mathrm{~mL})$ with magnetic stirring bar under air. The reaction mixture was stirred at room temperature for $24 \mathrm{~h}$. After the completion of the reaction, the reaction mixture was worked up with ether $(3 \mathrm{X} 10 \mathrm{~mL})$ and water( 3 X $10 \mathrm{~mL})$. The combined organic fractions were dried under reduced pressure. The extent of conversion was determined by ${ }^{1} \mathrm{H}$ NMR. The amine was purified by using a silica gel chromatography column and a mixture of hexane/ethyl acetate(2-5\%) as the eluent.

\section{Characterization data for secondary amines:}

4-Methyl-N-(1-phenylethyl)aniline ${ }^{1}$ (Table 2, Entry 1)<smiles>Cc1ccc(NC(C)c2ccccc2)cc1</smiles>

The compound was prepared as described in the general procedure (Yellow solid, 95\% isolatedyield, $100 \mathrm{mg}$ ).

${ }^{1} \mathrm{H}$ NMR $\left(400 \mathrm{MHz}, \mathrm{CDCl}_{3}, 298 \mathrm{~K}\right): \delta 7.28-7.44(\mathrm{~m}, 5 \mathrm{H}), 6.96(\mathrm{~d}, 2 \mathrm{H}, J=7.9 \mathrm{~Hz}), 6.50(\mathrm{~d}, 2 \mathrm{H}, J$ $=8.6 \mathrm{~Hz}), 4.52(\mathrm{q}, 1 \mathrm{H}, \mathrm{J}=6.7 \mathrm{~Hz}), 3.96(\mathrm{brs}, 1 \mathrm{H}), 2.25(\mathrm{~s}, 3 \mathrm{H}), 1.56(\mathrm{~d}, 3 \mathrm{H}, J=6.7 \mathrm{~Hz}) \mathrm{ppm}$.

${ }^{13} \mathrm{C}\left\{{ }^{1} \mathrm{H}\right\} \mathrm{NMR}\left(100 \mathrm{MHz}, \mathrm{CDCl}_{3}, 298 \mathrm{~K}\right): \delta 145.4,145.0,129.6,128.6,126.8,126.3,125.8$, $113.4,53.7,25.0,20.3 \mathrm{ppm}$.

4-Methyl-N-[1-(4-methylphenyl)-ethyl] aniline $^{1}$ (Table 2, Entry 2)

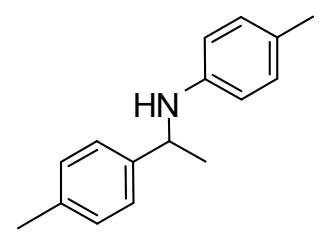

The compound was prepared as described in the general procedure (Yellow oil, 89\% isolated yield, $100 \mathrm{mg})$.

${ }^{1} \mathrm{H}$ NMR $\left(400 \mathrm{MHz}, \mathrm{CDCl}_{3}, 298 \mathrm{~K}\right): \delta 7.28(\mathrm{~d}, 2 \mathrm{H}, J=8.0 \mathrm{~Hz}), 7.15(\mathrm{~d}, 2 \mathrm{H}, J=8.0 \mathrm{~Hz}), 6.93(\mathrm{~d}$, $2 \mathrm{H}, J=7.9 \mathrm{~Hz}), 6.47(\mathrm{~d}, 2 \mathrm{H}, J=8.6 \mathrm{~Hz}), 4.46(\mathrm{q}, 1 \mathrm{H}, \mathrm{J}=6.7 \mathrm{~Hz}), 2.35(\mathrm{~s}, 3 \mathrm{H}), 2.22(\mathrm{~s}, 3 \mathrm{H})$, $1.51(\mathrm{~d}, 3 \mathrm{H}, \mathrm{J}=6.7 \mathrm{~Hz}) \mathrm{ppm}$. 
${ }^{13} \mathrm{C}\left\{{ }^{1} \mathrm{H}\right\}$ NMR (100 MHz, $\left.\mathrm{CDCl}_{3}, 298 \mathrm{~K}\right): \delta 145.0,142.3,136.3,129.5,129.2,126.2,125.7$, $113.4,53.3,25.0,21.0,20.3 \mathrm{ppm}$.

N-\{1-[4-(Trifluoromethyl)phenyl]ethyl\}-4-methylaniline ${ }^{2}$ (Table 2, Entry 4)<smiles>Cc1ccc(NC(C)c2ccc(C(F)(F)F)cc2)cc1</smiles>

The compound was prepared as described in the general procedure (Light yellow oil, $87 \%$ isolated yield, $121 \mathrm{mg}$ ).

${ }^{1} \mathrm{H}$ NMR (500 MHz, $\left.\mathrm{CDCl}_{3}, 298 \mathrm{~K}\right): \delta 7.58(\mathrm{~d}, 2 \mathrm{H}, J=8.0 \mathrm{~Hz}), 7.50(\mathrm{~d}, 2 \mathrm{H}, J=8.0 \mathrm{~Hz}), 6.93(\mathrm{~d}$, $2 \mathrm{H}, J=8.5 \mathrm{~Hz}), 6.44(\mathrm{~d}, 2 \mathrm{H}, J=8.5 \mathrm{~Hz}), 4.52(\mathrm{q}, 1 \mathrm{H}, J=6.5 \mathrm{~Hz}), 2.21(\mathrm{~s}, 3 \mathrm{H}), 1.54(\mathrm{~d}, 3 \mathrm{H}, J=$ $6.5 \mathrm{~Hz}) \mathrm{ppm}$.

${ }^{13} \mathrm{C}\left\{{ }^{1} \mathrm{H}\right\}$ NMR (125 MHz, $\left.\mathrm{CDCl}_{3}, 298 \mathrm{~K}\right): \delta 149.4,144.1,129.7,129.2\left(\mathrm{q}, \mathrm{J}_{\mathrm{CF}}=32.0 \mathrm{~Hz}\right), 127.2$, $126.3,125.6\left(\mathrm{q}, \mathrm{J}_{\mathrm{CF}}=3.8 \mathrm{~Hz}\right), 124.2\left(\mathrm{q}, \mathrm{J}_{\mathrm{CF}}=270.3 \mathrm{~Hz}\right), 113.7,53.8,24.8,20.3 \mathrm{ppm}$.

4-Methoxy-N-(1-phenylethyl)aniline ${ }^{1}$ (Table 2, Entry 5)<smiles>COc1ccc(NC(C)c2ccccc2)cc1</smiles>

The compound was prepared as described in the general procedure (Light Red solid, 87\% isolated yield, $108 \mathrm{mg}$ ).

${ }^{1} \mathrm{H}$ NMR $\left(400 \mathrm{MHz}, \mathrm{CDCl}_{3}, 298 \mathrm{~K}\right): \delta 7.24-7.42(\mathrm{~m}, 5 \mathrm{H}), 6.74(\mathrm{~d}, 2 \mathrm{H}, J=9.2 \mathrm{~Hz}), 6.51(\mathrm{~d}, 2 \mathrm{H}, J$ $=9.2 \mathrm{~Hz}), 4.45(\mathrm{q}, 1 \mathrm{H}, J=6.8 \mathrm{~Hz}), 3.73(\mathrm{~s}, 3 \mathrm{H}), 1.53(\mathrm{~d}, 3 \mathrm{H}, \mathrm{J}=6.9 \mathrm{~Hz}) \mathrm{ppm}$.

${ }^{13} \mathrm{C}\left\{{ }^{1} \mathrm{H}\right\}$ NMR (100 MHz, $\left.\mathrm{CDCl}_{3}, 298 \mathrm{~K}\right): \delta 151.9,145.5,141.6,128.6,126.8,125.8,114.7$, $114.5,55.7,54.2,25.0 \mathrm{ppm}$.

N-[1-(4-(Trifluoromethyl)phenyl)ethyl] aniline ${ }^{1}$ (Table 2, Entry 6)<smiles>CC(Nc1ccccc1)c1ccc(C(F)(F)F)cc1</smiles> 
The compound was prepared as described in the general procedure (Light yellow oil, $85 \%$ isolated yield, $112 \mathrm{mg}$ ).

${ }^{1} \mathrm{H}$ NMR (400 MHz, $\left.\mathrm{CDCl}_{3}, 298 \mathrm{~K}\right): \delta 7.58(\mathrm{~d}, 2 \mathrm{H}, J=8.0 \mathrm{~Hz}), 7.49(\mathrm{~d}, 2 \mathrm{H}, J=8.0 \mathrm{~Hz}$ ), 7.11 (t, $2 \mathrm{H}, J=7.3 \mathrm{~Hz}), 6.68(\mathrm{t}, 1 \mathrm{H}, J=7.3 \mathrm{~Hz}), 6.48(\mathrm{~d}, 2 \mathrm{H}, J=8.6 \mathrm{~Hz}), 4.54(\mathrm{q}, 1 \mathrm{H}, J=6.7 \mathrm{~Hz})$, 4.07 (brs, $1 \mathrm{H}), 1.53(\mathrm{~d}, 3 \mathrm{H}, J=6.7 \mathrm{~Hz}) \mathrm{ppm}$.

${ }^{13} \mathrm{C}\left\{{ }^{1} \mathrm{H}\right\}$ NMR $\left(100 \mathrm{MHz}, \mathrm{CDCl}_{3}, 298 \mathrm{~K}\right): \delta 149.5,146.8,129.2,129.2\left(\mathrm{q}, \mathrm{J}_{\mathrm{CF}}=33.4 \mathrm{~Hz}\right), 125.6$, $\left(\mathrm{q}, \mathrm{J}_{\mathrm{CF}}=3.8 \mathrm{~Hz}\right), 124.2\left(\mathrm{q}, \mathrm{J}_{\mathrm{CF}}=273.6 \mathrm{~Hz}\right), 117.7,113.3,53.3,25.1 \mathrm{ppm}$.

N-[1-(2-methylphenyl)ethyl]aniline ${ }^{1}$ (Table 2, Entry 7)

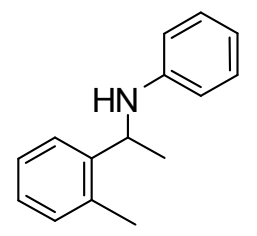

The compound was prepared as described in the general procedure (Yellow oil, 93\% isolated yield, $98 \mathrm{mg}$ ).

${ }^{1} \mathrm{H}$ NMR $\left(400 \mathrm{MHz}, \mathrm{CDCl}_{3}, 298 \mathrm{~K}\right): \delta$ 7.42-7.44 (m, $\left.1 \mathrm{H}\right), 7.13-7.16(\mathrm{~m}, 3 \mathrm{H}), 7.07-7.10(\mathrm{~m}, 2 \mathrm{H})$, 6.64 (t, 1H, $J=7.3 \mathrm{~Hz}$ ), 6.45 (d, 2H, $J=7.9 \mathrm{~Hz}$ ), 4.67 (q, 1H, $J=6.7 \mathrm{~Hz}$ ), 4.09 (brs, 1H), 2.44 $(\mathrm{s}, 3 \mathrm{H}), 1.48(\mathrm{~d}, 3 \mathrm{H}, \mathrm{J}=6.7 \mathrm{~Hz}) \mathrm{ppm}$.

${ }^{13} \mathrm{C}\left\{{ }^{1} \mathrm{H}\right\}$ NMR (125 MHz, $\left.\mathrm{CDCl}_{3}, 298 \mathrm{~K}\right): \delta 147.1,142.6,134.6,130.6,129.1,126.7,126.6$, $124.7,117.2,113.1,49.9,22.9,18.9 \mathrm{ppm}$.

$\mathbf{N}-\left[\mathbf{1}-\left(\mathbf{1 , 2 , 3 , 4 - t e t r a h y d r o n a p h t h a l e n y l ) a n i l i n e} \mathbf{e}^{1}\right.\right.$ (Table 2, Entry 8)

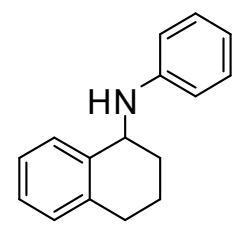

The compound was prepared as described in the general procedure (Yellow oil, 93\% isolated yield, $101 \mathrm{mg}$ ).

${ }^{1} \mathrm{H}$ NMR (500 MHz, $\mathrm{CDCl}_{3}, 298 \mathrm{~K}$ ): $\delta 7.45$ (d, $\left.1 \mathrm{H}, J=7.5 \mathrm{~Hz}\right), 7.16-7.24(\mathrm{~m}, 5 \mathrm{H}), 6.76(\mathrm{t}, 1 \mathrm{H}, J$ $=7.0 \mathrm{~Hz}), 6.71(\mathrm{~d}, 2 \mathrm{H}, J=8.0 \mathrm{~Hz}), 4.68(\mathrm{t}, 1 \mathrm{H}, J=4.5 \mathrm{~Hz}), 3.91(\mathrm{brs}, 1 \mathrm{H}), 2.78-2.92(\mathrm{~m}, 2 \mathrm{H})$, $1.82-2.03(\mathrm{~m}, 4 \mathrm{H}) \mathrm{ppm}$.

${ }^{13} \mathrm{C}\left\{{ }^{1} \mathrm{H}\right\}$ NMR $\left(125 \mathrm{MHz}, \mathrm{CDCl}_{3}, 298 \mathrm{~K}\right): \delta 147.4,138.1,137.6,129.3,129.2,129.0,127.1$, $126.0,117.0,112.8,51.0,29.3,28.7,19.4 \mathrm{ppm}$. 
General procedure for catalytic hydrosilylation of imines containing sugar moieties to secondary amines by $(a \mathrm{NHC}) \mathrm{Fe}(\mathrm{CO})_{4}$ :

A Schlenk tube was charged with the catalyst $1(0.001 \mathrm{mmol}, 0.71 \mathrm{mg}, 0.2 \mathrm{~mol} \%)$, aldimine or ketimine containing sugar reagents $(0.5 \mathrm{mmol}), \mathrm{PhSiH}_{3}(1.0 \mathrm{mmol}, 123.4 \mu \mathrm{L})$, and DMSO (1.0 $\mathrm{mL})$ or ethanol $(1.0 \mathrm{~mL})$ with magnetic stirring bar under air. The reaction mixture was stirred at room temperature for $12 \mathrm{~h}$. After the completion of the reaction, the reaction mixture was worked up with ether and water for aldimine containing sugar moieties and the combined organic fractions were dried under reduced pressure whereas for ketimine containing sugar derivative, after completion of the reaction, the reaction mixture was dried under reduced pressure. The conversion was determined by ${ }^{1} \mathrm{H}$ NMR. The amine was purified by using a silica gel chromatography column and a mixture of hexane/ethyl acetate (5-10\%) as the eluent.

\section{Characterization data for secondary amines bearing sugar moieties:}

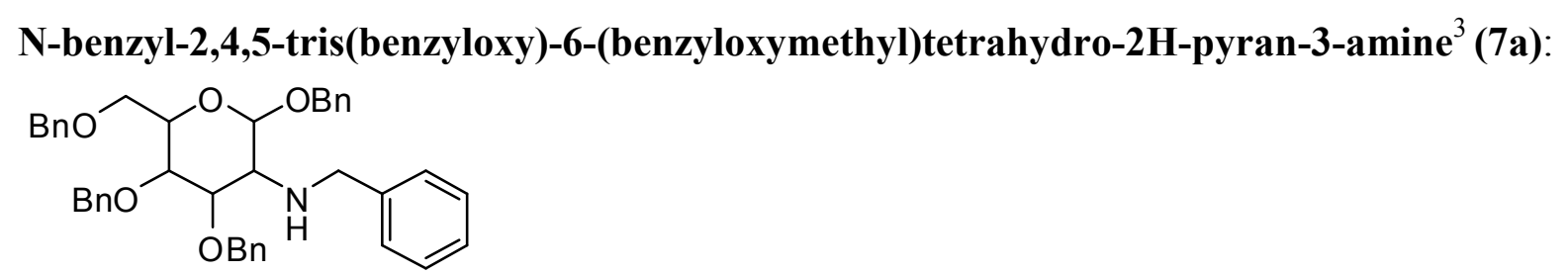

The compound was prepared as described in the general procedure (Colorlesssolid, $82 \%$ isolated yield, $258 \mathrm{mg}$ ).

${ }^{1} \mathrm{H}$ NMR (500 MHz, CDCl 3 -DMSO, $298 \mathrm{~K}$ ): $\delta$ 7.18-7.30 (m, 19H), 7.13-7.14 (m, 2H), 7.05-7.10 $(\mathrm{m}, 3 \mathrm{H}), 4.86-4.88(\mathrm{~m}, 1 \mathrm{H}), 4.75-4.77(\mathrm{~m}, 1 \mathrm{H}), 4.63-4.70(\mathrm{~m}, 2 \mathrm{H}), 4.51-4.59(\mathrm{~m}, 4 \mathrm{H}), 4.44-4.49$ (m, 2H), 3.62-3.75 (m, 4H), 3.46-3.56 (m, 2H), 3.36-3.41 (m, 1H) ppm.

${ }^{13} \mathrm{C}\left\{{ }^{1} \mathrm{H}\right\}$ NMR (125 MHz, $\mathrm{CDCl}_{3}$-DMSO, $\left.298 \mathrm{~K}\right): \delta 137.5,137.1,136.3,133.9,129.0,128.5$, $128.1,128.0,127.8,127.7,127.6,127.6,127.5,127.4,127.2,127.2,127.1,127.0,126.9,126.8$, $100.3,78.2,74.5,74.3,74.1,73.9,72.7,70.8,69.9,68.5,55.1 \mathrm{ppm}$.

\section{2,4,5-tris(benzyloxy)-6-(benzyloxymethyl)-N-(4-methylbenzyl)tetrahydro-2H-pyran-3-} amine (7b):<smiles>Cc1ccc(CNC2C(OCCCCC(=O)c3ccccc3)OC(COCc3ccccc3)C(OCc3ccccc3)C2OCc2ccccc2)cc1</smiles>

The compound was prepared as described in the general procedure (Colorless solid, $86 \%$ isolated yield, $276 \mathrm{mg}$ ).

${ }^{1} \mathrm{H}$ NMR $\left(500 \mathrm{MHz}, \mathrm{CDCl}_{3}, 298 \mathrm{~K}\right): \delta$ 7.28-7.37 (m, 16H), 7.22-7.24 (m, 4H), 7.16-7.18 (m, $3 \mathrm{H}), 7.11-7.12(\mathrm{~m}, 1 \mathrm{H}), 4.84-4.92(\mathrm{~m}, 3 \mathrm{H}), 4.69-4.70(\mathrm{~m}, 2 \mathrm{H}), 4.64-4.67(\mathrm{~m}, 2 \mathrm{H}), 4.62-4.63(\mathrm{~m}$, 
$1 \mathrm{H}), 4.59-4.60(\mathrm{~m}, 2 \mathrm{H}), 3.84-3.87(\mathrm{~m}, 1 \mathrm{H}), 3.79-3.82(\mathrm{~m}, 2 \mathrm{H}), 3.70-3.75(\mathrm{~m}, 2 \mathrm{H}), 3.64-3.68(\mathrm{~m}$, 1H), 3.39-3.44 (m, 1H), 2.44 (s, 3H) ppm.

${ }^{13} \mathrm{C}\left\{{ }^{1} \mathrm{H}\right\}$ NMR $\left(125 \mathrm{MHz}, \mathrm{CDCl}_{3}, 298 \mathrm{~K}\right): \delta 138.3,138.2,138.0,137.9,137.8,136.9,128.4$, $128.3,128.3,128.2,128.1,128.0,127.9,127.7,127.7,127.6,127.6,127.5,127.4,127.1,100.9$, $78.6,77.9,75.2,75.1,74.9,74.5,73.5,71.4,69.1,68.3,56.3,29.7 \mathrm{ppm}$.

ESI-MS: m/z calc. for $\mathrm{C}_{42} \mathrm{H}_{45} \mathrm{NO}_{5} \mathrm{H}[\mathrm{M}+\mathrm{H}]^{+}$644.3331, found 644.3330 .

\section{2,4,5-tris(benzyloxy)-6-(benzyloxymethyl)-N-(4-chlorobenzyl)tetrahydro-2H-pyran-3- amine (7c):}<smiles>OCC1OC(OCc2ccccc2)C(NCc2ccc(Cl)cc2)C(Cc2ccccc2)C1OCc1ccccc1</smiles>

The compound was prepared as described in the general procedure (Pale yellow coloredgel, $83 \%$ isolated yield, $275 \mathrm{mg}$ ).

${ }^{1} \mathrm{H}$ NMR $\left(500 \mathrm{MHz}, \mathrm{CDCl}_{3}, 298 \mathrm{~K}\right): \delta$ 7.31-7.40 (m, 15H), 7.21-7.25 (m, 4H), 7.17-7.19 (m, $3 \mathrm{H}), 7.09-7.13(\mathrm{~m}, 2 \mathrm{H}), 4.89$ (t, 2H, $J=10 \mathrm{~Hz}), 4.77$ (d, 1H, $J=8 \mathrm{~Hz}), 4.68-4.71(\mathrm{~m}, 3 \mathrm{H}), 4.61-$ $4.64(\mathrm{~m}, 3 \mathrm{H}), 4.52(\mathrm{~d}, 1 \mathrm{H}, J=10.5 \mathrm{~Hz}), 3.89-3.91(\mathrm{~m}, 2 \mathrm{H}), 3.81-3.83(\mathrm{~m}, 2 \mathrm{H}), 3.74-3.75(\mathrm{~m}, 1 \mathrm{H})$, 3.63-3.66 (m, 1H), $3.42(\mathrm{t}, 1 \mathrm{H}, J=8 \mathrm{~Hz}) \mathrm{ppm}$.

${ }^{13} \mathrm{C}\left\{{ }^{1} \mathrm{H}\right\}$ NMR $\left(125 \mathrm{MHz}, \mathrm{CDCl}_{3}, 298 \mathrm{~K}\right): \delta 137.5,137.1,136.3,133.9,129.0,128.5,128.1$, $128.0,127.8,127.7,127.6,127.6,127.5,127.4,127.2,127.2,127.1,127.0,126.9,126.8,100.3$, $78.2,74.5,74.3,74.1,73.9,72.7,70.8,69.9,68.5,55.1 \mathrm{ppm}$.

ESI-MS: m/z calc. for $\mathrm{C}_{41} \mathrm{H}_{42} \mathrm{ClNO}_{5} \mathrm{H}[\mathrm{M}+\mathrm{H}]^{+}$664.2785, found 664.2801.

\section{2,4,5-tris(benzyloxy)-6-(benzyloxymethyl)-N-((5-methylthiophen-2-yl)methyl)tetrahydro-} 2H-pyran-3-amine (7d):<smiles>Cc1ccc(CNC2C(OCc3ccccc3)OC(COCc3ccccc3)C(OCc3ccccc3)C2COc2ccccc2)s1</smiles>

The compound was prepared as described in the general procedure (Pale yellow colored gel, $92 \%$ isolated yield, $298 \mathrm{mg}$ ).

${ }^{1} \mathrm{H}$ NMR $\left(500 \mathrm{MHz}, \mathrm{CDCl}_{3}, 298 \mathrm{~K}\right): \delta$ 7.28-7.37 (m, 16H), 7.22-7.24 (m, 4H), 7.16-7.18 (m, $3 \mathrm{H}), 7.11-7.12(\mathrm{~m}, 1 \mathrm{H}), 4.84-4.92(\mathrm{~m}, 3 \mathrm{H}), 4.69-4.70(\mathrm{~m}, 2 \mathrm{H}), 4.64-4.67(\mathrm{~m}, 2 \mathrm{H}), 4.62-4.63(\mathrm{~m}$, 
$1 \mathrm{H}), 4.59-4.60(\mathrm{~m}, 2 \mathrm{H}), 3.84-3.87(\mathrm{~m}, 1 \mathrm{H}), 3.79-3.82(\mathrm{~m}, 2 \mathrm{H}), 3.70-3.75(\mathrm{~m}, 2 \mathrm{H}), 3.64-3.68(\mathrm{~m}$, $1 \mathrm{H}), 3.39-3.44(\mathrm{~m}, 1 \mathrm{H}), 2.44(\mathrm{~s}, 3 \mathrm{H}) \mathrm{ppm}$.

${ }^{13} \mathrm{C}\left\{{ }^{1} \mathrm{H}\right\}$ NMR (125 MHz, $\left.\mathrm{CDCl}_{3}, 298 \mathrm{~K}\right): \delta 138.3,138.2,138.0,137.9,137.8,136.9,128.4$, $128.3,128.3,128.2,128.1,128.0,127.9,127.7,127.7,127.6,127.6,127.5,127.4,127.1,100.9$, 78.6, 77.9, 75.2, 75.1, 74.9, 74.5, 73.5, 71.4, 69.1, 68.3, 56.3, $29.7 \mathrm{ppm}$.

ESI-MS: $\mathrm{m} / \mathrm{z}$ calc. for $\mathrm{C}_{40} \mathrm{H}_{43} \mathrm{NO}_{5} \mathrm{SH}[\mathrm{M}+\mathrm{H}]^{+}$650.2895, found 650.2900 .

\section{X-ray crystallographic details.}

Single crystals of the compounds $\mathbf{1}$ was mounted on a glass pip. Intensity data were collected on a SuperNova, Dual, Mo at zero, Eos diffractometer. The crystals were kept at 100K during data collection. Atomic coordinates, isotropic and anisotropic displacement parameters of all the nonhydrogen atoms of two compounds were refined using Olex $2,{ }^{4}$ and the structure was solved with the Superflip ${ }^{5}$ structure solution program using Charge Flipping and refined with the ShelXL ${ }^{6}$ refinement package using Least Squares minimization. Structure graphics shown in the figures were created using the Olex2 and X-Seed software package version 2.0. ${ }^{7}$

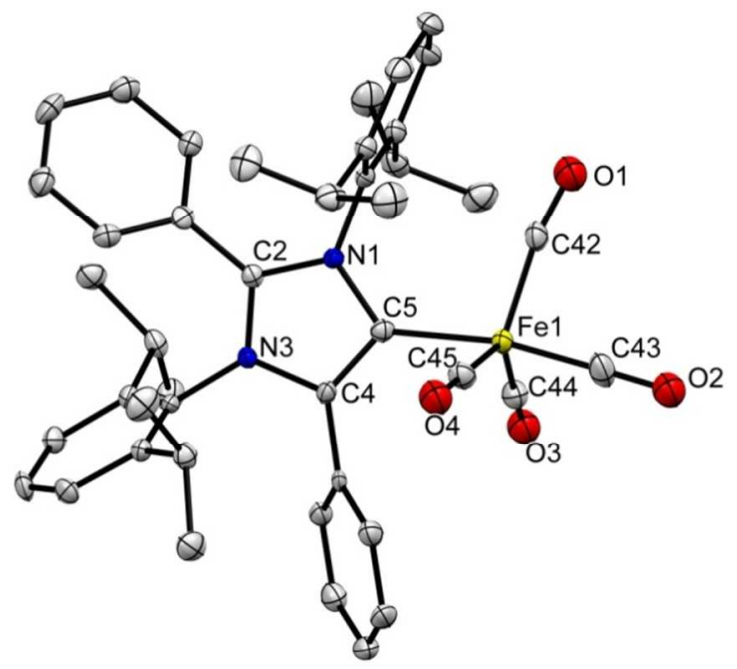

Figure S1. View of the molecular structure of 1 . Ellipsoids are set at 50\% probability; hydrogen atoms have been omitted for clarity. 
Table S3.Selected bond distances $(\AA \AA)$ and angles $\left(^{\circ}\right)$ observed in 1

\begin{tabular}{|l|l|l|l|}
\hline Bond & Distance & \multicolumn{1}{|c|}{ Bond } & \multicolumn{1}{c|}{ Angles } \\
\hline $\mathrm{Fe}(1)-\mathrm{C}(5)$ & $2.027(3)$ & $\mathrm{C}(5)-\mathrm{Fe}(1)-\mathrm{C}(42)$ & $101.86(15)$ \\
\hline $\mathrm{Fe}(1)-\mathrm{C}(42)$ & $1.796(4)$ & $\mathrm{C}(5)-\mathrm{Fe}(1)-\mathrm{C}(43)$ & $169.71(16)$ \\
\hline $\mathrm{Fe}(1)-\mathrm{C}(43)$ & $1.745(4)$ & $\mathrm{C}(5)-\mathrm{Fe}(1)-\mathrm{C}(44)$ & $84.82(15)$ \\
\hline $\mathrm{Fe}(1)-\mathrm{C}(44)$ & $1.757(4)$ & $\mathrm{C}(5)-\mathrm{Fe}(1)-\mathrm{C}(45)$ & $85.27(17)$ \\
\hline $\mathrm{Fe}(1)-\mathrm{C}(45)$ & $1.770(4)$ & $\mathrm{C}(42)-\mathrm{Fe}(1)-\mathrm{C}(43)$ & $88.43(17)$ \\
\hline $\mathrm{O}(1)-\mathrm{C}(42)$ & $1.164(5)$ & $\mathrm{C}(42)-\mathrm{Fe}(1)-\mathrm{C}(44)$ & $114.38(17)$ \\
\hline $\mathrm{O}(2)-\mathrm{C}(43)$ & $1.174(5)$ & $\mathrm{C}(42)-\mathrm{Fe}(1)-\mathrm{C}(45)$ & $115.28(17)$ \\
\hline $\mathrm{O}(3)-\mathrm{C}(44)$ & $1.166(5)$ & $\mathrm{C}(43)-\mathrm{Fe}(1)-\mathrm{C}(44)$ & $90.71(17)$ \\
\hline $\mathrm{O}(4)-\mathrm{C}(45)$ & $1.164(5)$ & $\mathrm{C}(43)-\mathrm{Fe}(1)-\mathrm{C} 45)$ & $90.6(2)$ \\
\hline $\mathrm{N}(1)-\mathrm{C}(5)$ & $1.416(4)$ & $\mathrm{C}(44)-\mathrm{Fe}(1)-\mathrm{C}(45)$ & $130.35(17)$ \\
\hline & & $\mathrm{Fe}(1)-\mathrm{C}(5)-\mathrm{N}(1)$ & $127.8(2)$ \\
\hline & & $\mathrm{Fe}(1)-\mathrm{C}(5)-\mathrm{C}(4)$ & $128.5(2)$ \\
\hline
\end{tabular}

Table S4. Crystallographic data andsummary of data collection and structure refinement for complex 1 .

\begin{tabular}{|l|l|}
\hline \multicolumn{1}{|c|}{ CCDC No } & 1451782 \\
\hline Formula & $\mathrm{C}_{43} \mathrm{H}_{44} \mathrm{Fe} \mathrm{N}_{2} \mathrm{O}_{4}$ \\
\hline Crystal System & Triclinic \\
\hline Space group & $P-1$ \\
\hline$a[\AA]$ & $10.1393(8)$ \\
\hline$b[\AA]$ & $10.7878(10)$ \\
\hline$c[\AA]$ & $18.1314(15)$ \\
\hline$\alpha\left[^{0}\right]$ & $77.341(8)$ \\
\hline$\beta\left[^{0}\right]$ & $80.516(7)$ \\
\hline$\gamma\left[^{0}\right]$ & $79.342(7)$ \\
\hline$V\left[^{0} \AA^{3}\right]$ & $1885.6(3)$ \\
\hline$Z$ & 2 \\
\hline
\end{tabular}




\begin{tabular}{|l|l|}
\hline$\lambda[\AA]$ & 0.71073 \\
\hline$\rho_{\text {calcd }}\left[\mathrm{gcm}^{-3}\right]$ & 1.248 \\
\hline$F[000]$ & 748.0 \\
\hline$\mu\left[\mathrm{mm}^{-1}\right]$ & 0.443 \\
\hline$\theta\left[^{0}\right]$ & $1.96-25.03$ \\
\hline index ranges & $-12 \leq \mathrm{h} \leq 12$ \\
& $-12 \leq \mathrm{k} \leq 12$ \\
\hline$T[\mathrm{~K}]$ & $-20 \leq 1 \leq 21$ \\
\hline$R 1$ & 100 \\
\hline$w R 2$ & 0.0529 \\
\hline$R_{\text {merge }}$ & 0.1225 \\
\hline Parameters & 0.0823 \\
\hline GOF & 459 \\
\hline reflns total & 1.045 \\
\hline unique reflns & 11853 \\
\hline obsdreflns & 6654 \\
\hline & 5162 \\
\hline & \\
\hline & \\
\hline & \\
\hline
\end{tabular}




\section{${ }^{1}$ H NMR and ${ }^{13} \mathrm{C}$ NMR spectra.}

Figure S2. ${ }^{1} \mathrm{H}$ NMR and ${ }^{13} \mathrm{C}$ NMR spectra of complex $\mathbf{1}$ in $\mathrm{C}_{6} \mathrm{D}_{6}$

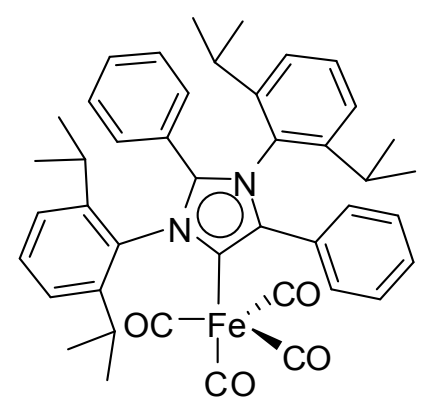

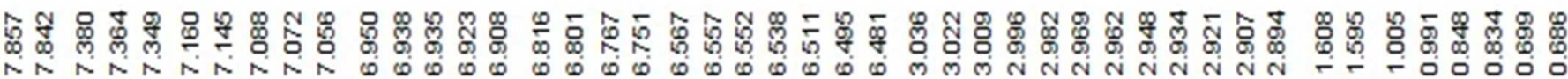
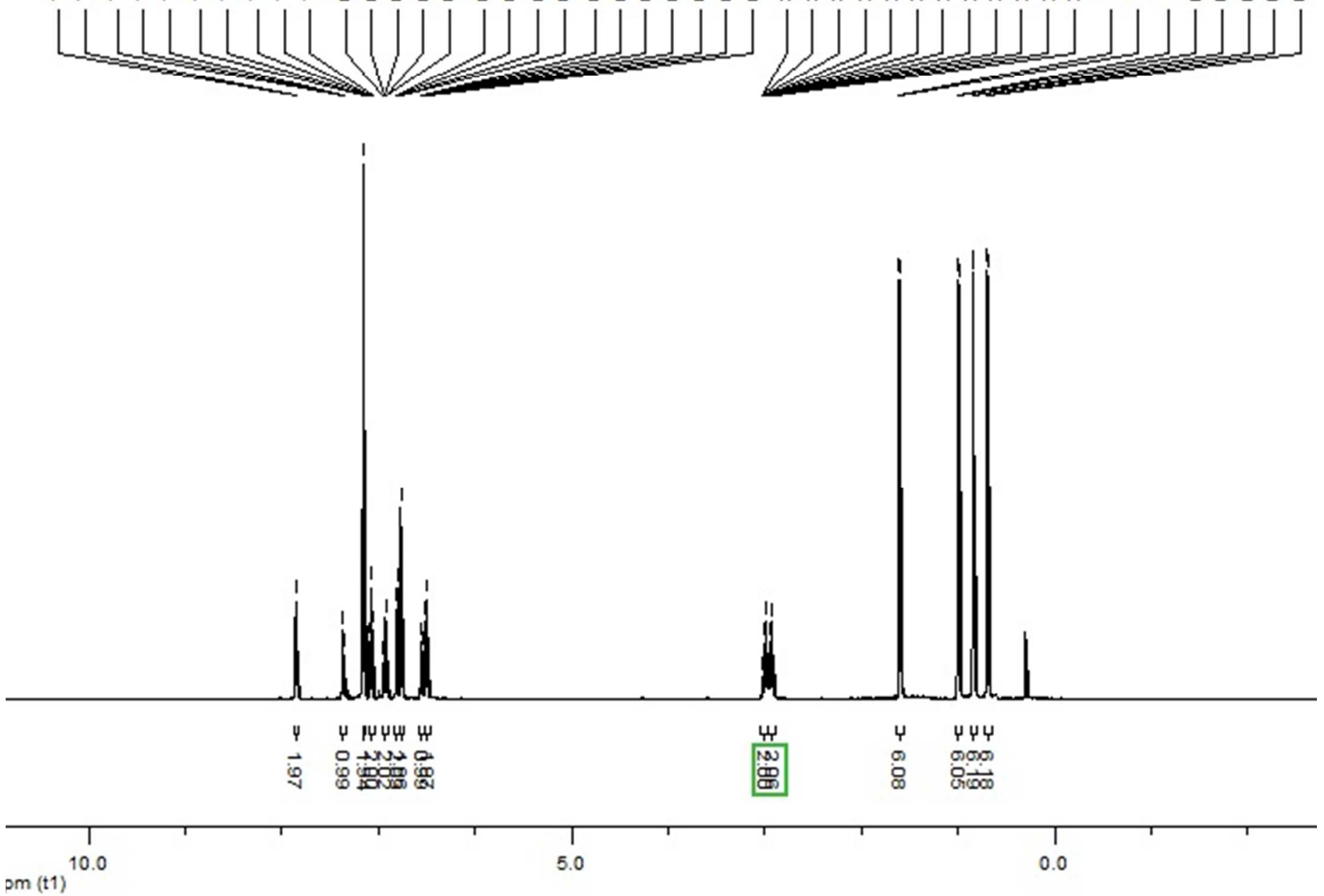

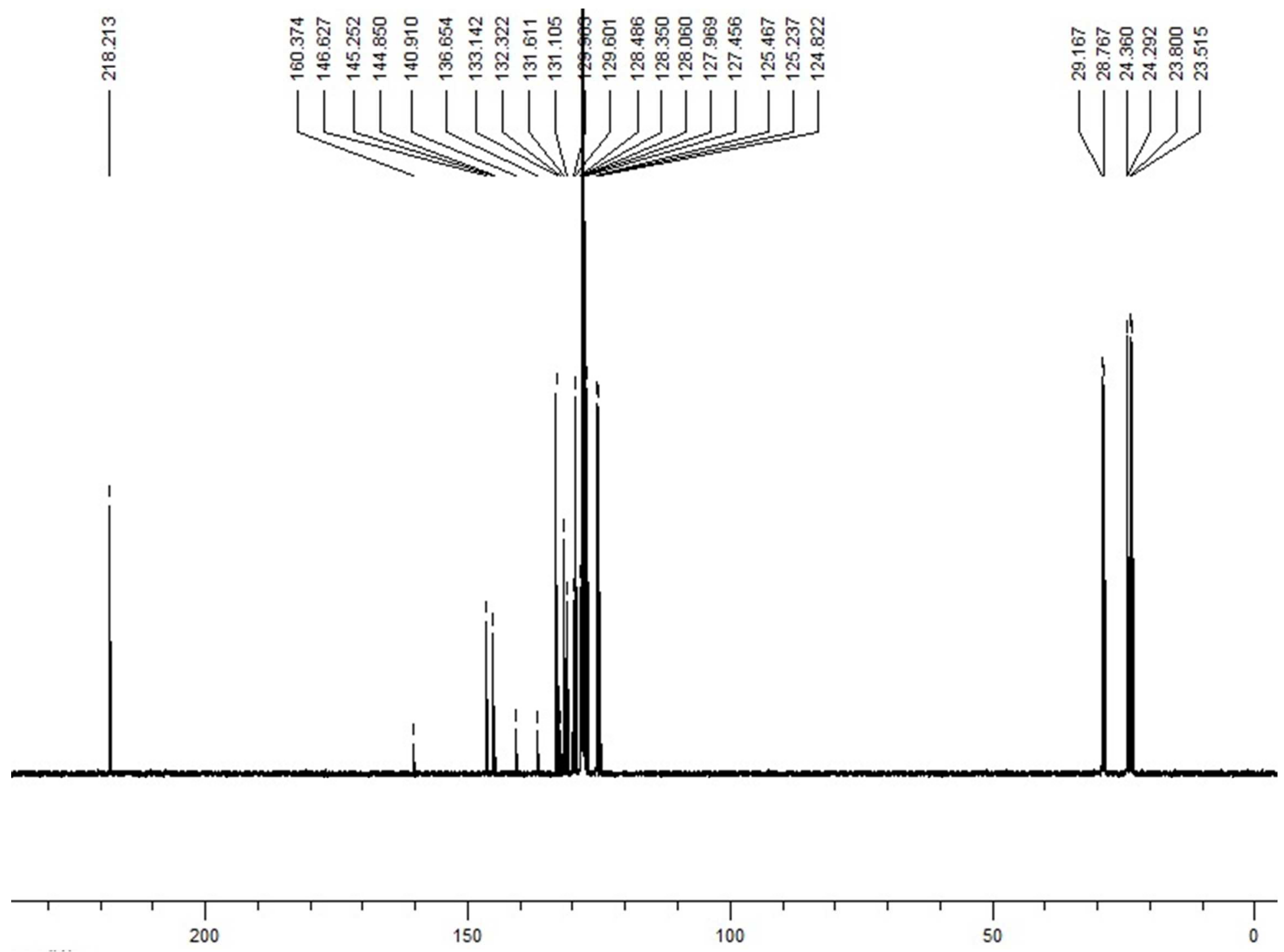
Figure S3. ${ }^{1} \mathrm{H}$ NMR, ${ }^{13} \mathrm{C}$ NMR and ${ }^{29} \mathrm{Si}$ NMR spectra of complex 9 in $\mathrm{C}_{6} \mathrm{D}_{6}$
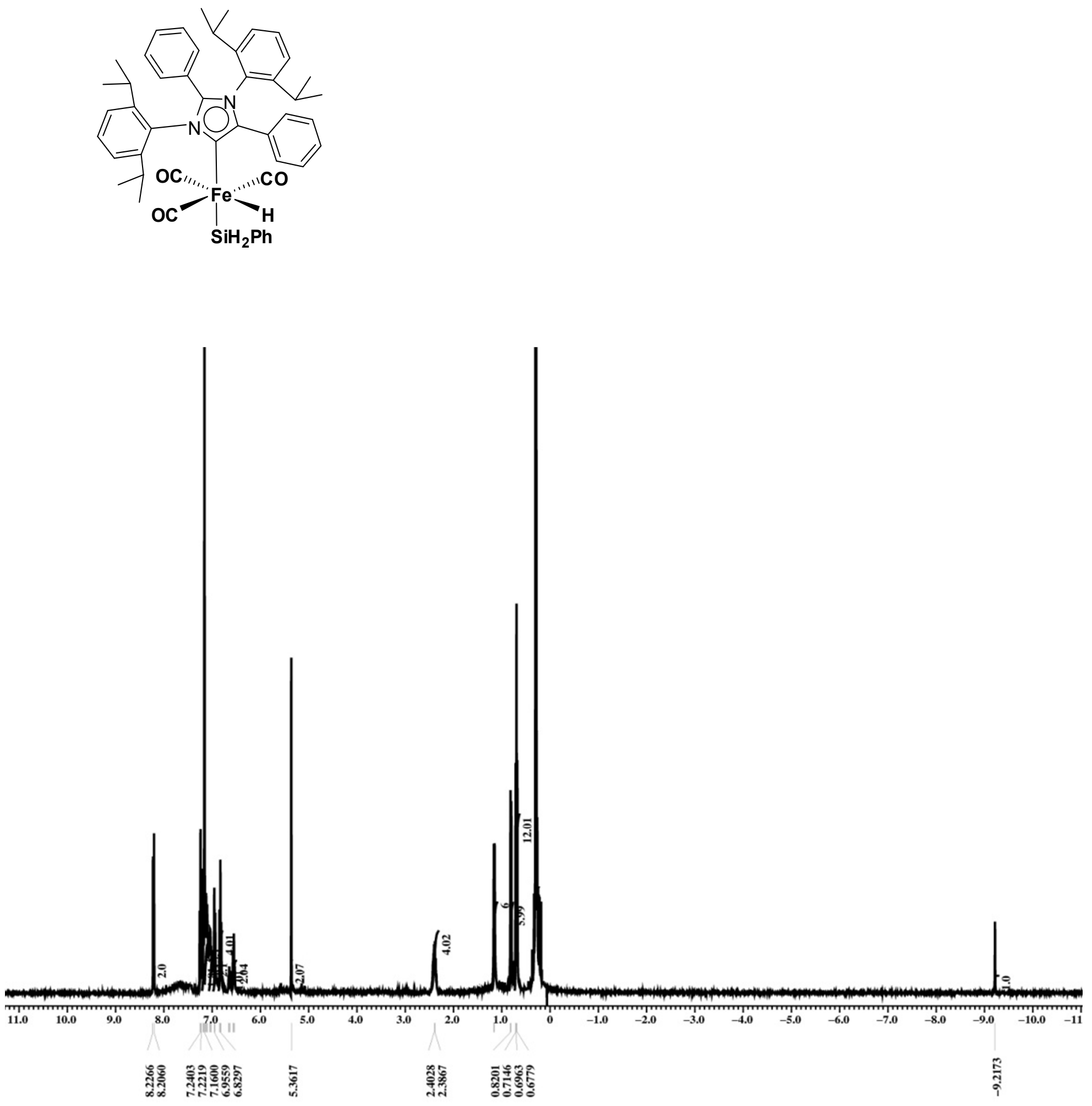

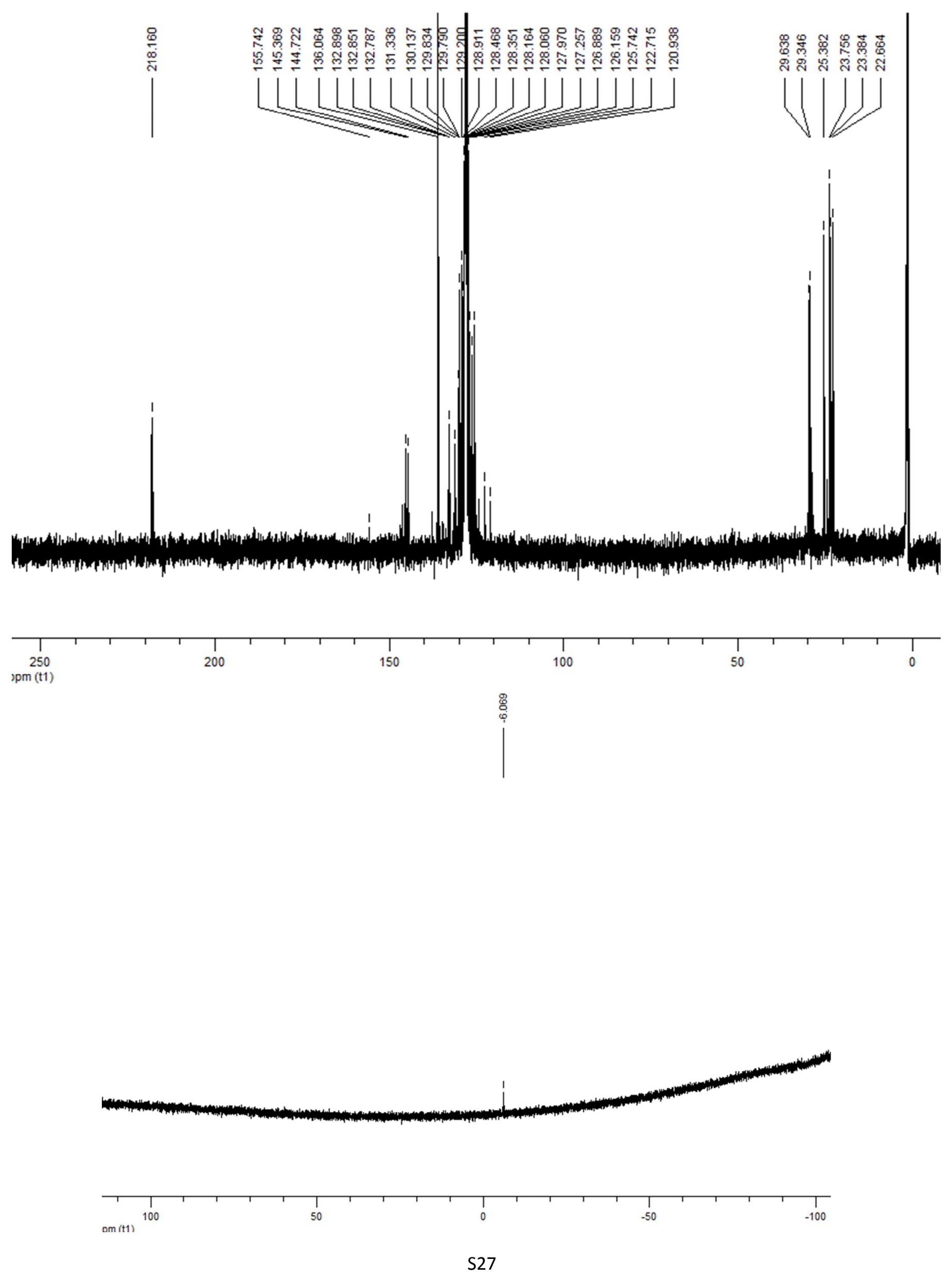
Figure S4. ${ }^{1} \mathrm{H}$ NMR and ${ }^{13} \mathrm{C}$ NMR spectra of complex 11 in DMSO-d $\mathrm{d}_{6}$

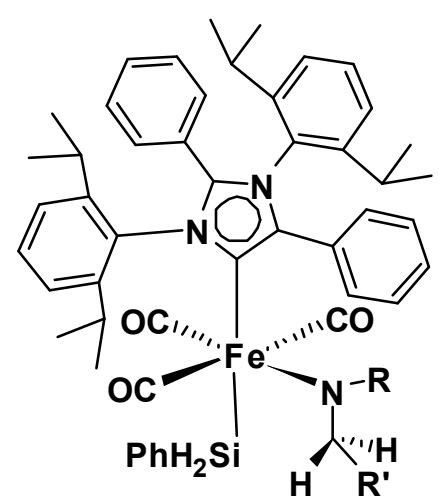

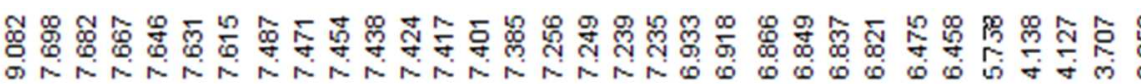
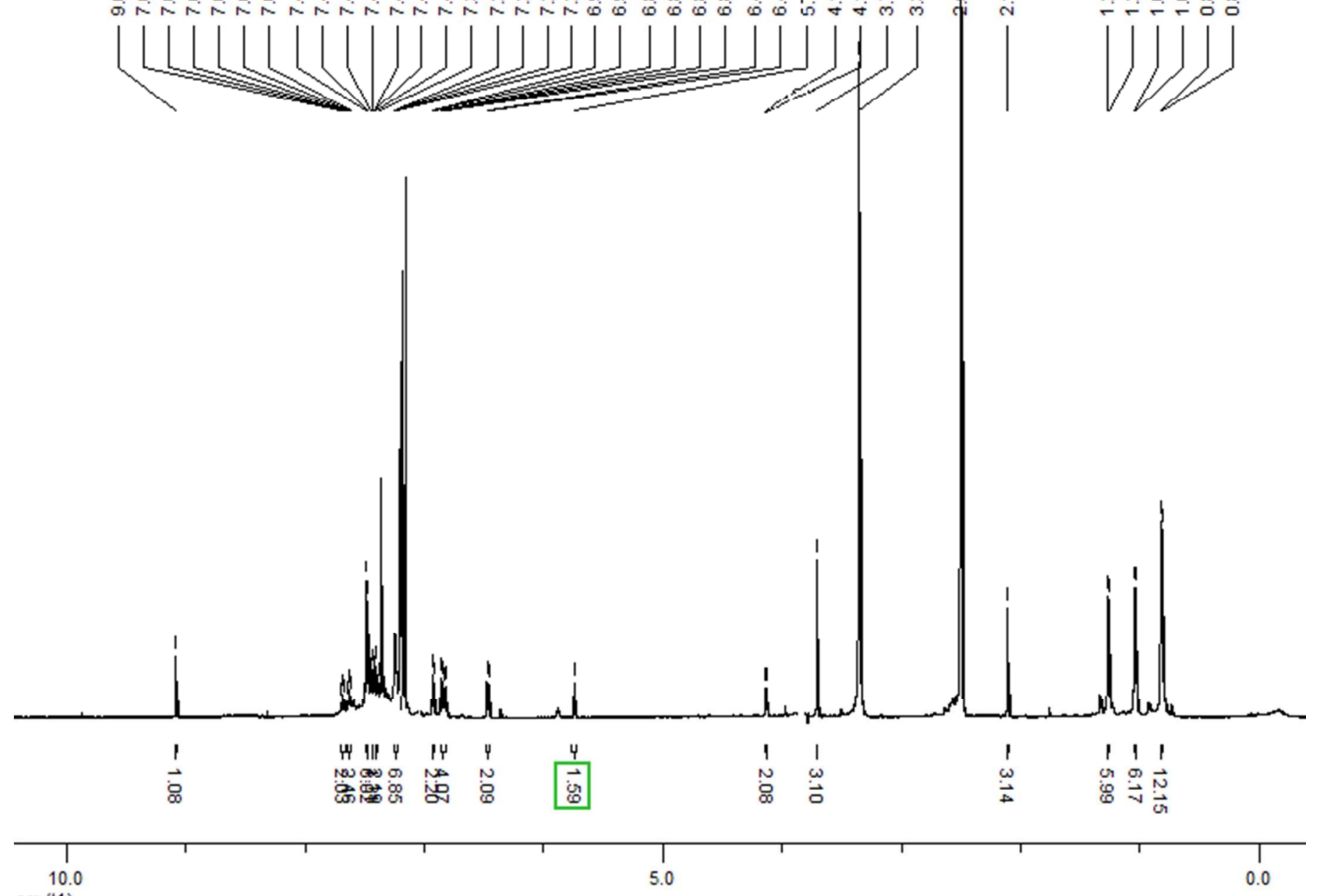


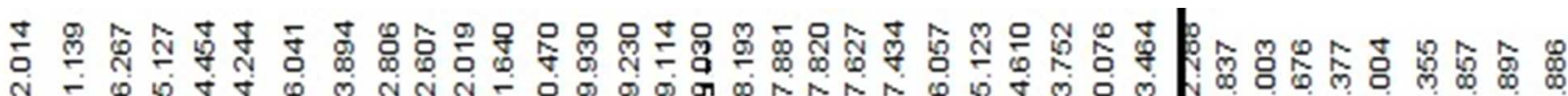

Nูำ
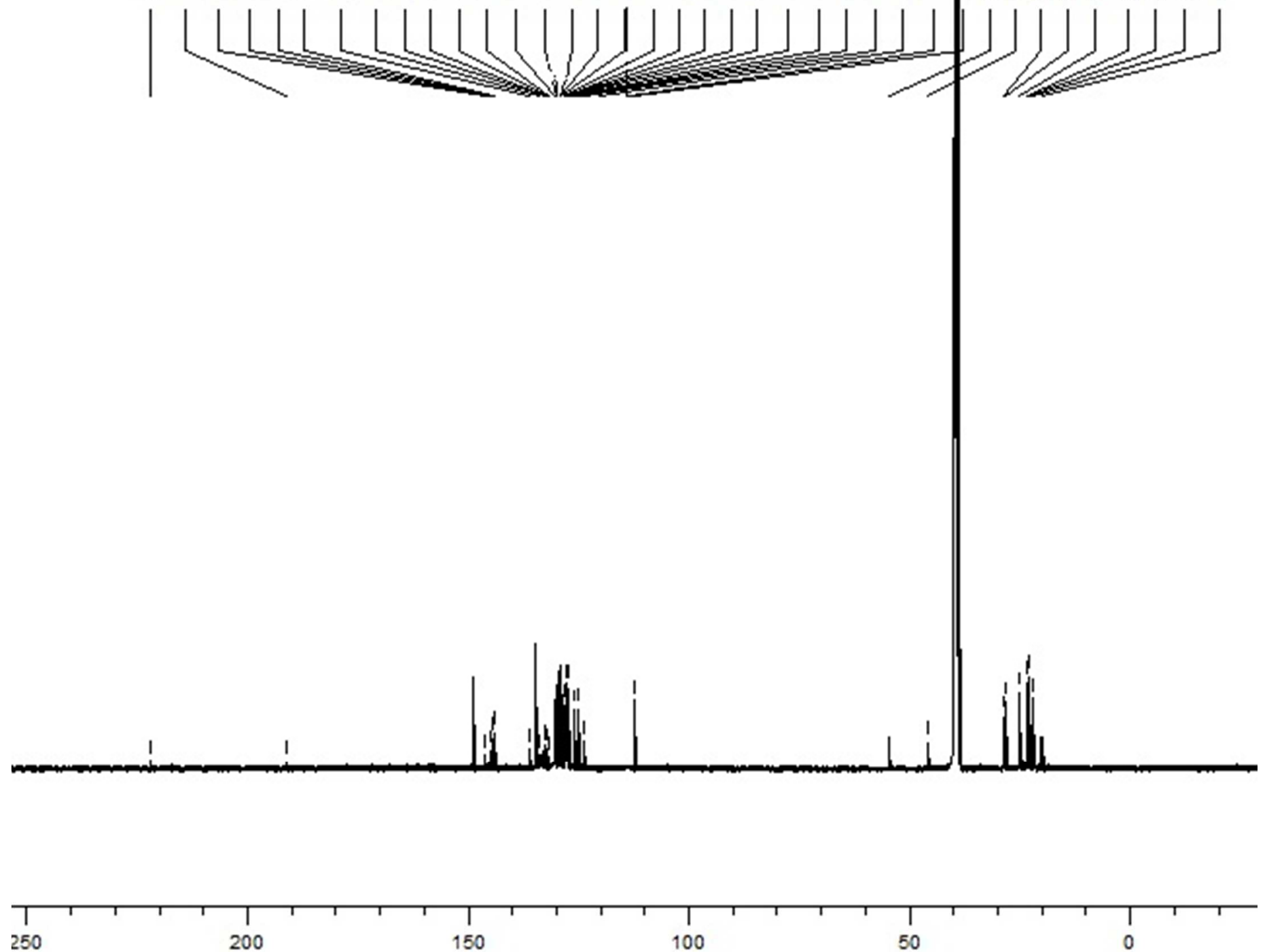
Figure S5. ${ }^{1} \mathrm{H}$ NMR and ${ }^{13} \mathrm{C}$ NMR spectra of N-benzylaniline in $\mathrm{CDCl}_{3}$ (Table 1, Entry 1 )<smiles>c1ccc(CNc2ccccc2)cc1</smiles>

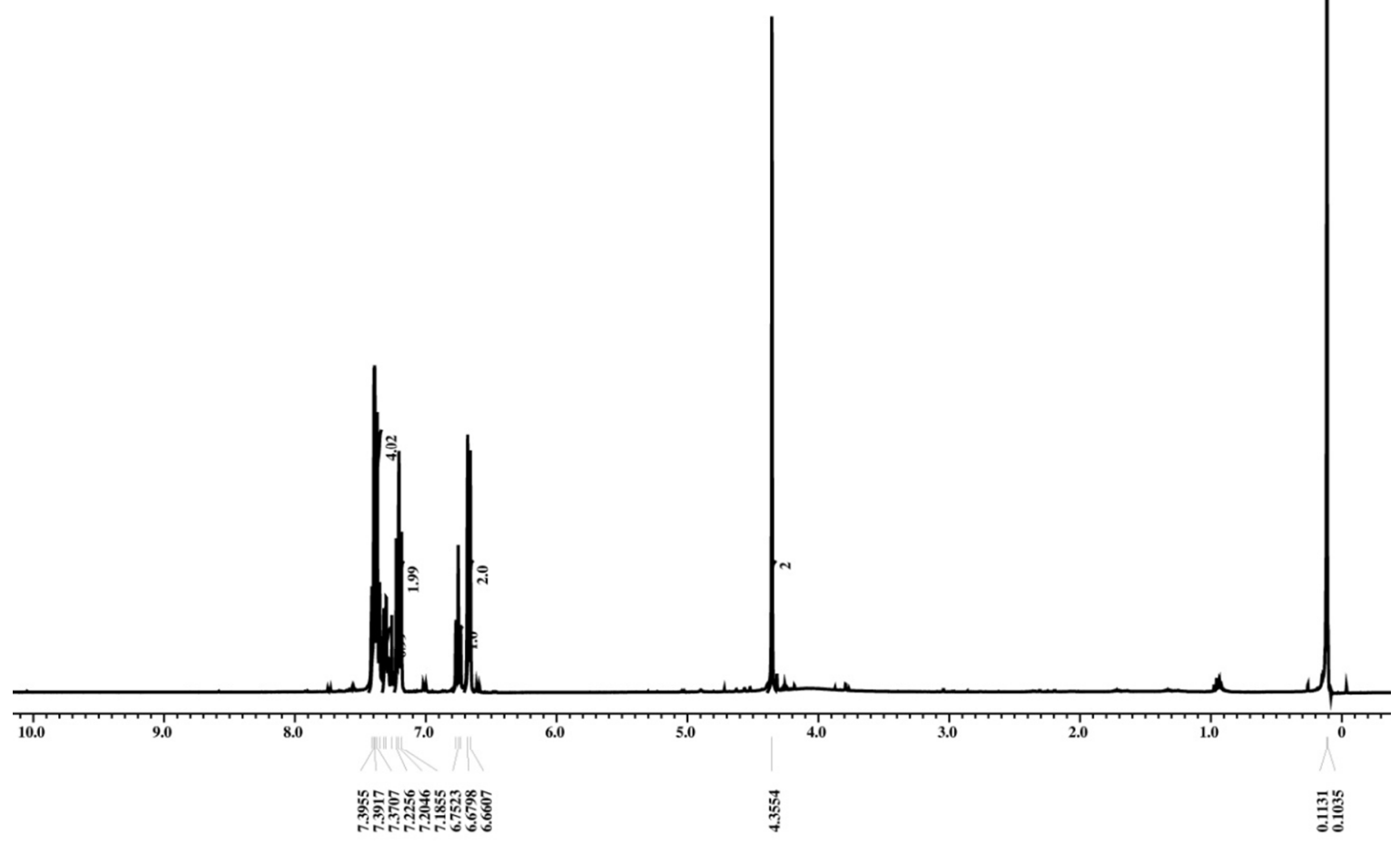




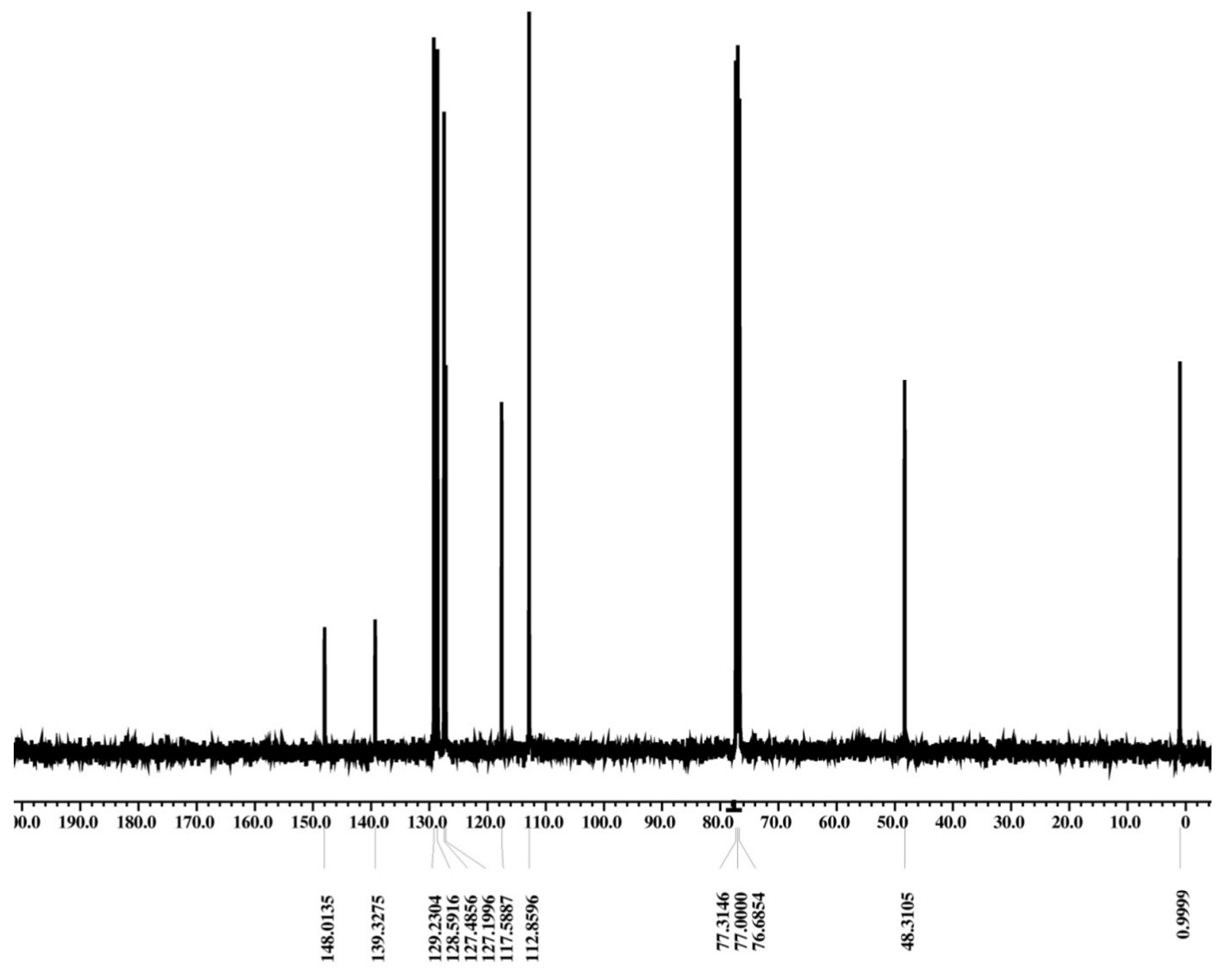


Figure S6. ${ }^{1} \mathrm{H}$ NMR and ${ }^{13} \mathrm{C}$ NMR spectra of N-benzyl-4-methylaniline in $\mathrm{CDCl}_{3}$ (Table 1, Entry 2)<smiles>[TeH2]</smiles>

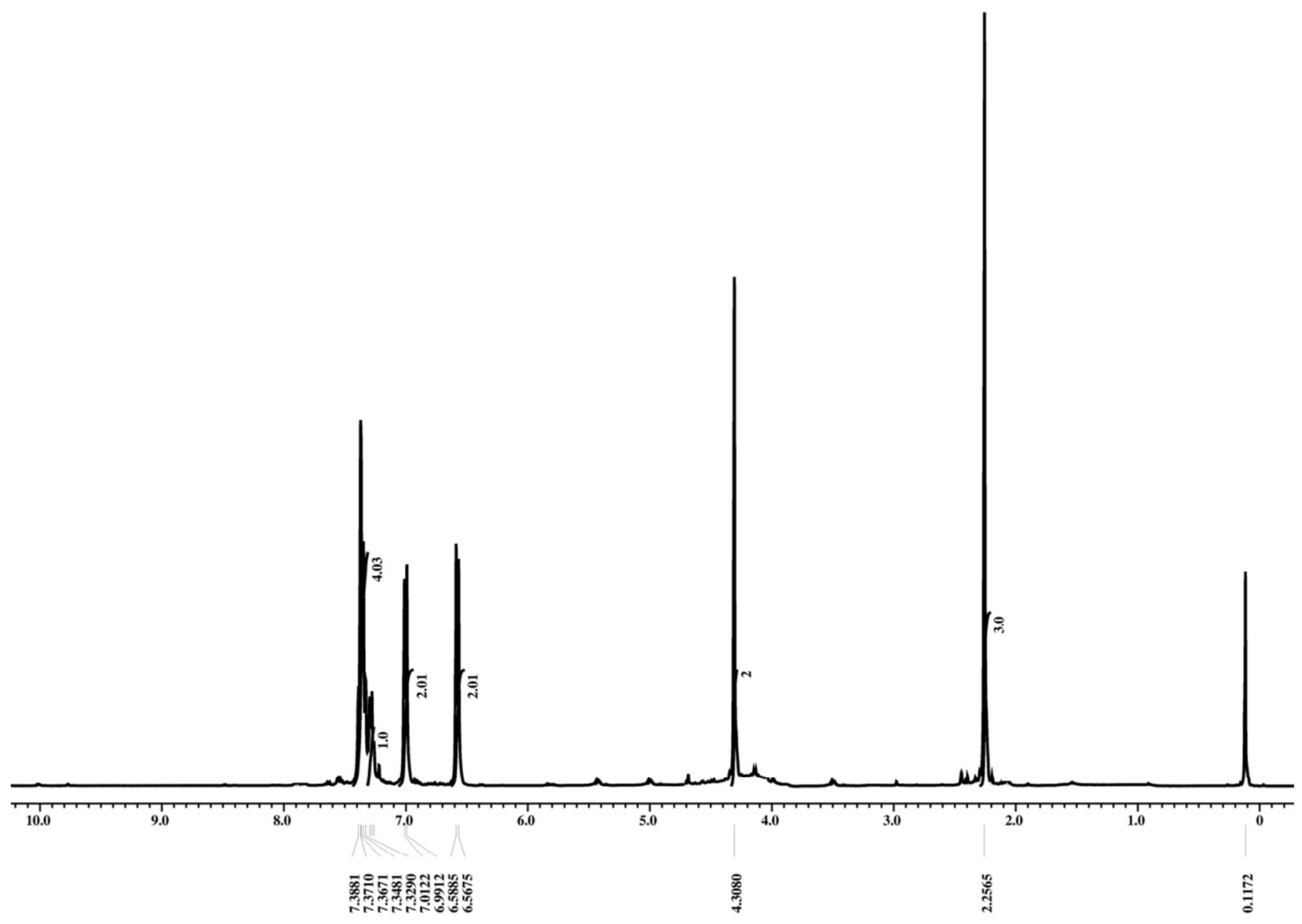




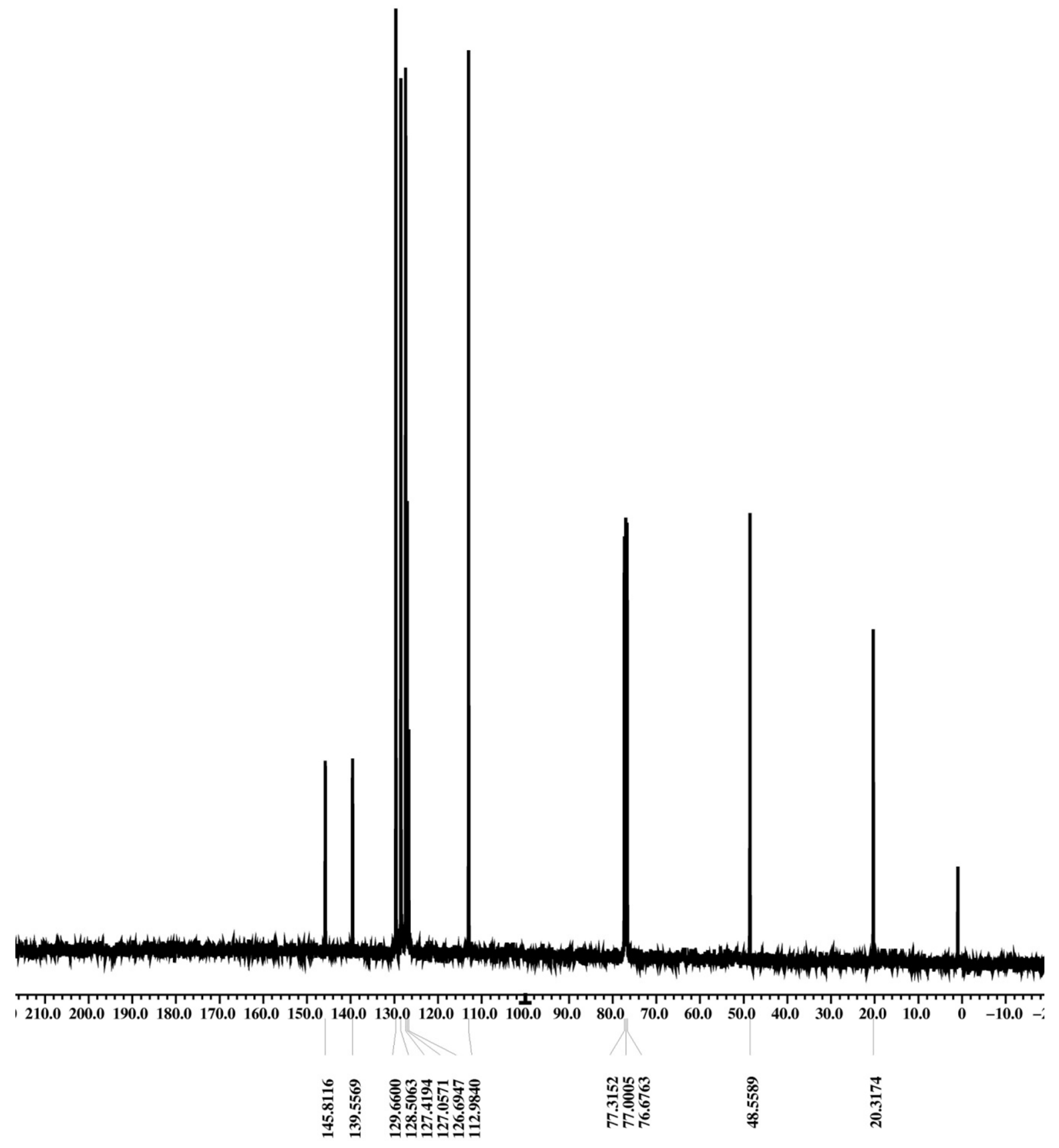


Figure S7. ${ }^{1} \mathrm{H}$ NMR and ${ }^{13} \mathrm{C}$ NMR spectra of N-Benzyl-2-methylanilinein $\mathrm{CDCl}_{3}$ (Table 1, Entry 3)<smiles>Cc1ccccc1NCc1ccccc1</smiles>

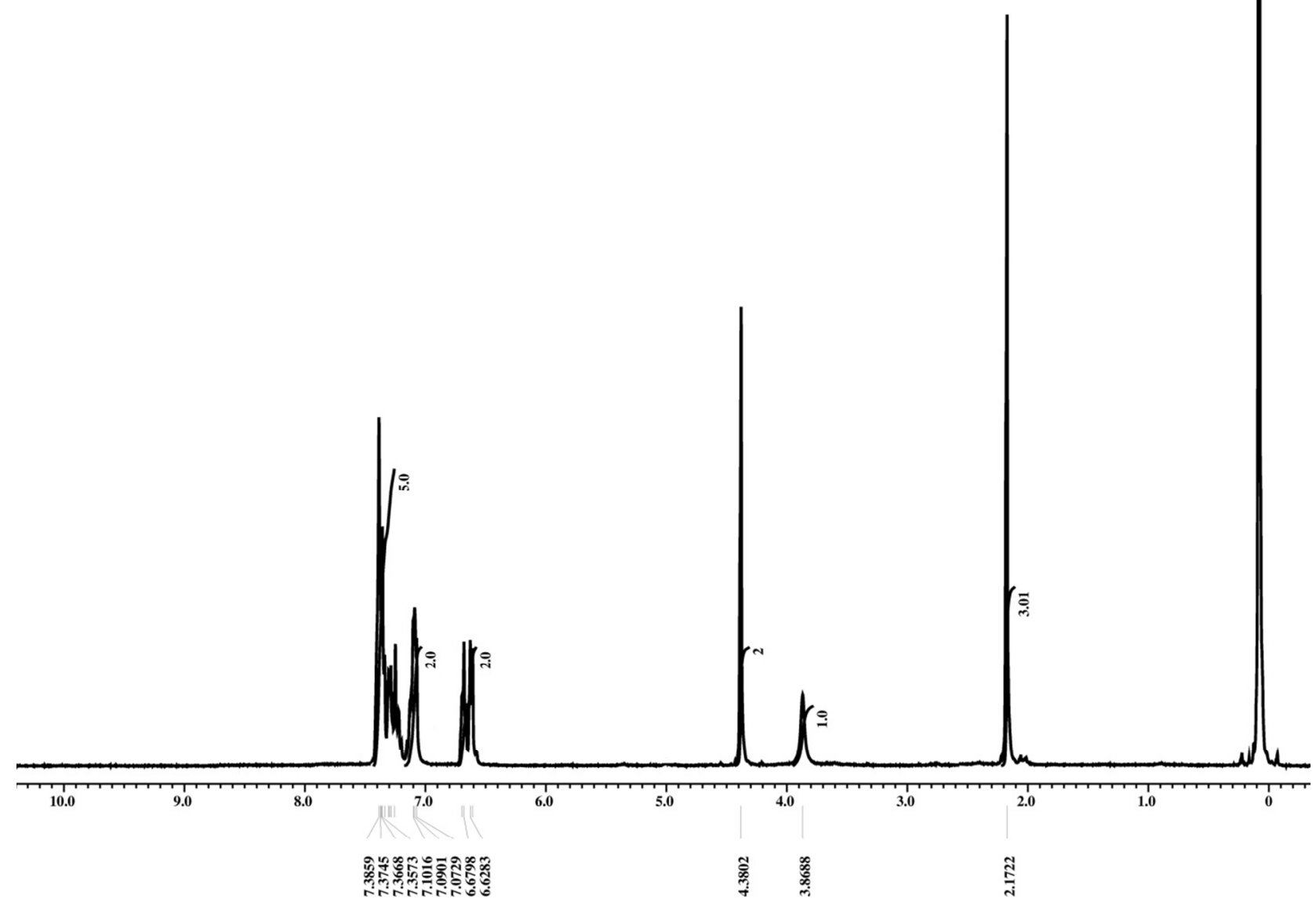




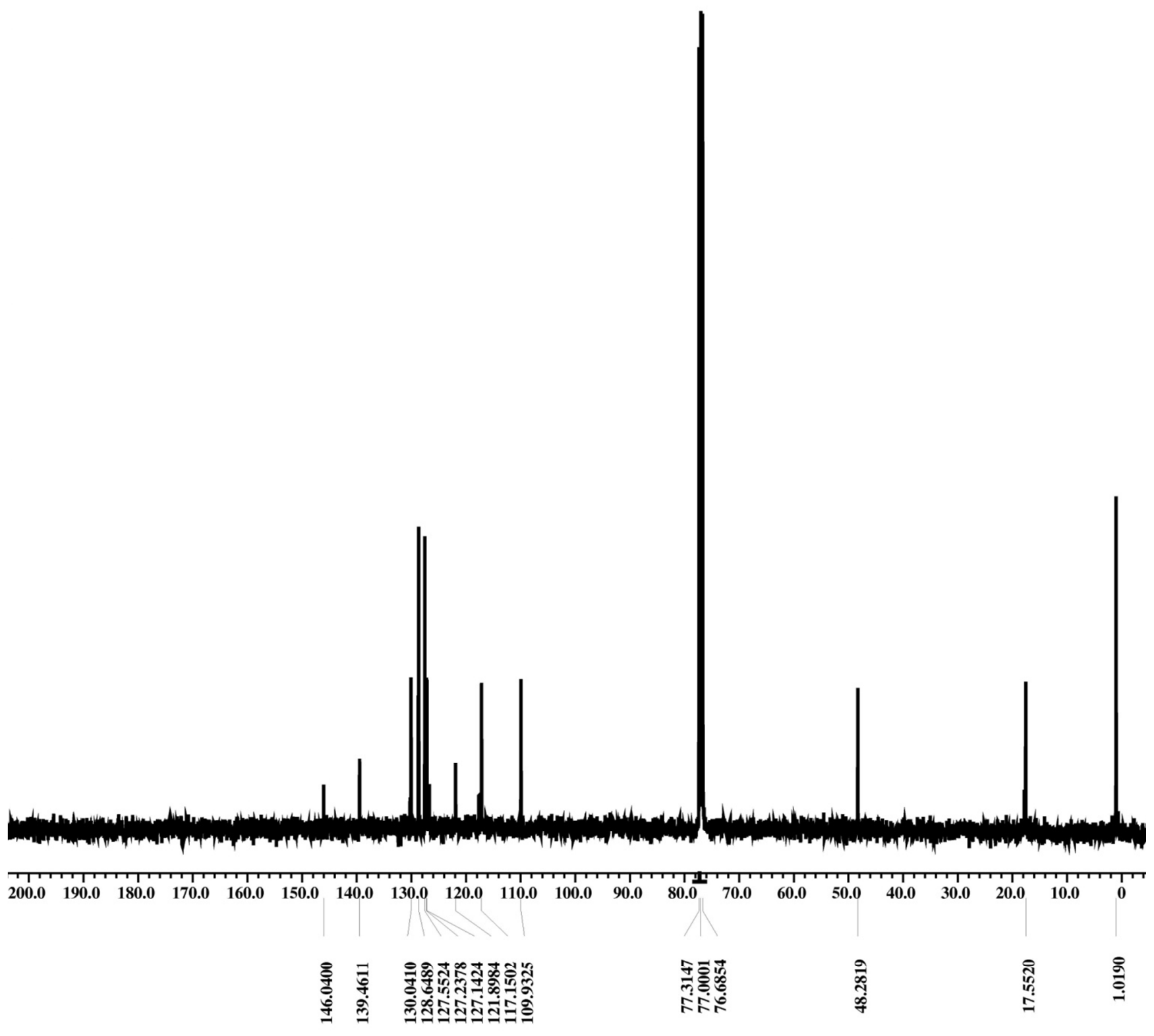


Figure S8. ${ }^{1} \mathrm{H}$ NMR and ${ }^{13} \mathrm{C}$ NMR spectra of N-(4-Methylbenzyl)-4-methylaniline in $\mathrm{CDCl}_{3}$ (Table 1, Entry 5)<smiles>Cc1ccc(CNc2ccc(C)cc2)cc1</smiles>

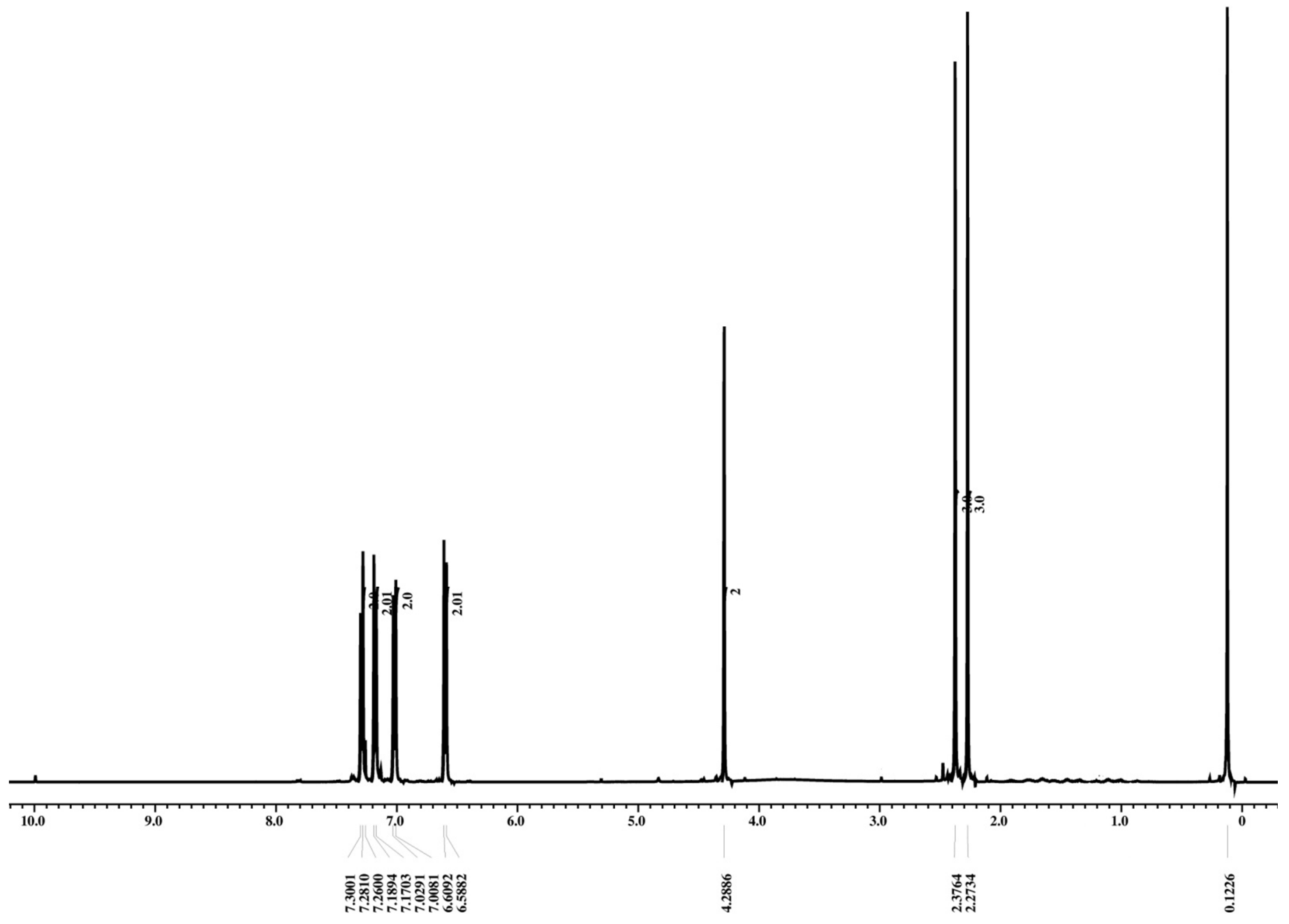




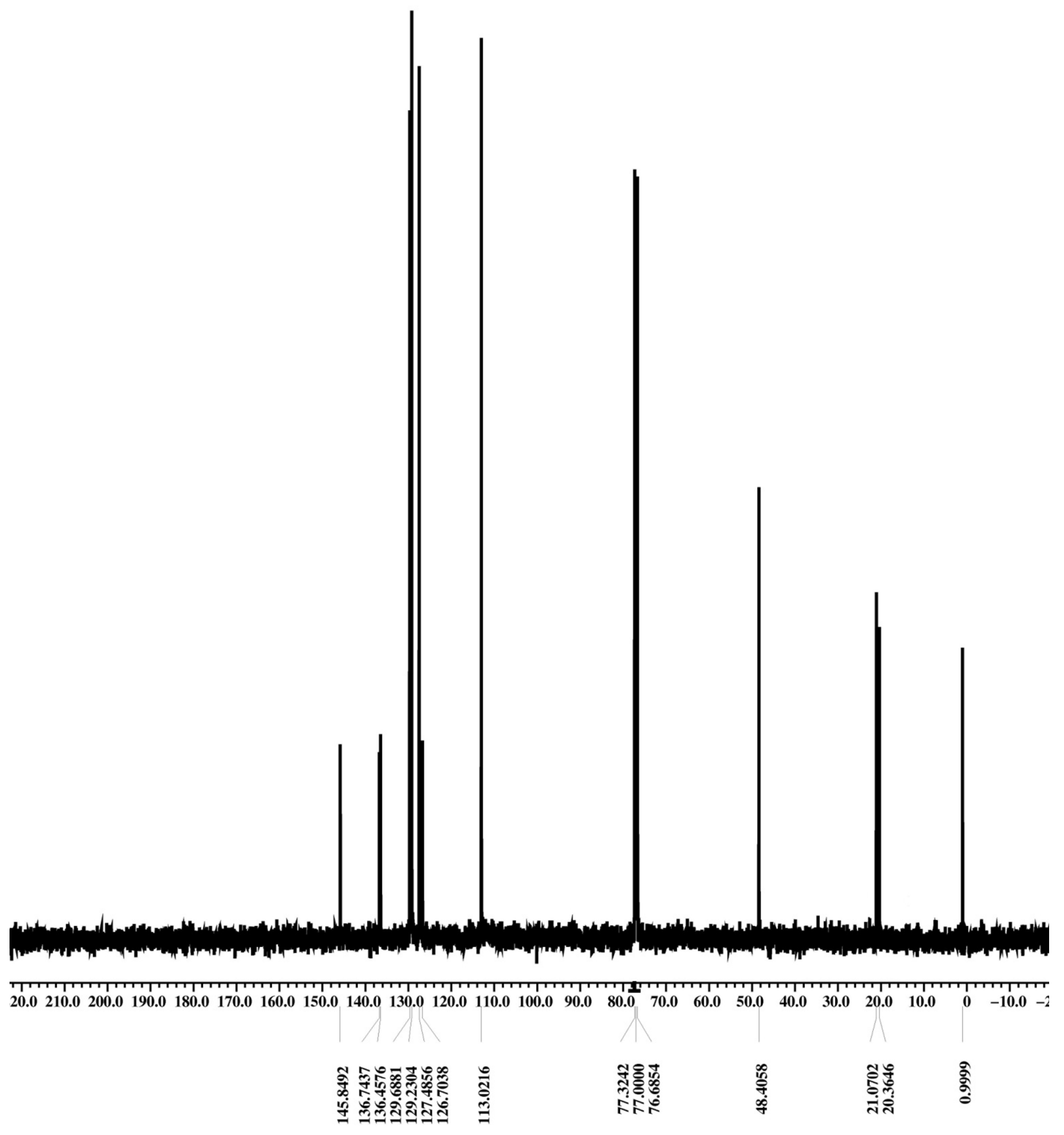


Figure S9. ${ }^{1} \mathrm{H}$ NMR and ${ }^{13} \mathrm{C}$ NMR spectra of N-(4-Methoxybenzyl)-4-methylaniline in $\mathrm{CDCl}_{3}$ (Table 1, Entry 6)<smiles>COc1ccc(CNc2ccc(C)cc2)cc1</smiles>

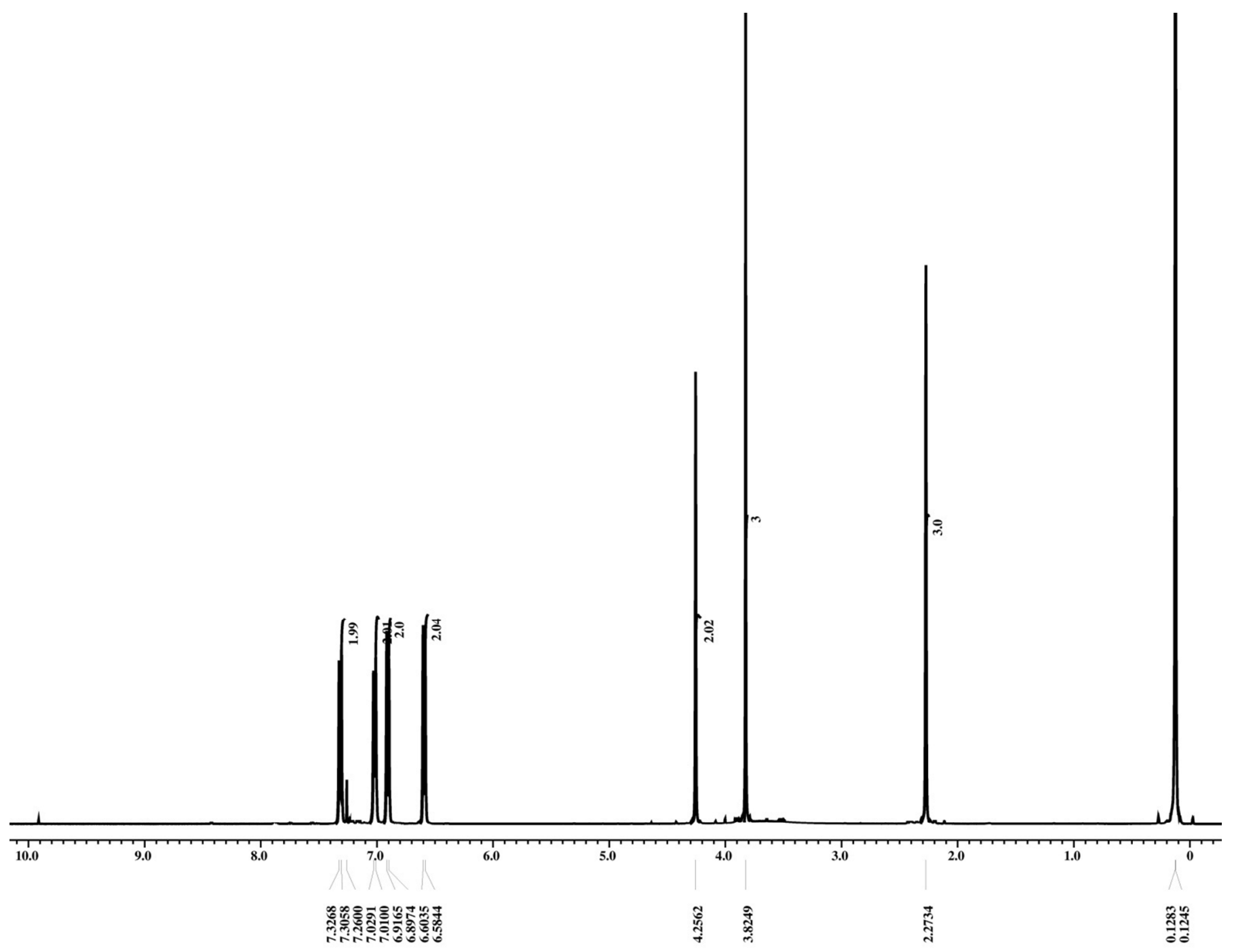




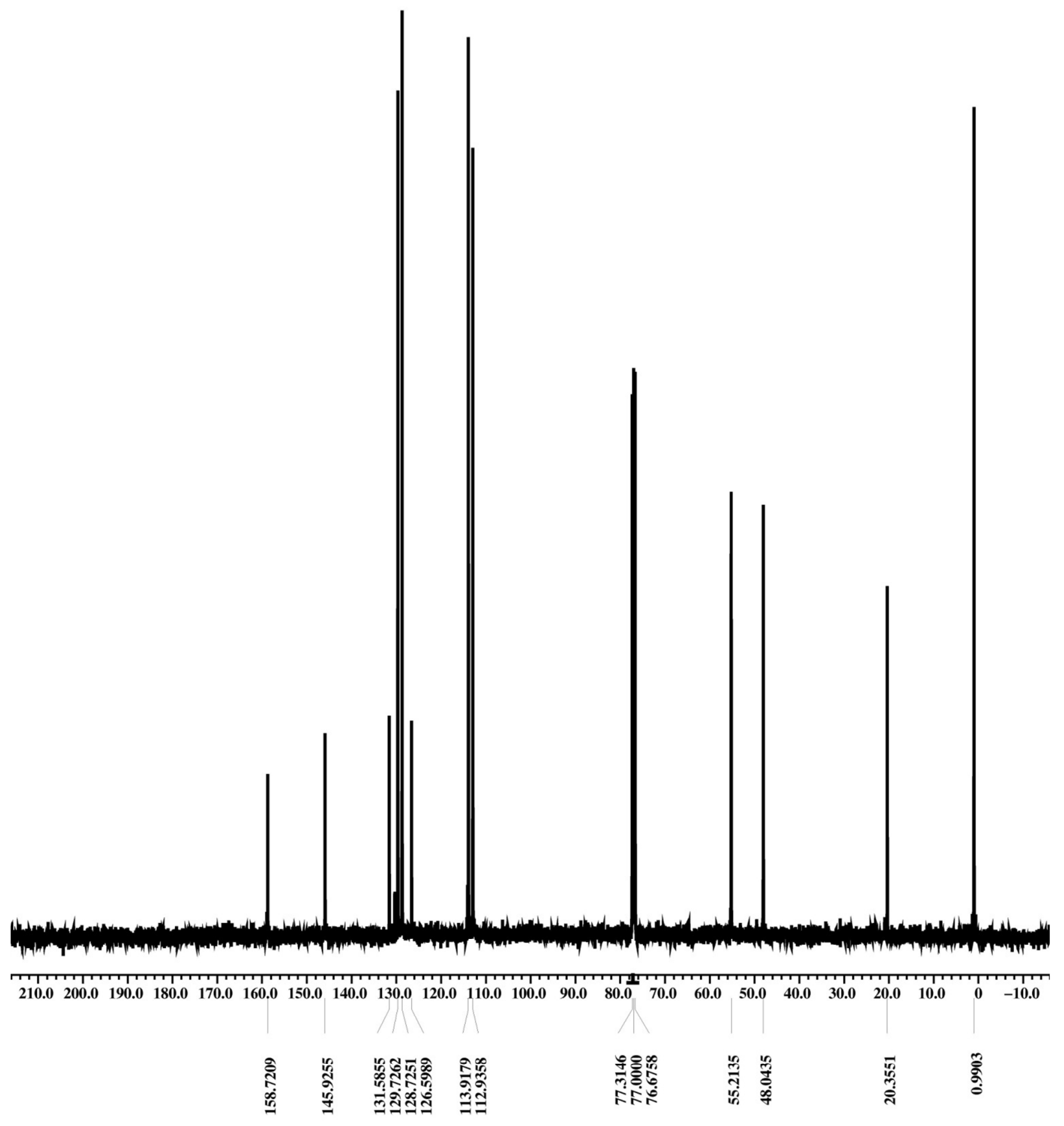


Figure S10. ${ }^{1} \mathrm{H}$ NMR and ${ }^{13} \mathrm{C}$ NMR spectra of N-(4'-(N',N'-Dimethyl)benzyl)-4-methylaniline in $\mathrm{CDCl}_{3}$ (Table 1, Entry 7)<smiles>Cc1ccc(NCc2ccc(N(C)C)cc2)cc1</smiles>

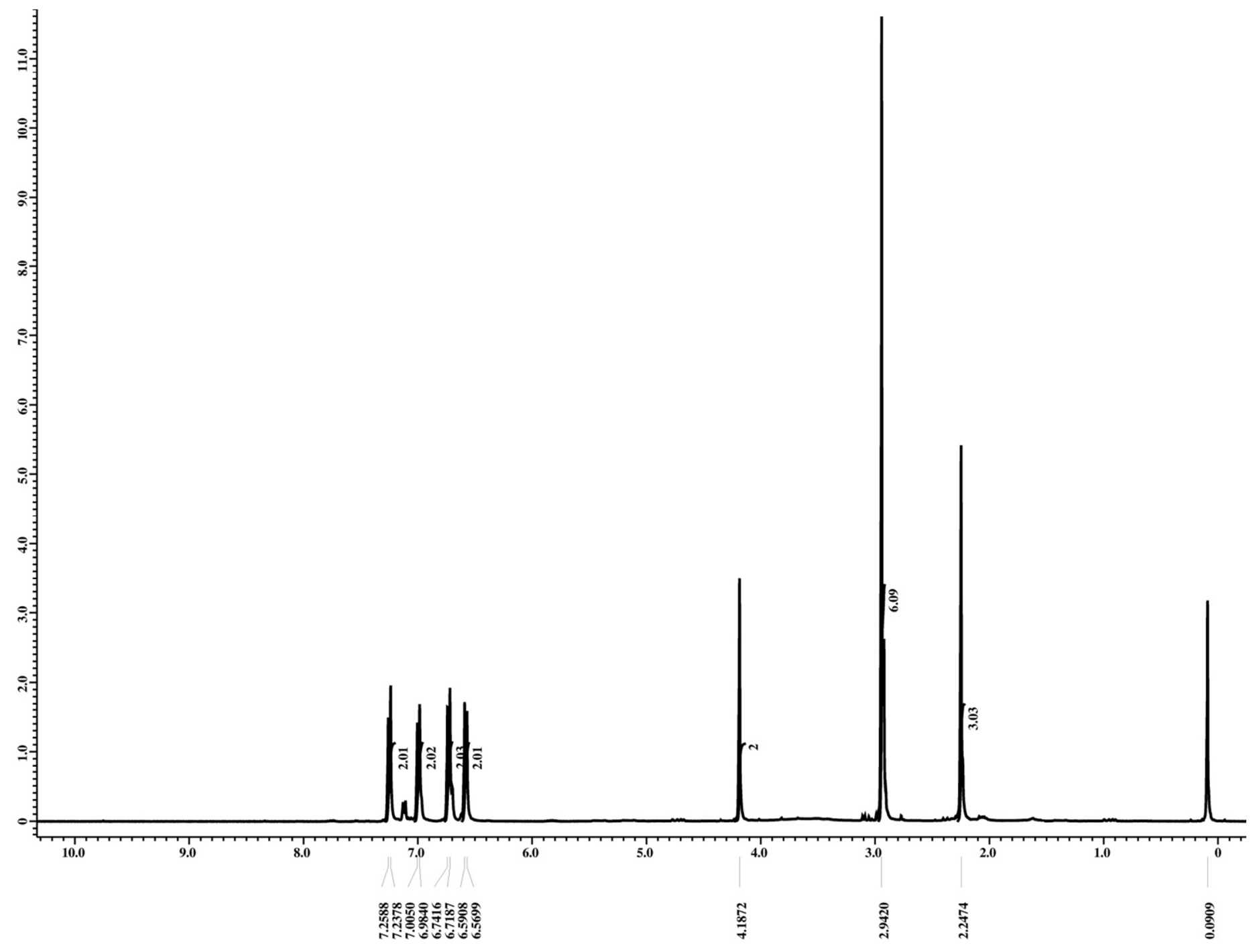

S40 


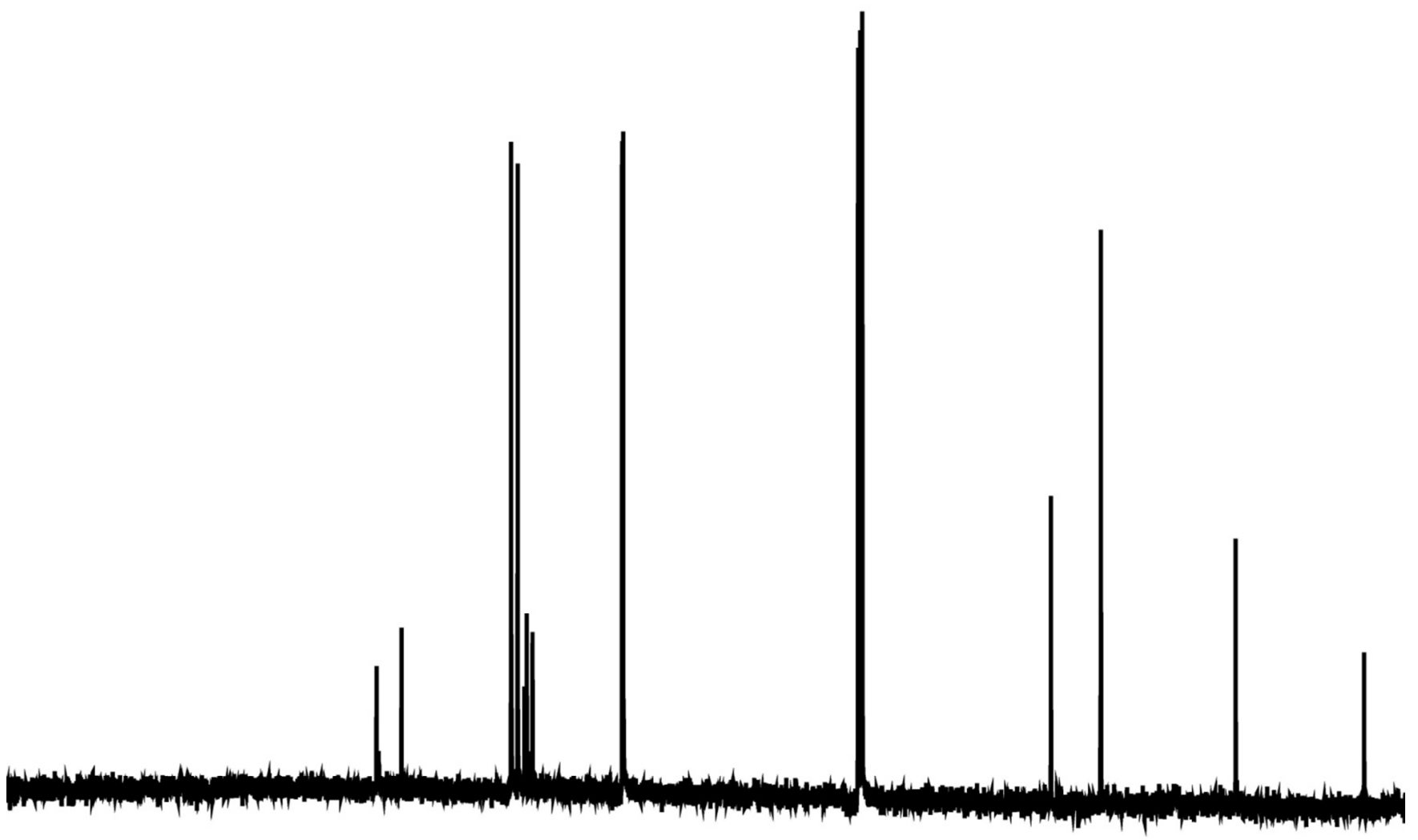

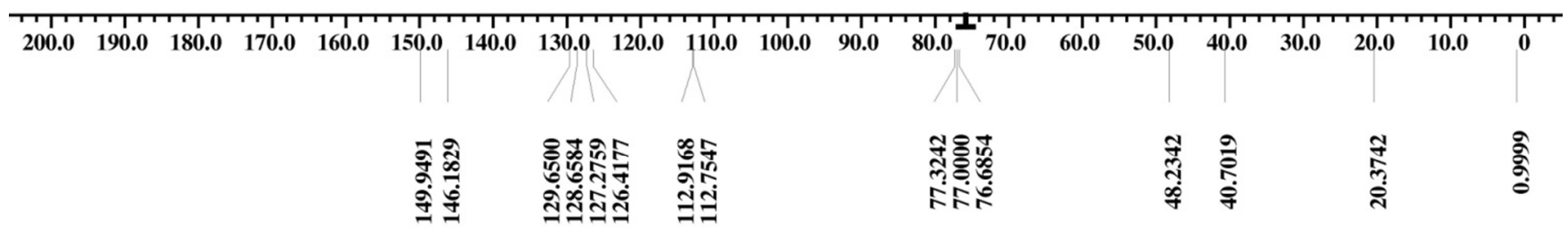


Figure S11. ${ }^{1} \mathrm{H}$ NMR and ${ }^{13} \mathrm{C}$ NMR spectra of $\mathrm{N}$-(4-Iodobenzyl)-4-methylaniline in $\mathrm{CDCl}_{3}$ (Table 1, Entry 8)<smiles>Cc1ccc(NCc2ccc(I)cc2)cc1</smiles>

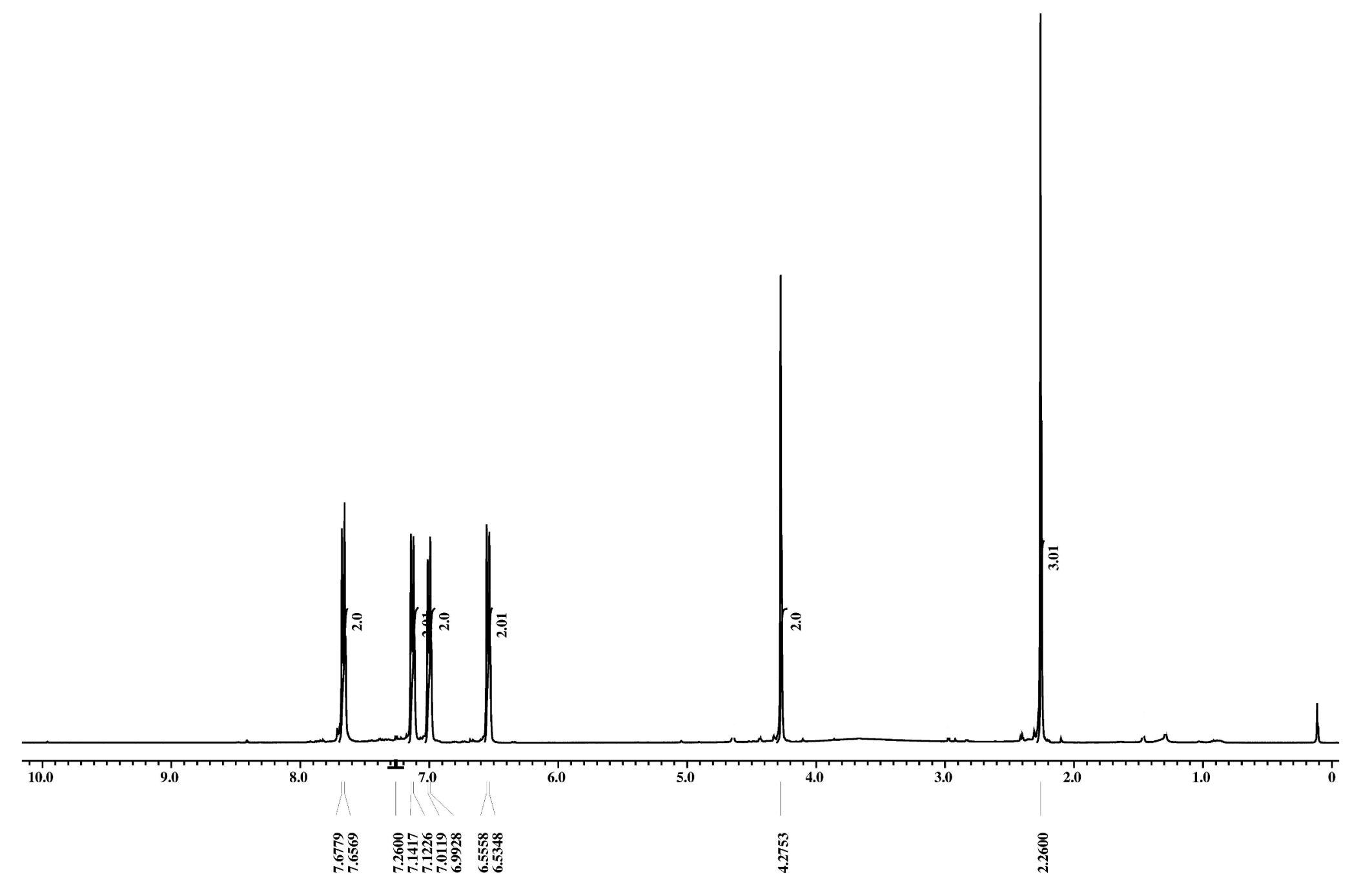




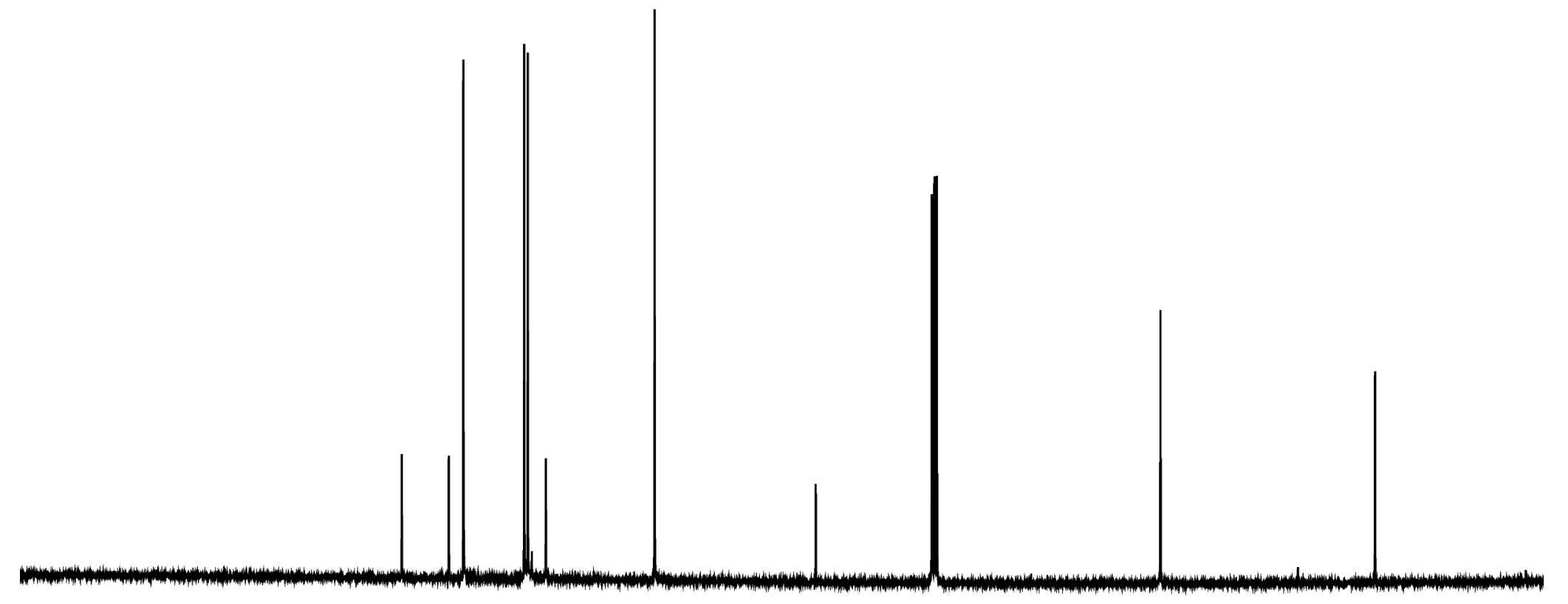

\begin{tabular}{|c|c|c|c|c|c|c|c|c|c|c|c|c|c|c|c|c|c|c|c|}
\hline \multirow[t]{2}{*}{190.0} & 180.0 & 170.0 & 160.0 & 150.0 & 140.0 & 130.0 & 120.0 & 110.0 & 100.0 & 90.0 & 80.0 & 70.0 & 60.0 & 50.0 & 40.0 & 30.0 & 20.0 & 10.0 & 0 \\
\hline & & & & & 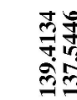 & 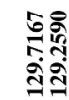 & & 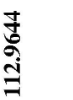 & & 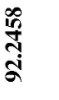 & & & & $\begin{array}{l}8 \\
\sigma \\
\sigma\end{array}$ & & & 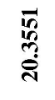 & & \\
\hline
\end{tabular}


Figure S12. ${ }^{1} \mathrm{H}$ NMR and ${ }^{13} \mathrm{C}$ NMR spectra of N-(4-Bromobenzyl)-4-methylaniline in $\mathrm{CDCl}_{3}$ (Table 1, Entry 9)
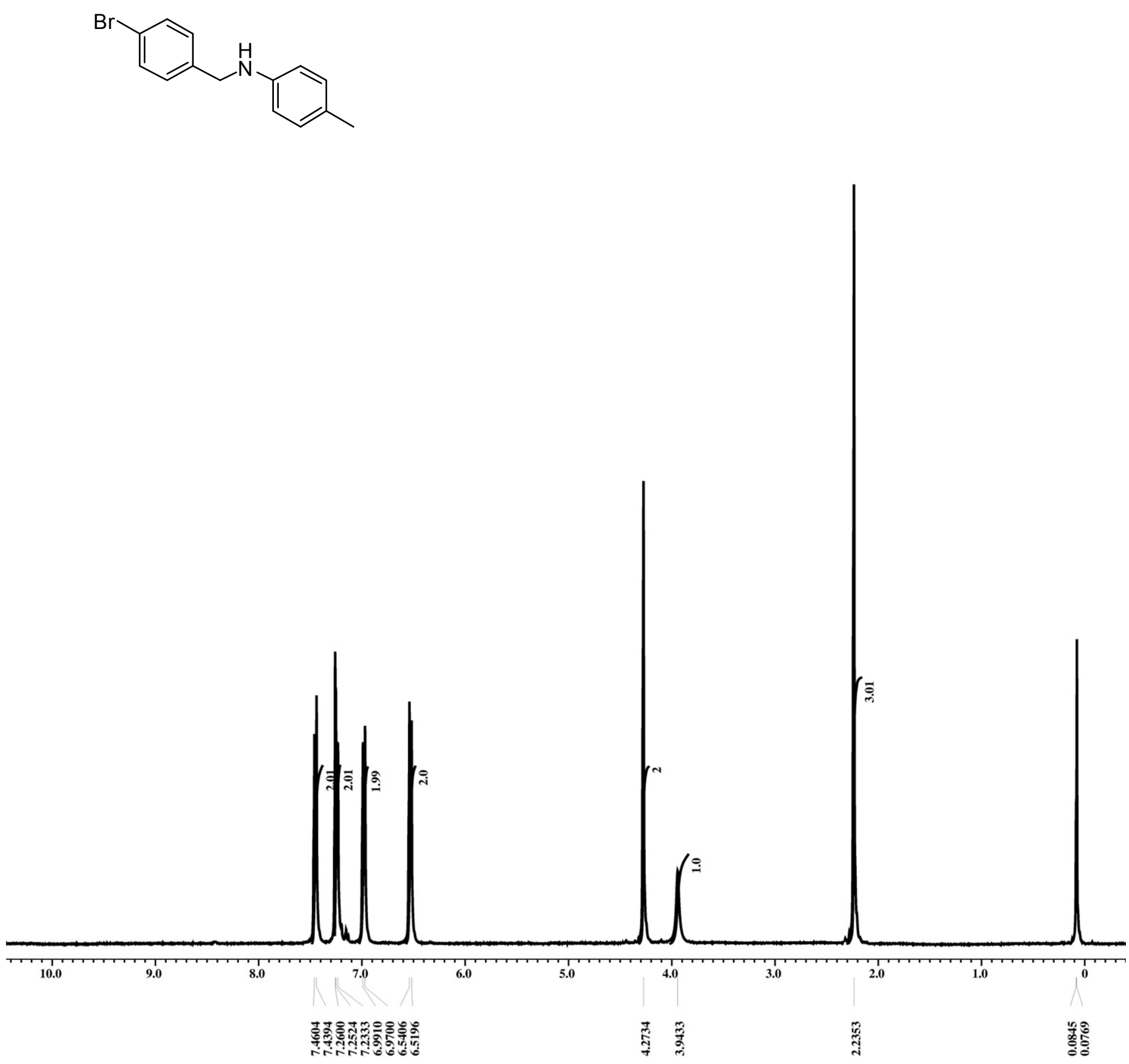


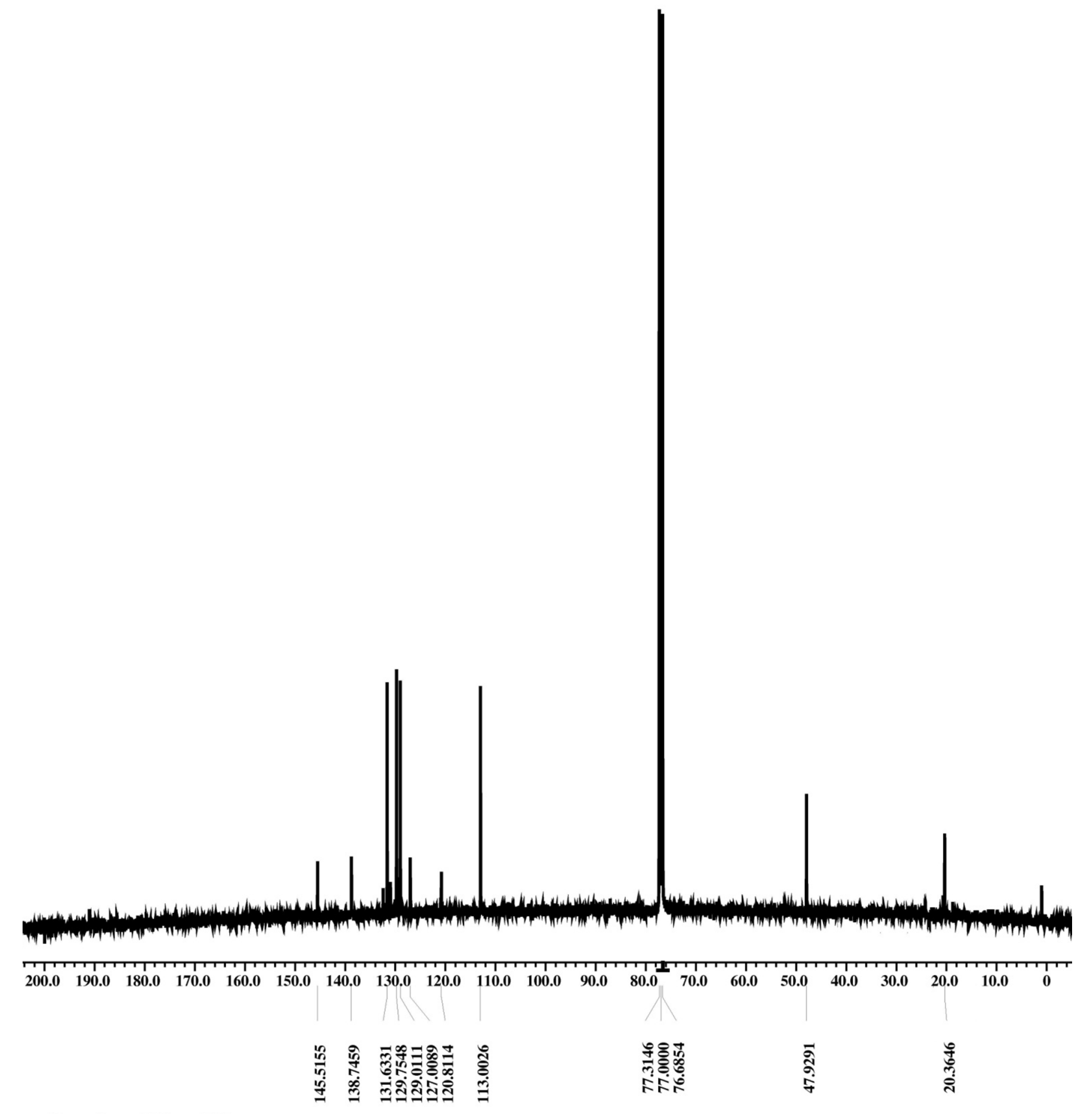


Figure S13. ${ }^{1} \mathrm{H}$ NMR and ${ }^{13} \mathrm{C}$ NMR spectra of N-(4-Chlorobenzyl)-4-methylaniline in $\mathrm{CDCl}_{3}$ (Table 1, Entry 10)<smiles>Cc1ccc(NCc2ccc(Cl)cc2)cc1</smiles>

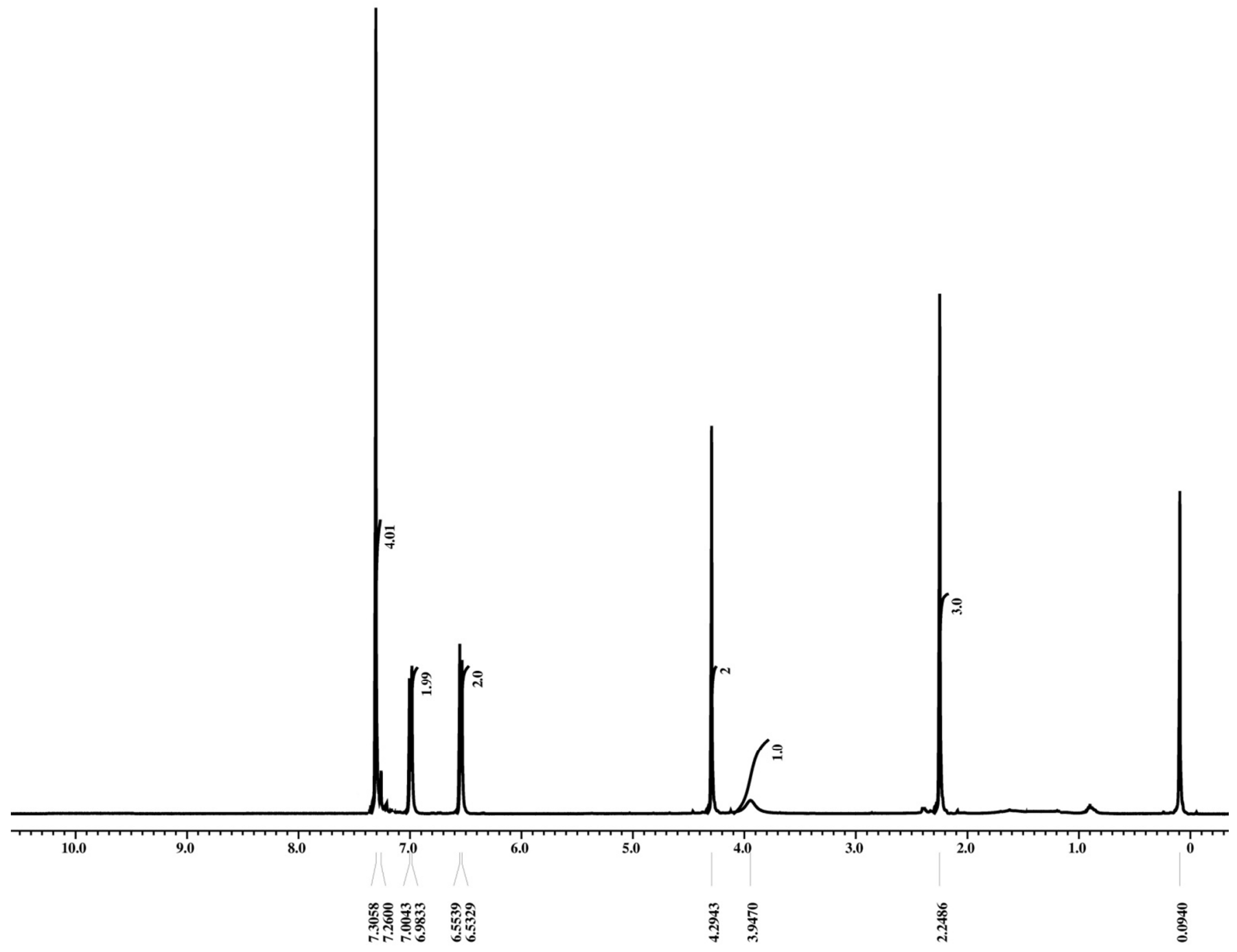




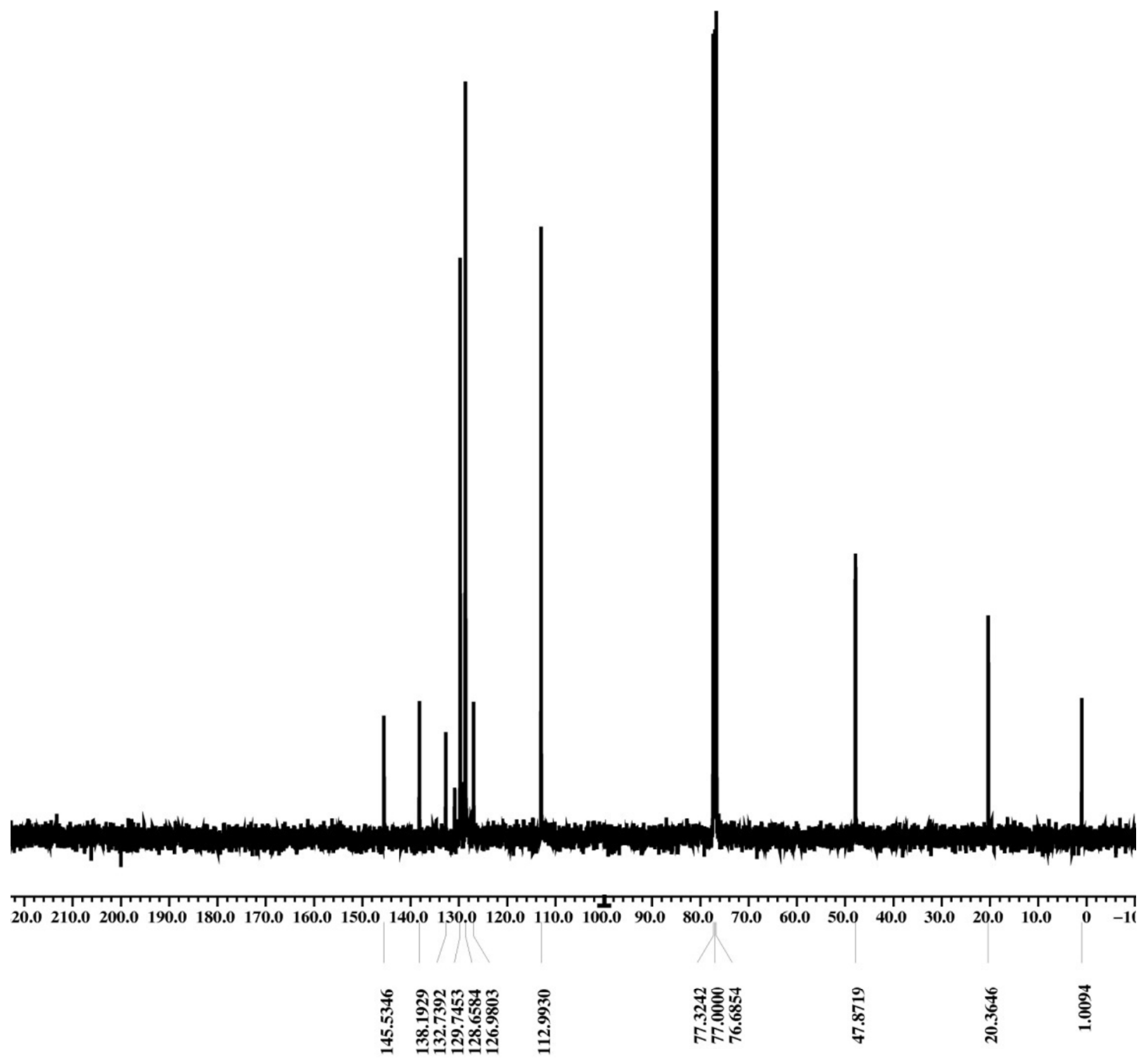




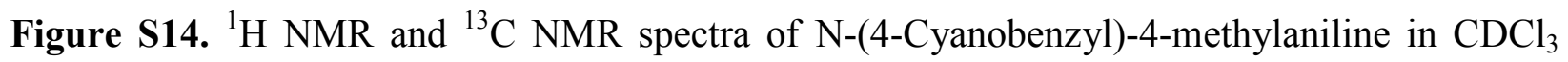
(Table 1, Entry 11)<smiles>Cc1ccc(NCc2ccc(C#N)cc2)cc1</smiles>
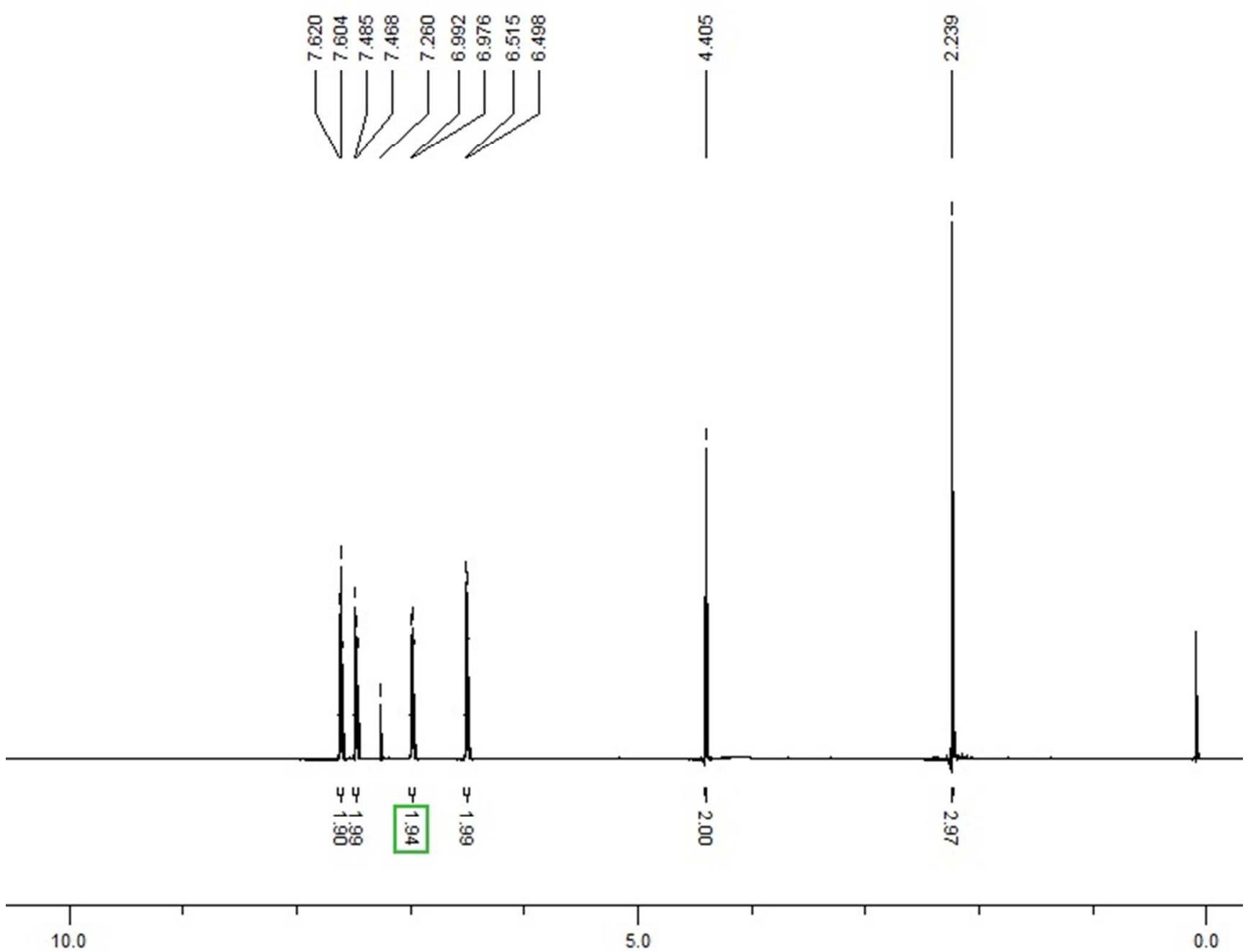

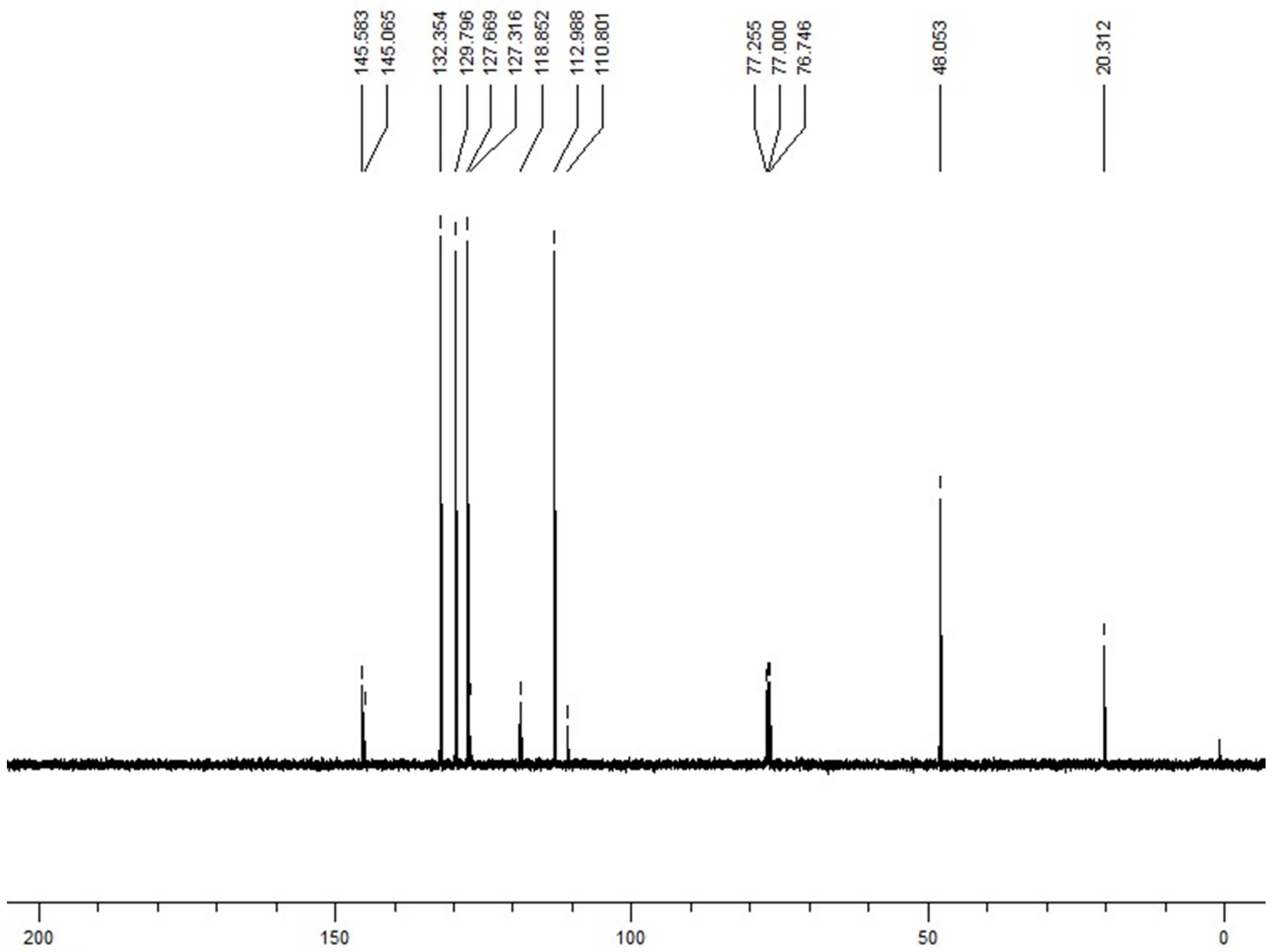
Figure S15. ${ }^{1} \mathrm{H}$ NMR and ${ }^{13} \mathrm{C}$ NMR spectra of 4-Methyl-N-(4-nitrobenzyl)aniline in $\mathrm{CDCl}_{3}$ (Table 1, Entry 12)<smiles>Cc1ccc(NCc2ccc([N+](=O)[O-])cc2)cc1</smiles>

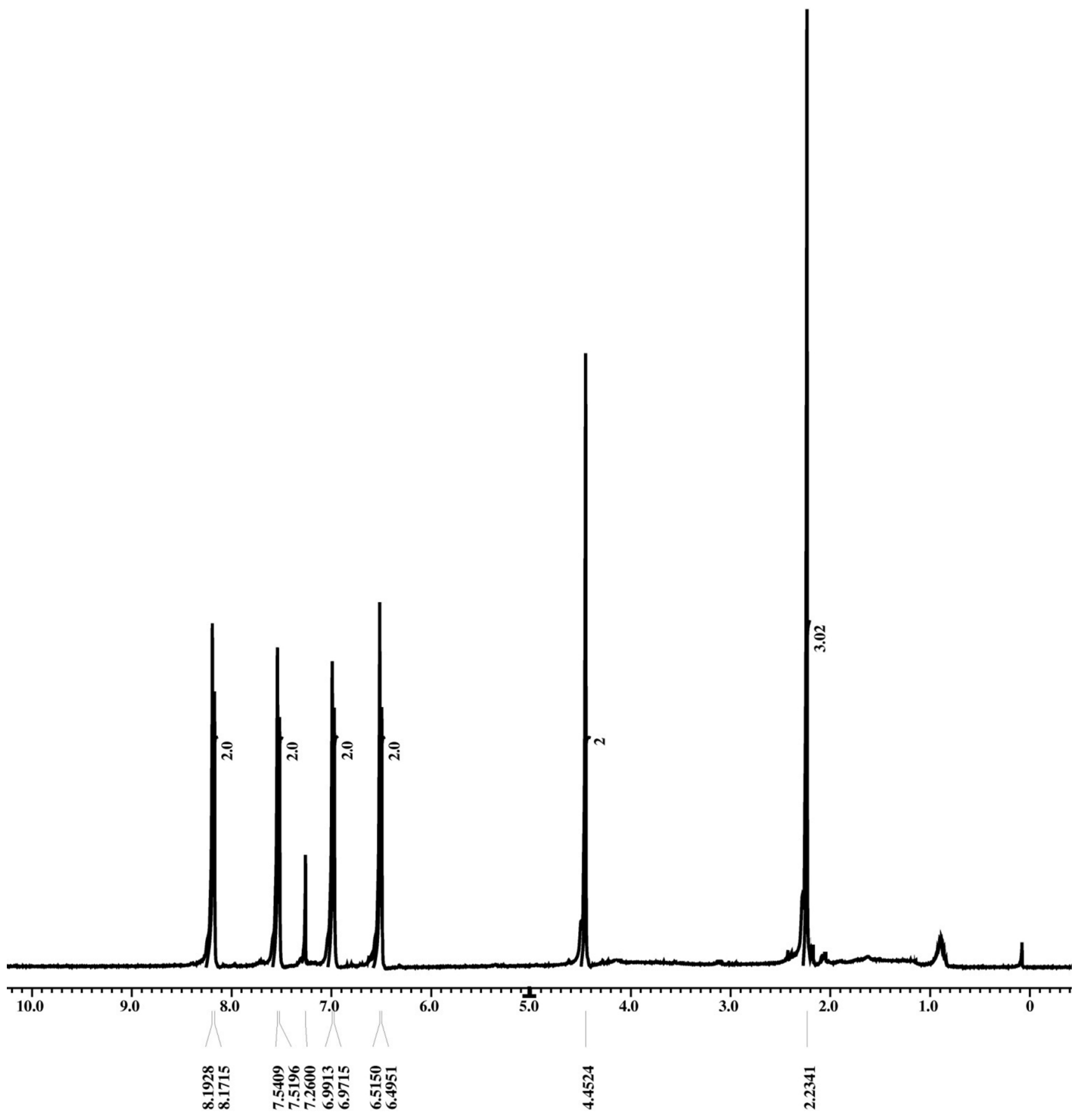




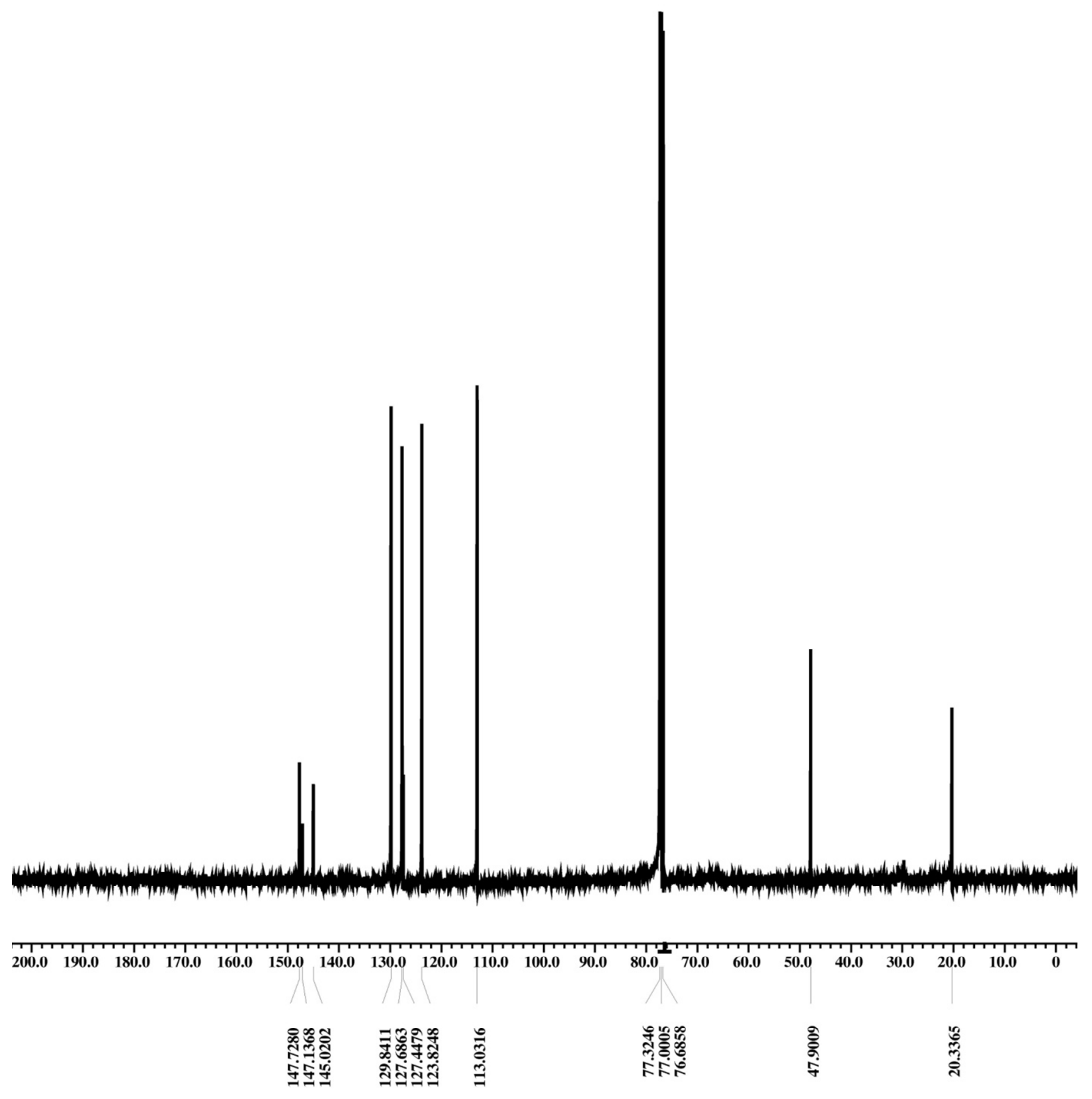


Figure S16. ${ }^{1} \mathrm{H} \quad \mathrm{NMR}$ and ${ }^{13} \mathrm{C}$ NMR spectra of N-[4-(Methyloxycarbonyl)benzyl]-4-<smiles>COC(=O)c1ccc(CNc2ccc(C)cc2)cc1</smiles>
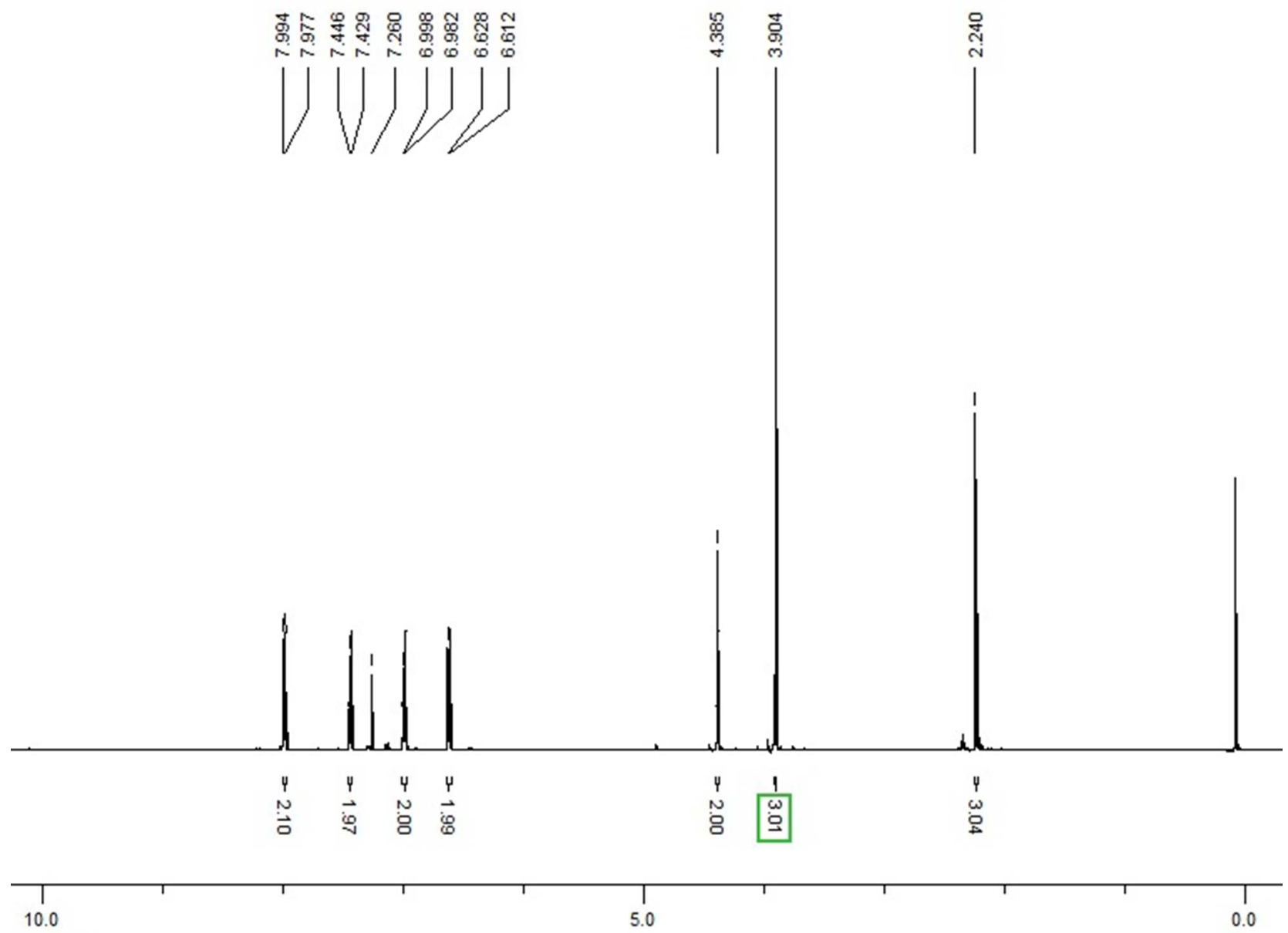

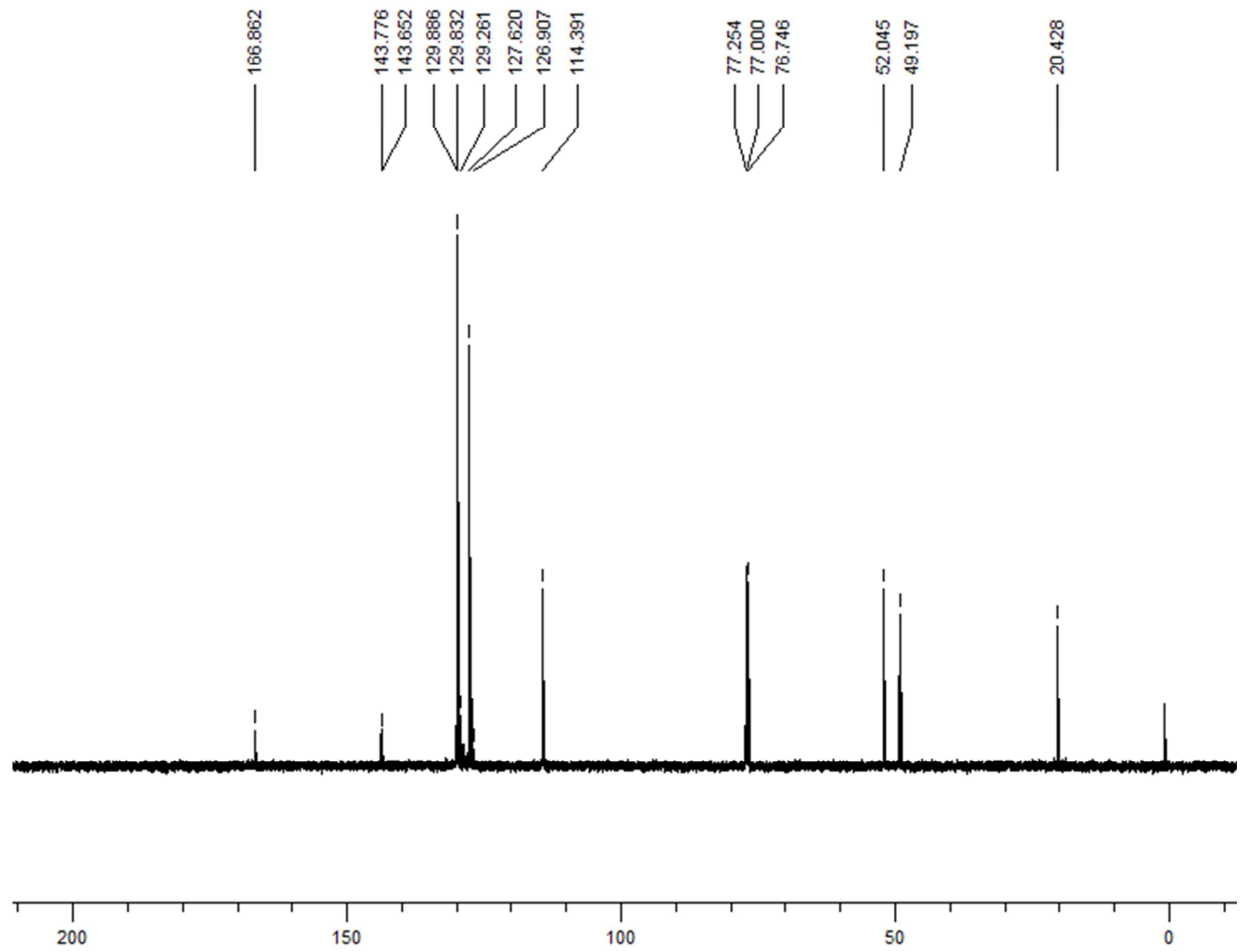
Figure S17. ${ }^{1} \mathrm{H}$ NMR and ${ }^{13} \mathrm{C}$ NMR spectra of $\mathrm{N}-\left[(\right.$ Ferrocenyl)methyl $] 4-$ methylaniline in $\mathrm{CDCl}_{3}$ (Table 1, Entry 15)<smiles></smiles>

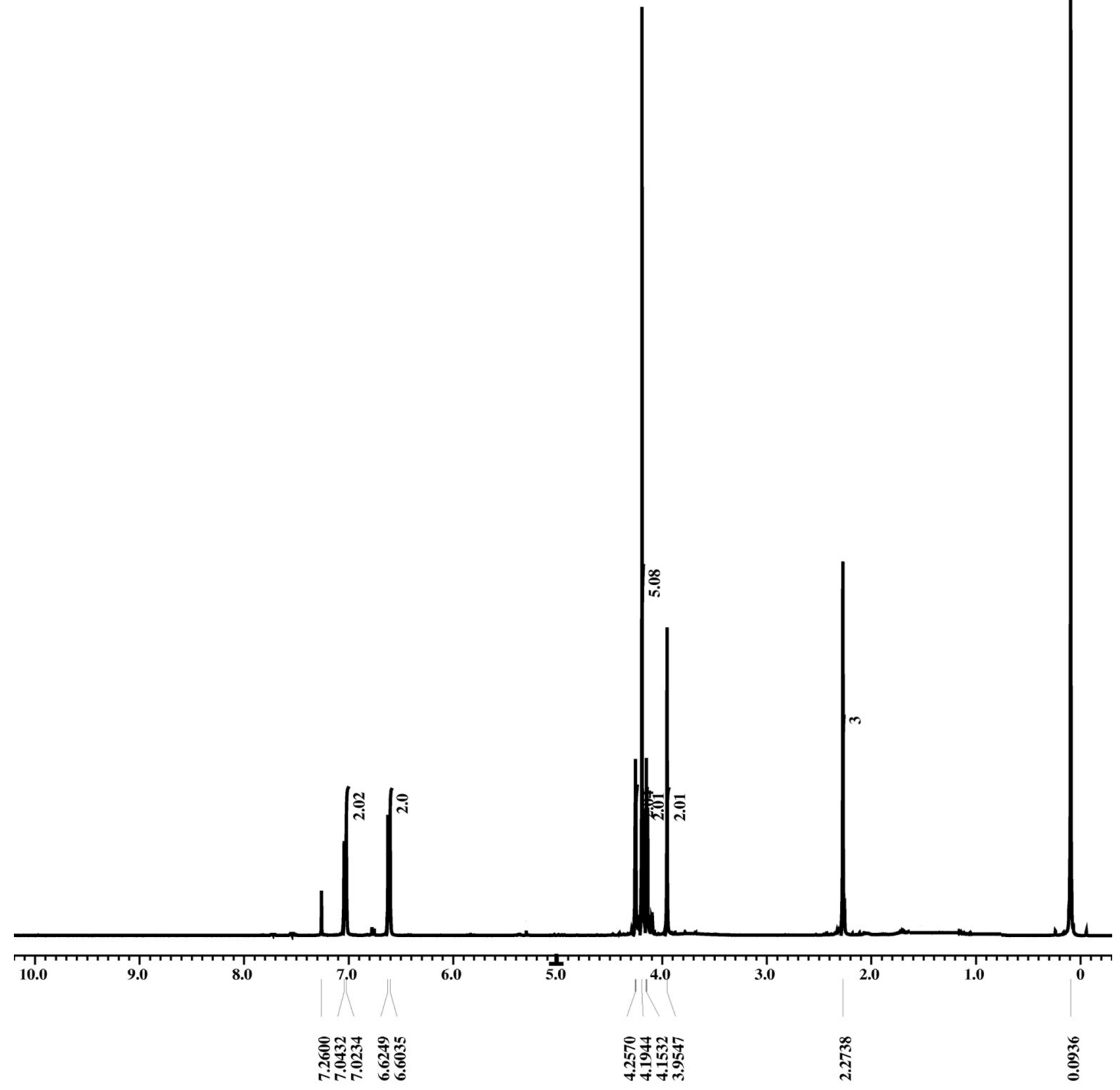




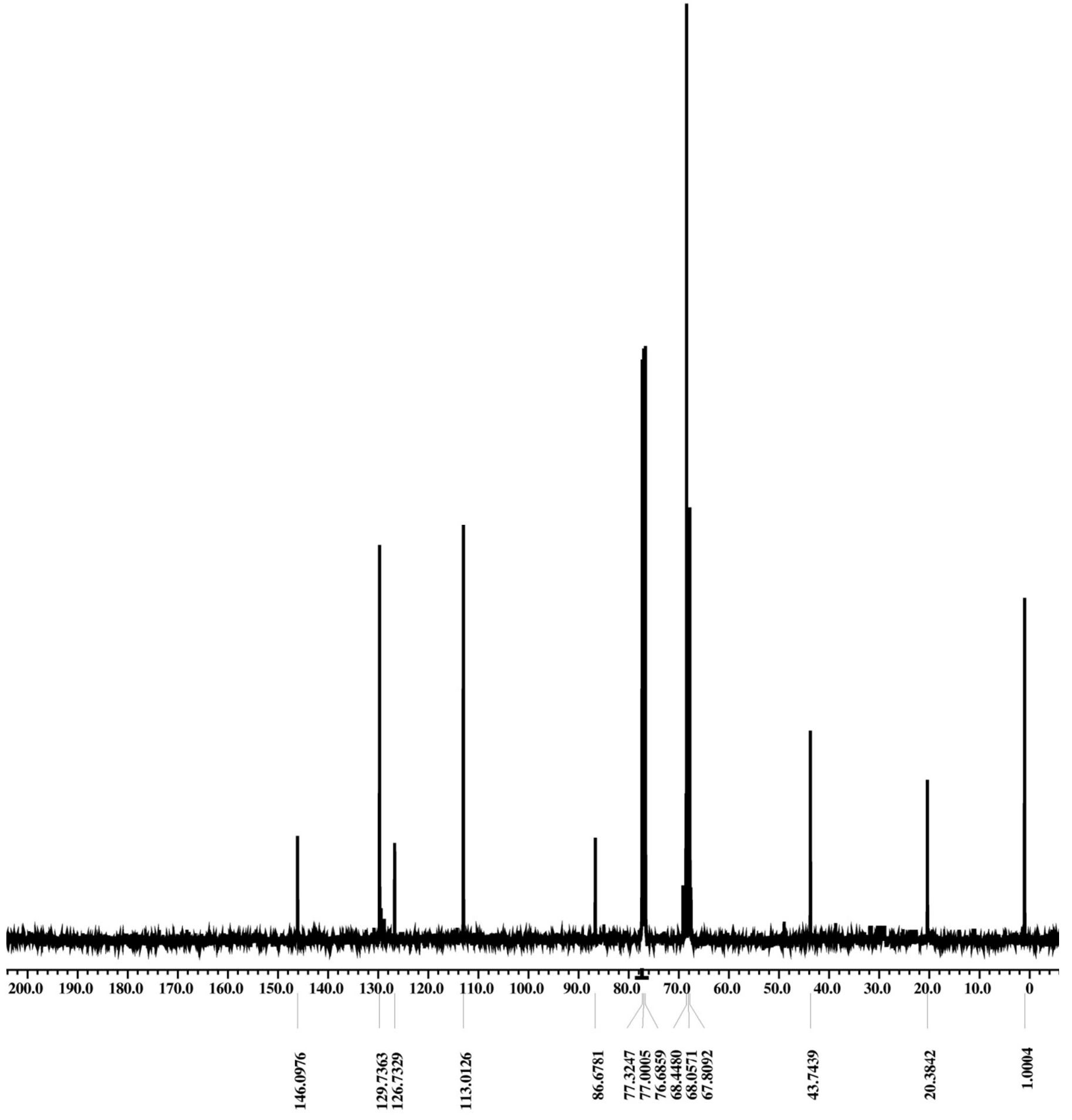


Figure S18. ${ }^{1} \mathrm{H}$ NMR and ${ }^{13} \mathrm{C}$ NMR spectra of N-(4-Cyanobenzyl)-4-methoxylaniline in $\mathrm{CDCl}_{3}$ (Table 1, Entry 16)<smiles>COc1ccc(NCc2ccc(C#N)cc2)cc1</smiles>
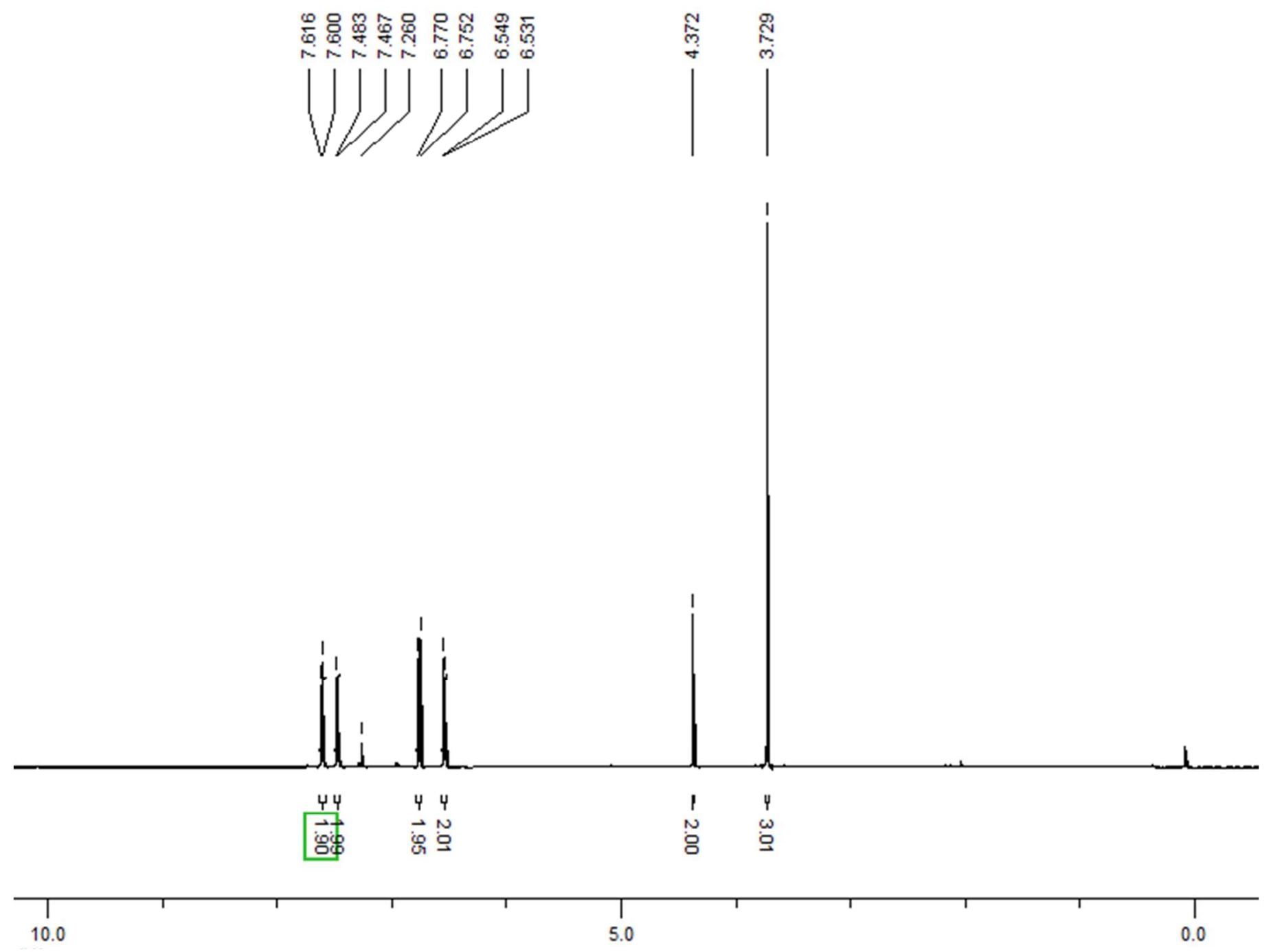

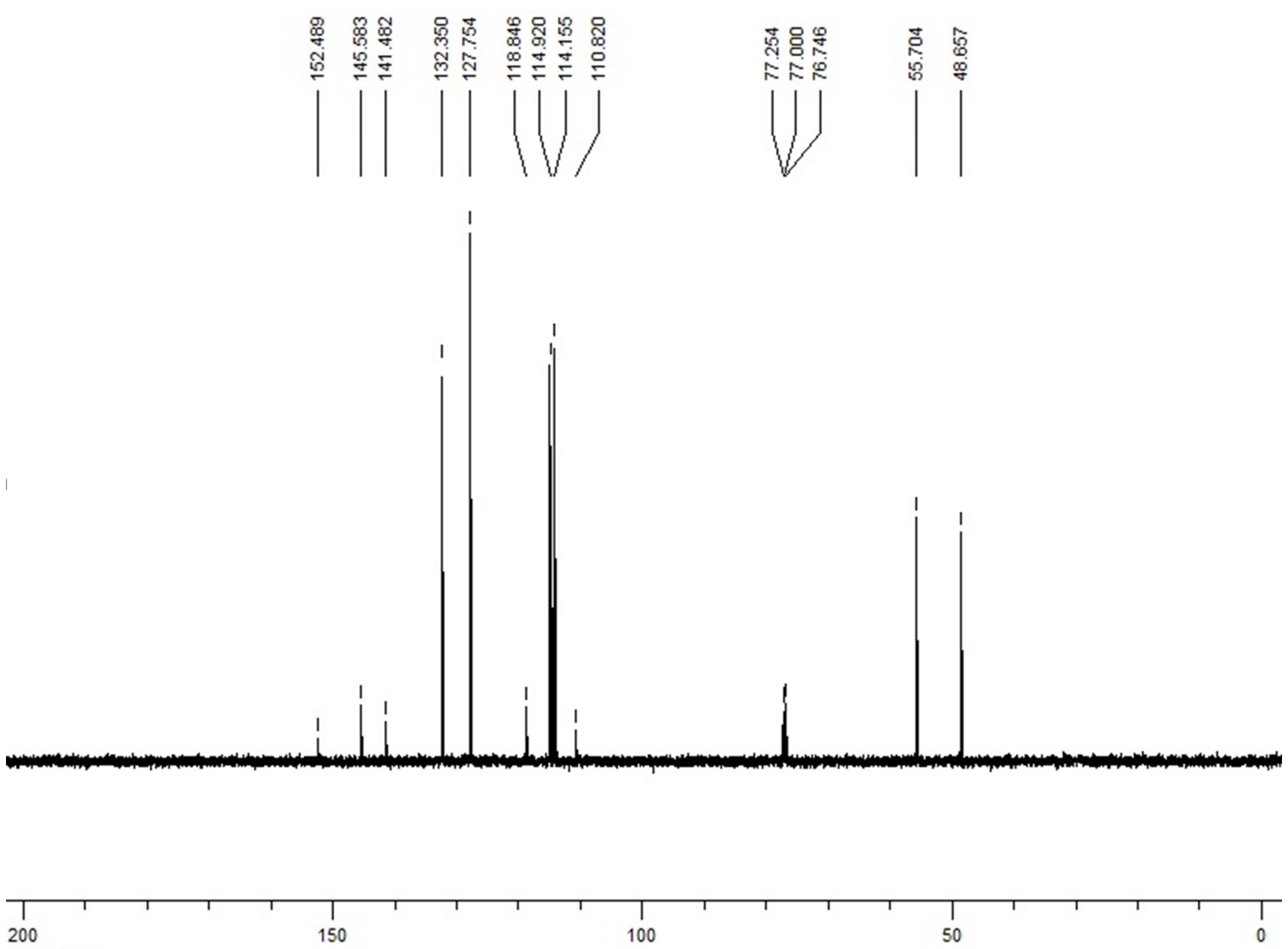
Figure S19. ${ }^{1} \mathrm{H}$ NMR and ${ }^{13} \mathrm{C}$ NMR spectra of N-(4-nitrobenzyl)-4-Methoxylaniline in $\mathrm{CDCl}_{3}$ (Table 1, Entry 17)<smiles>COc1ccc(NCc2ccc([N+](=O)[O-])cc2)cc1</smiles>
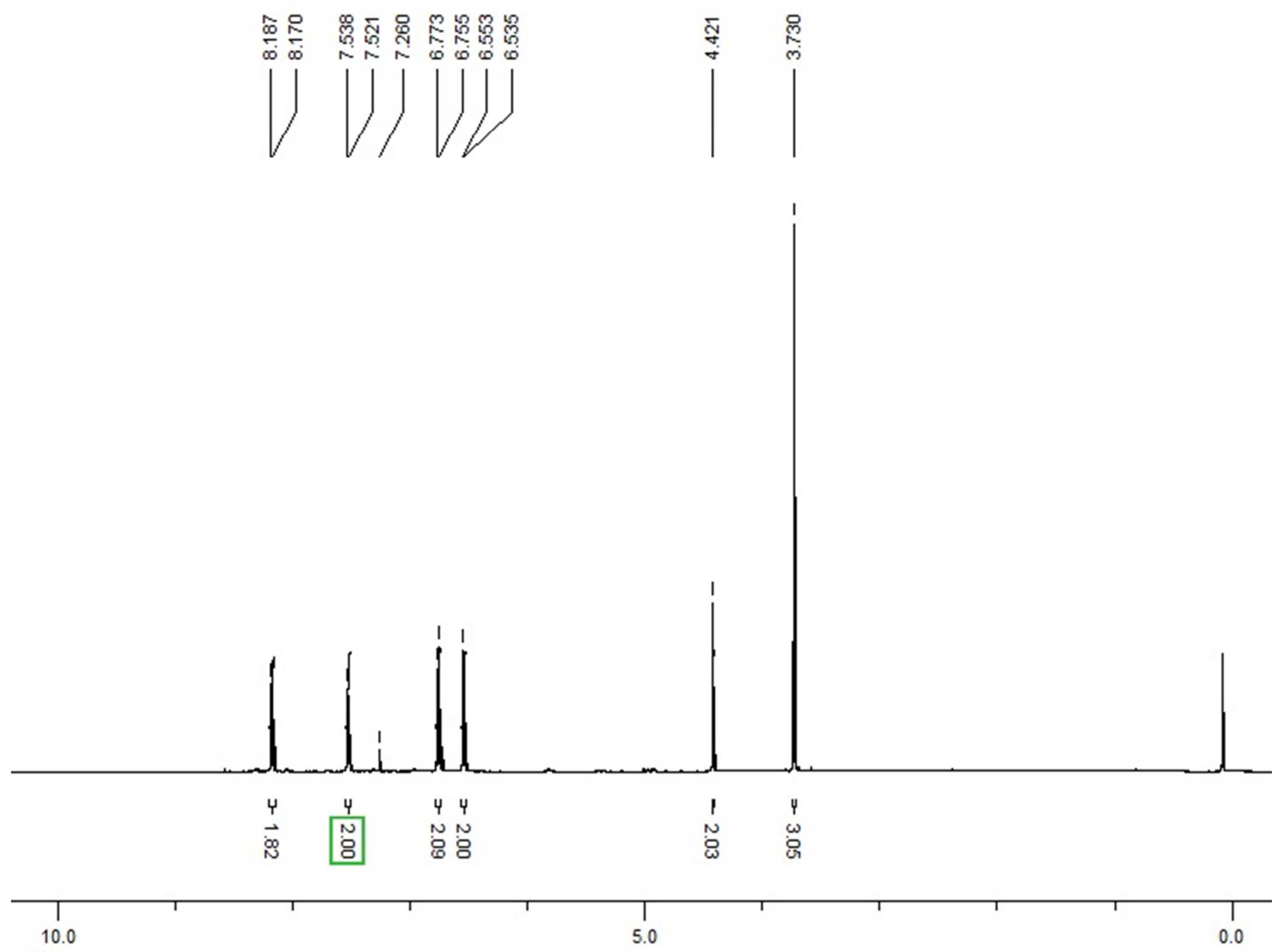

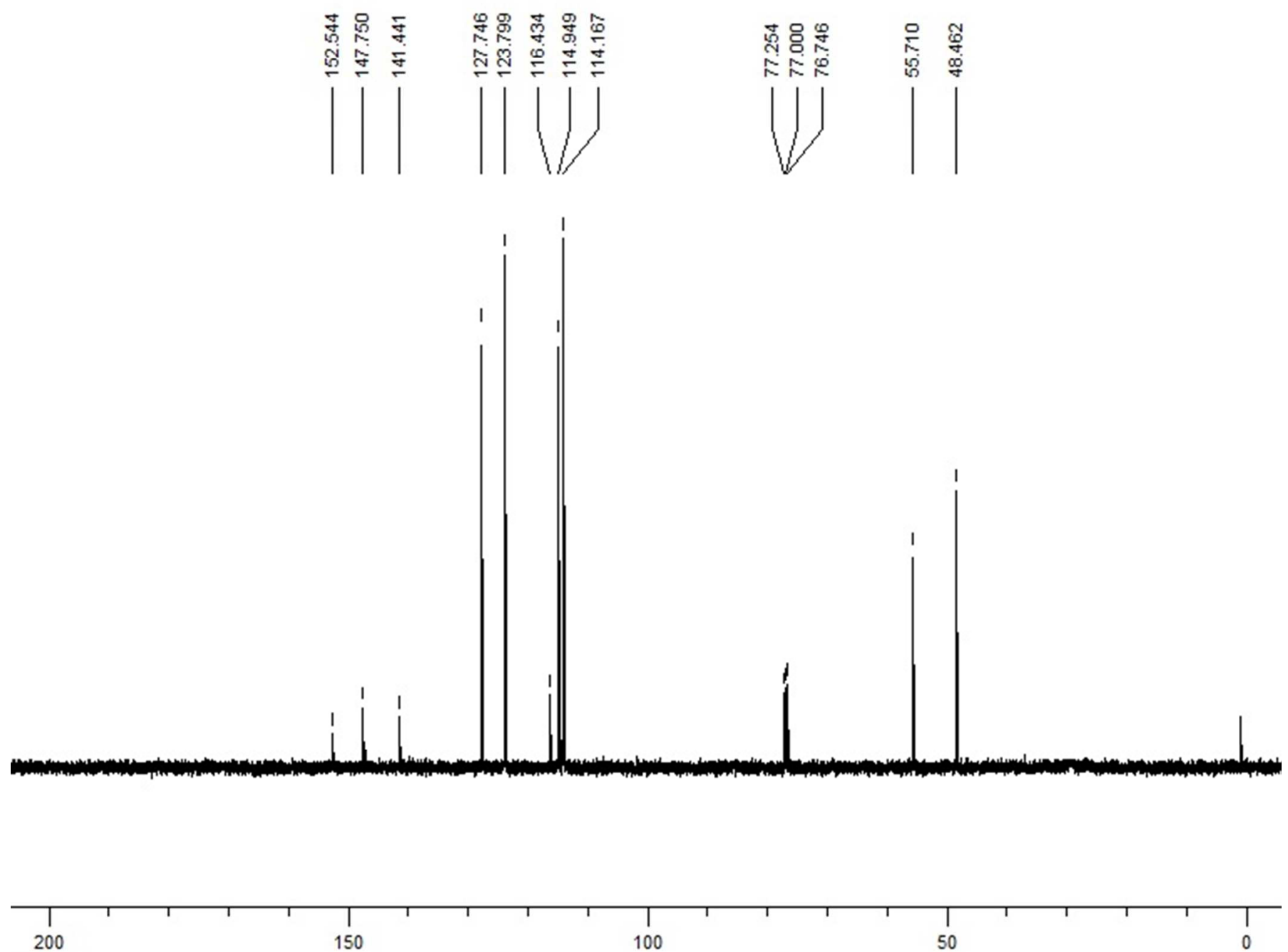
Figure S20. ${ }^{1} \mathrm{H}$ NMR and ${ }^{13} \mathrm{C}$ NMR spectra of 4-Methoxy-N-(3,4,5-trimethoxybenzyl)aniline in $\mathrm{CDCl}_{3}$ (Table 1, Entry 18)<smiles>COc1ccc(NCc2cc(OC)c(OC)c(OC)c2)cc1</smiles>

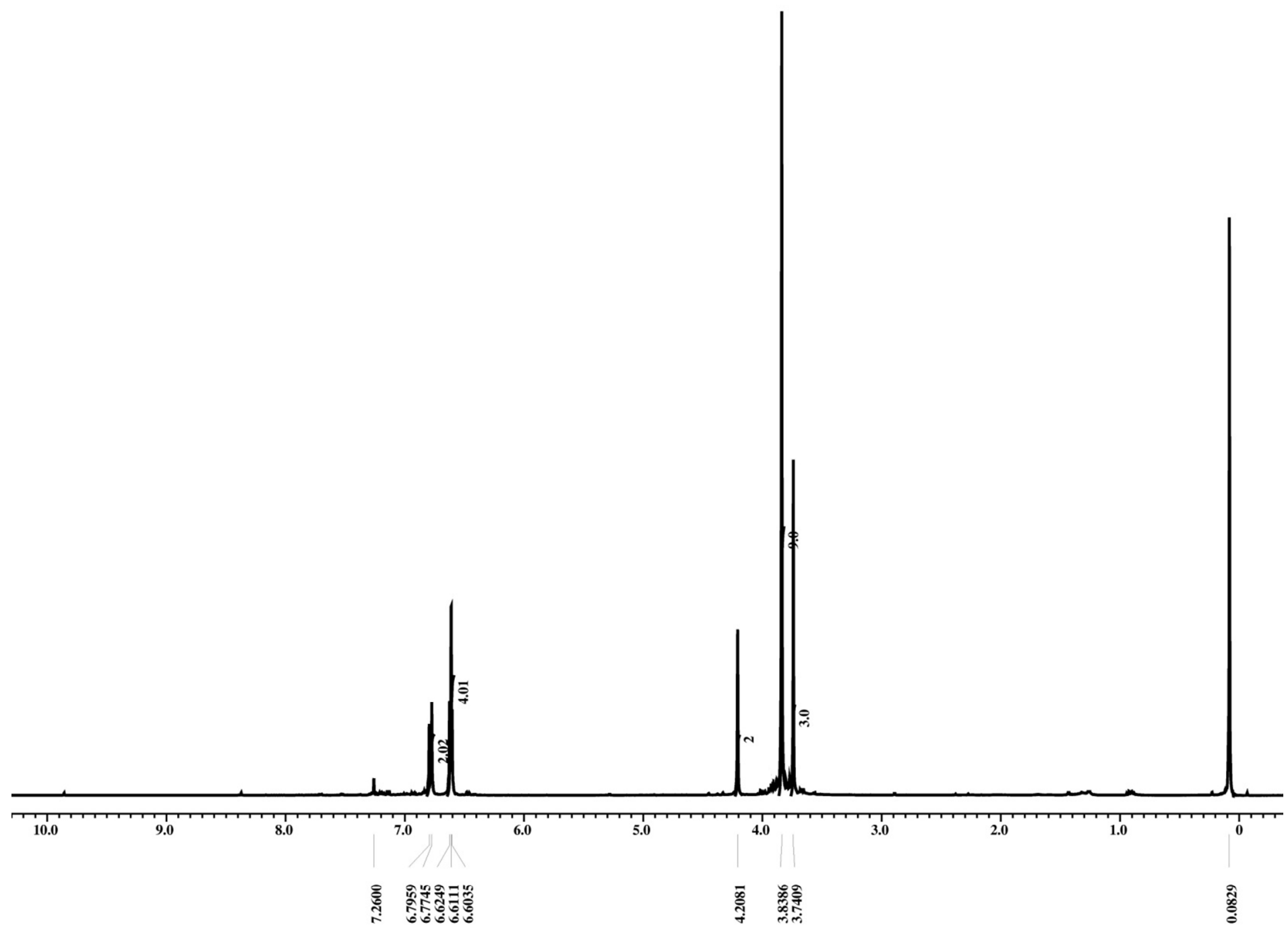




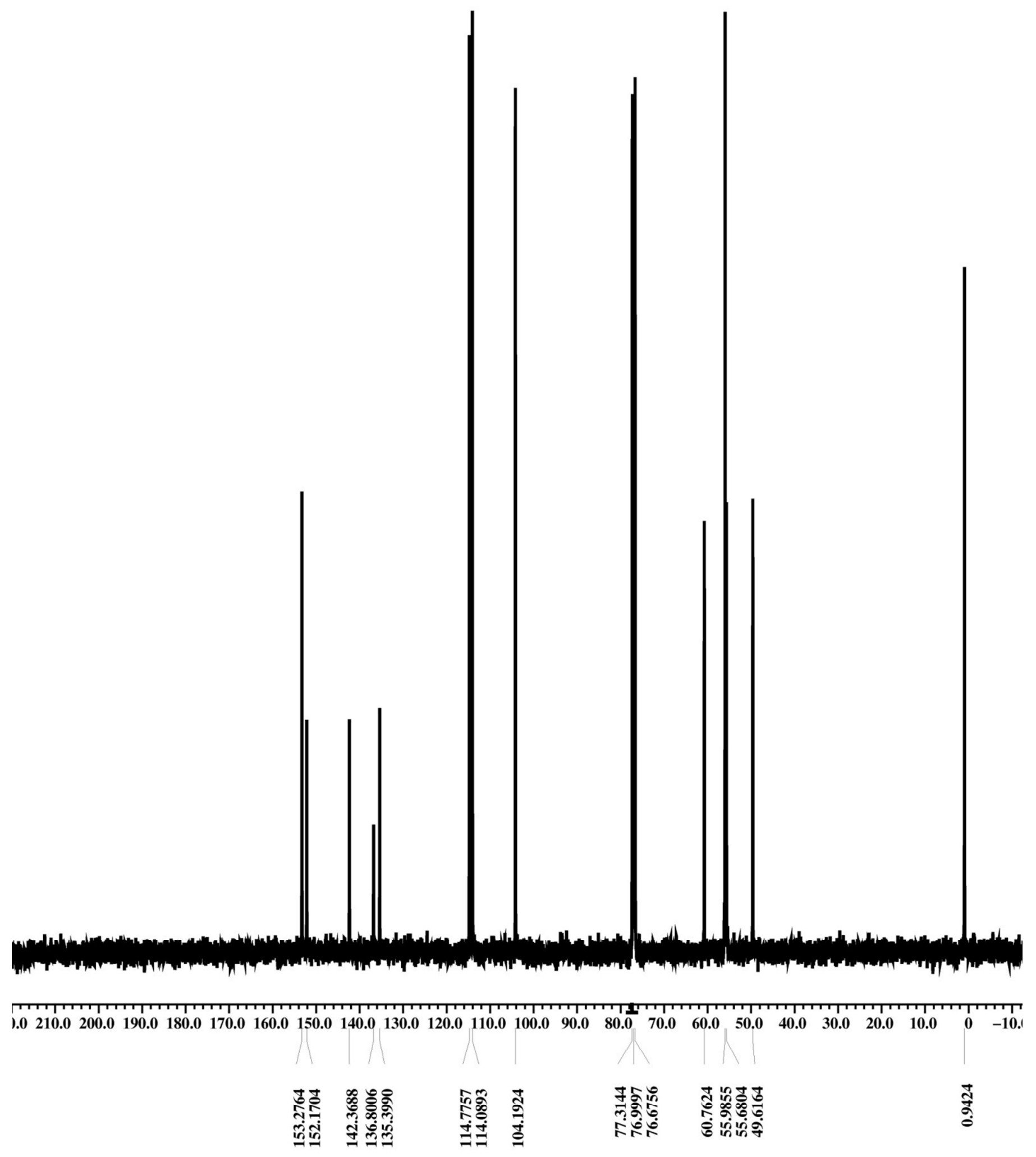


Figure S21. ${ }^{1} \mathrm{H}$ NMR and ${ }^{13} \mathrm{C}$ NMR spectra of $2-\{$ (phenylamino)methyl $\}$ phenol in $\mathrm{CDCl}_{3}$ (Table 1, Entry 19)<smiles>Oc1ccccc1CNc1ccccc1</smiles>
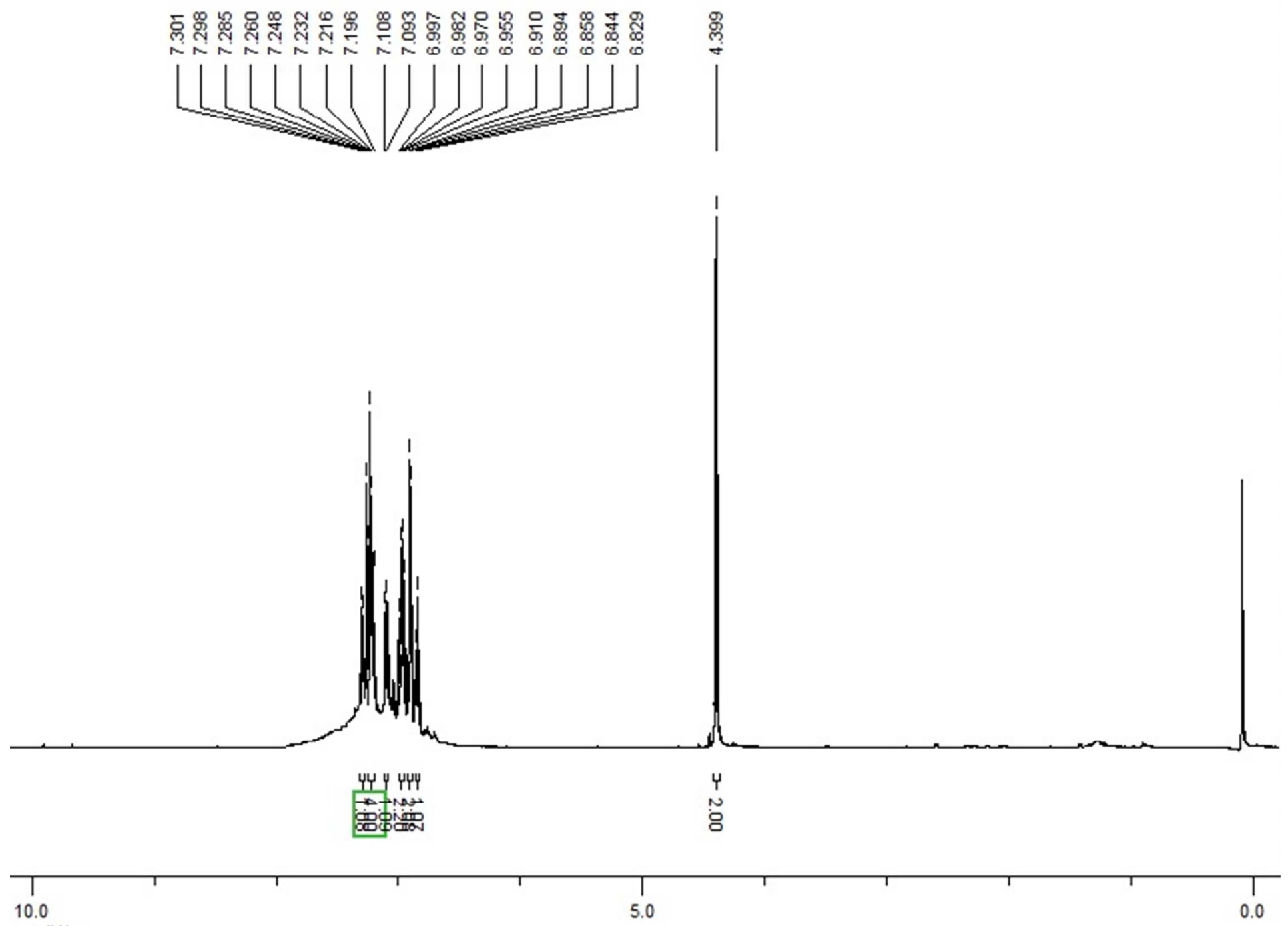


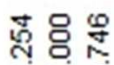

会的

产

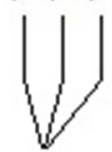

g

i
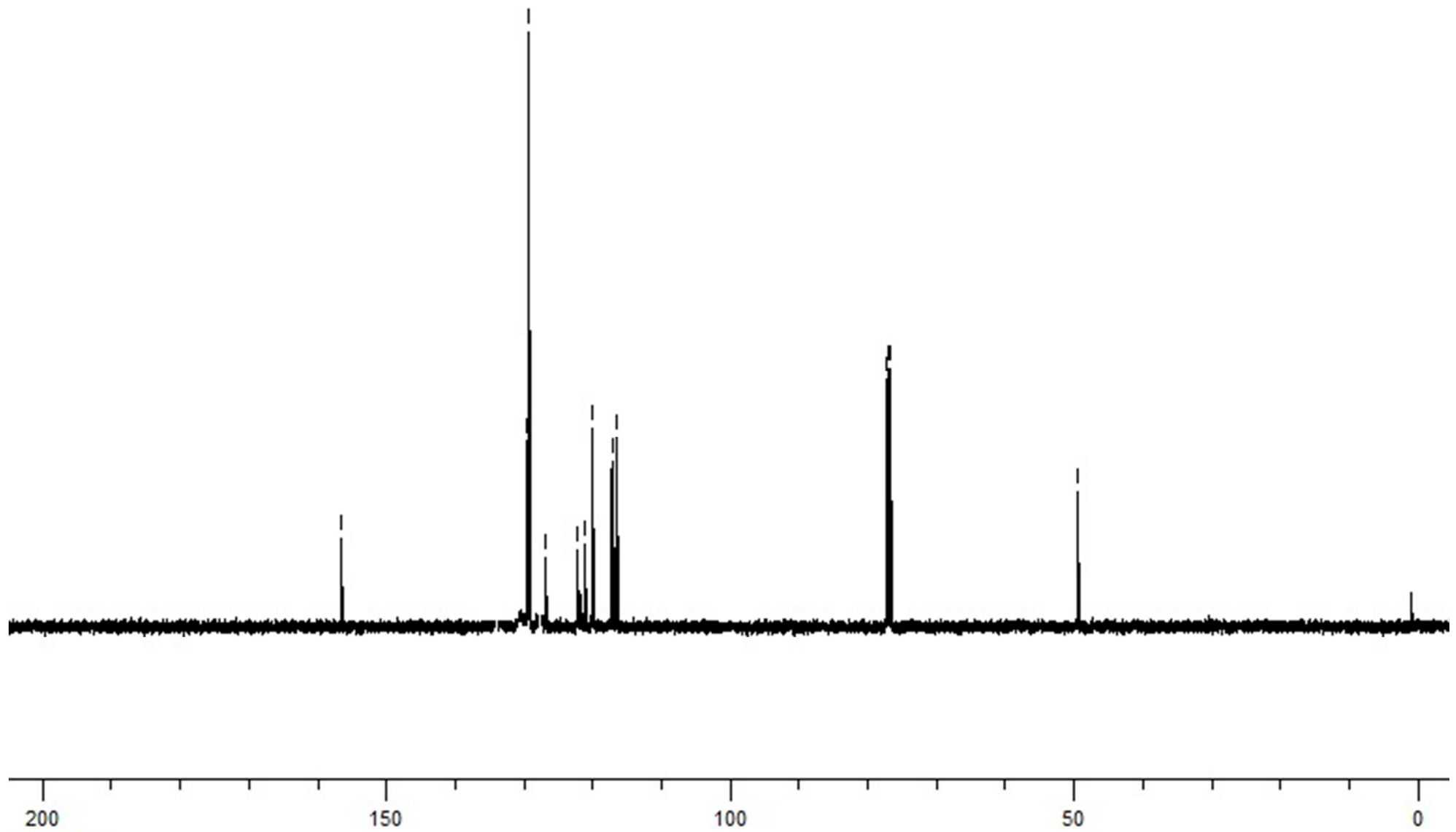
Figure S22. ${ }^{1} \mathrm{H}$ NMR and ${ }^{13} \mathrm{C}$ NMR spectra of 4-(2-hydroxybenzylamino)benzoic acid in $\mathrm{CDCl}_{3}$ (Table 1, Entry 20)<smiles>O=C(O)c1ccc(NCc2ccccc2O)cc1</smiles>
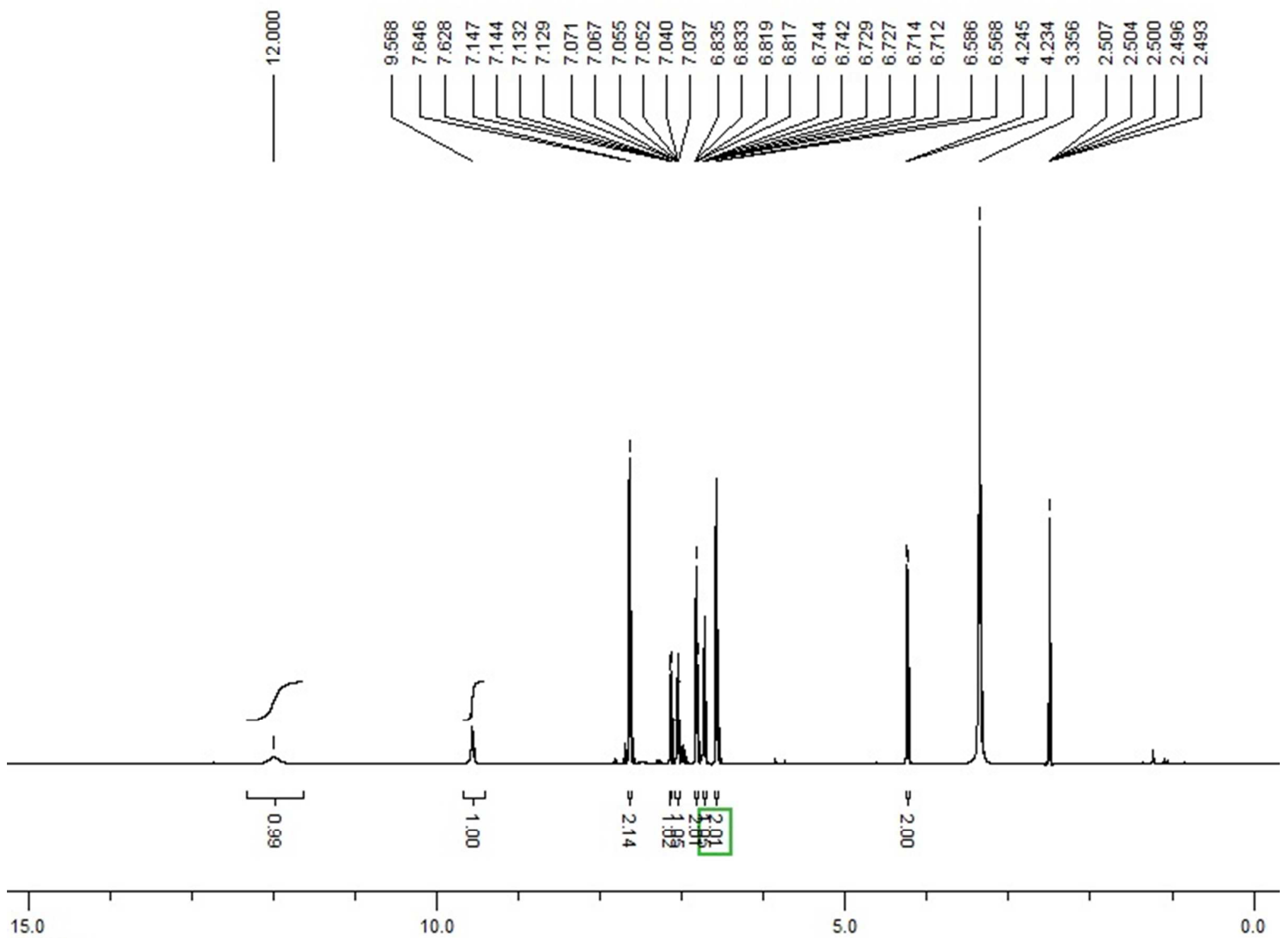


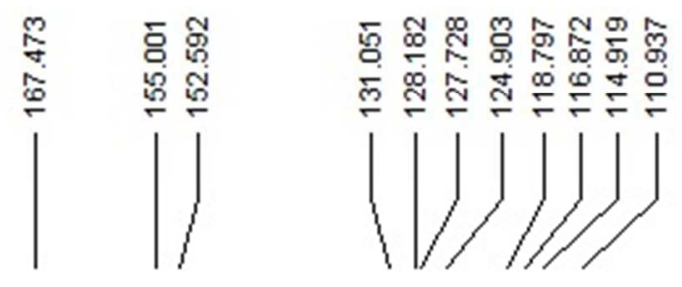

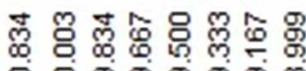

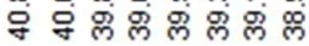
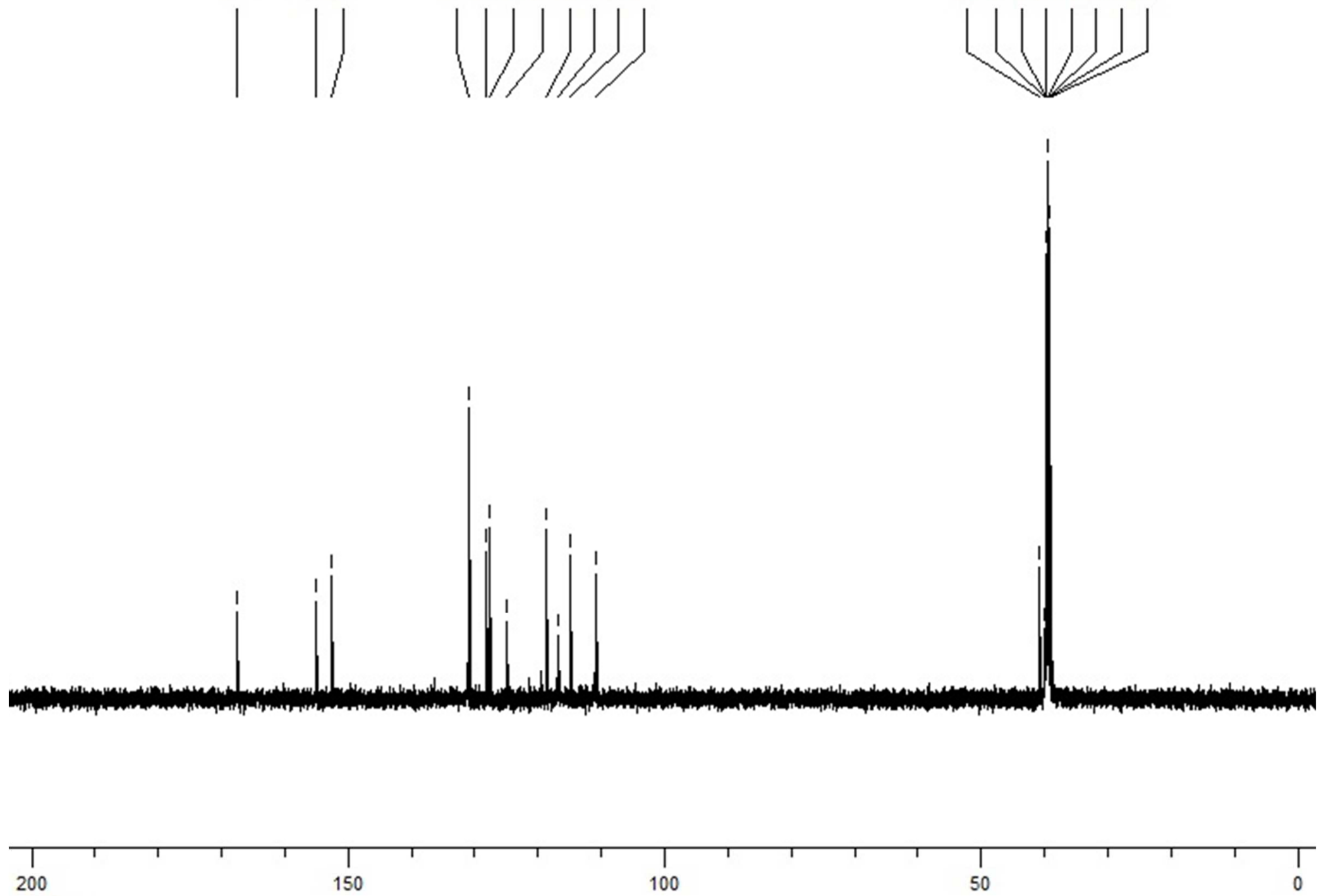
Figure S23. ${ }^{1} \mathrm{H}$ NMR and ${ }^{13} \mathrm{C}$ NMR spectra of N-Cinnamyl-4-methylaniline in $\mathrm{CDCl}_{3}$ (Table 1, Entry 21)
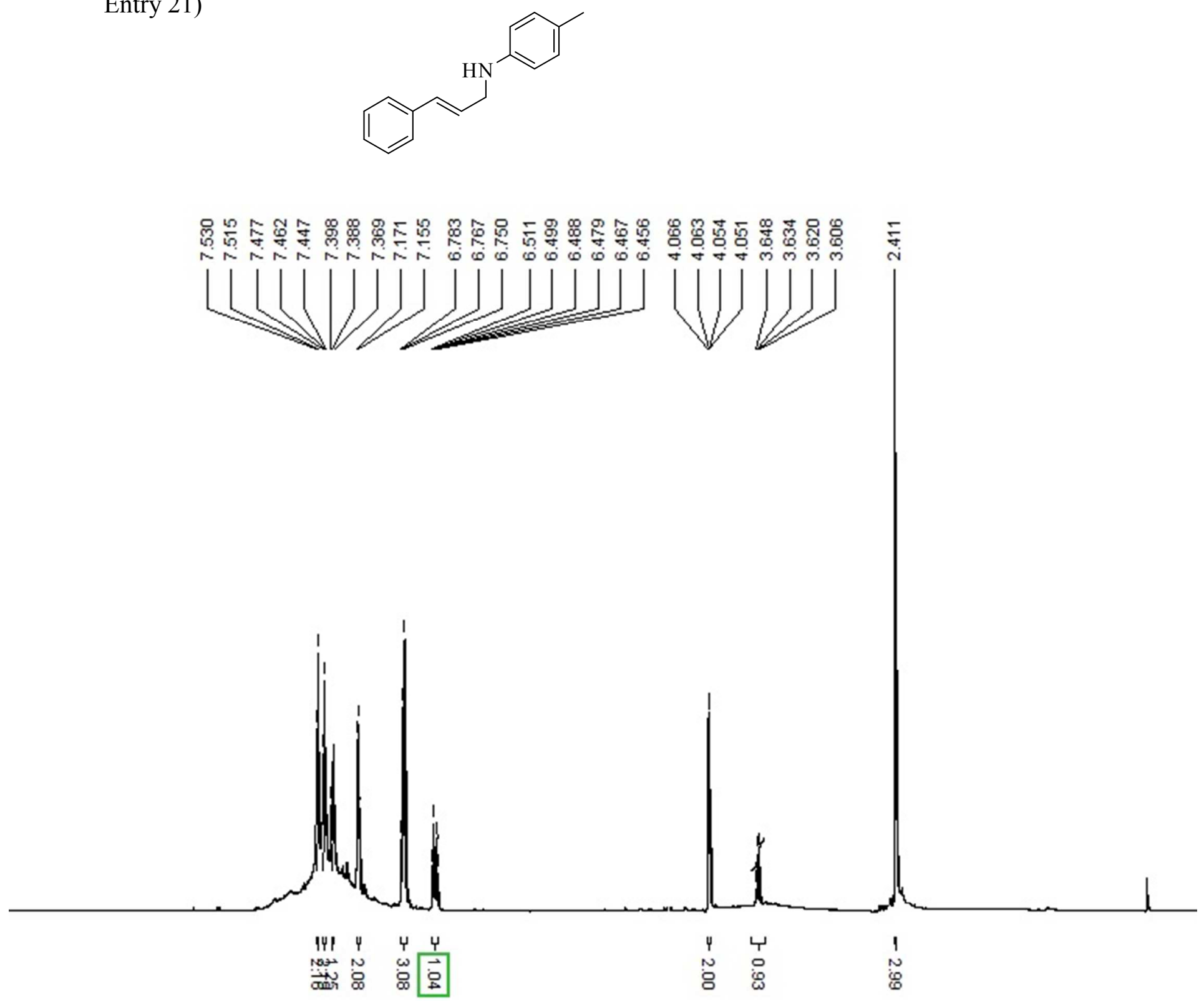

\begin{tabular}{c|c|c|c|c|c|}
\hline 1 & 1 & 1 & 1 & 1 \\
10.0 & 1 & 1 & 1
\end{tabular}



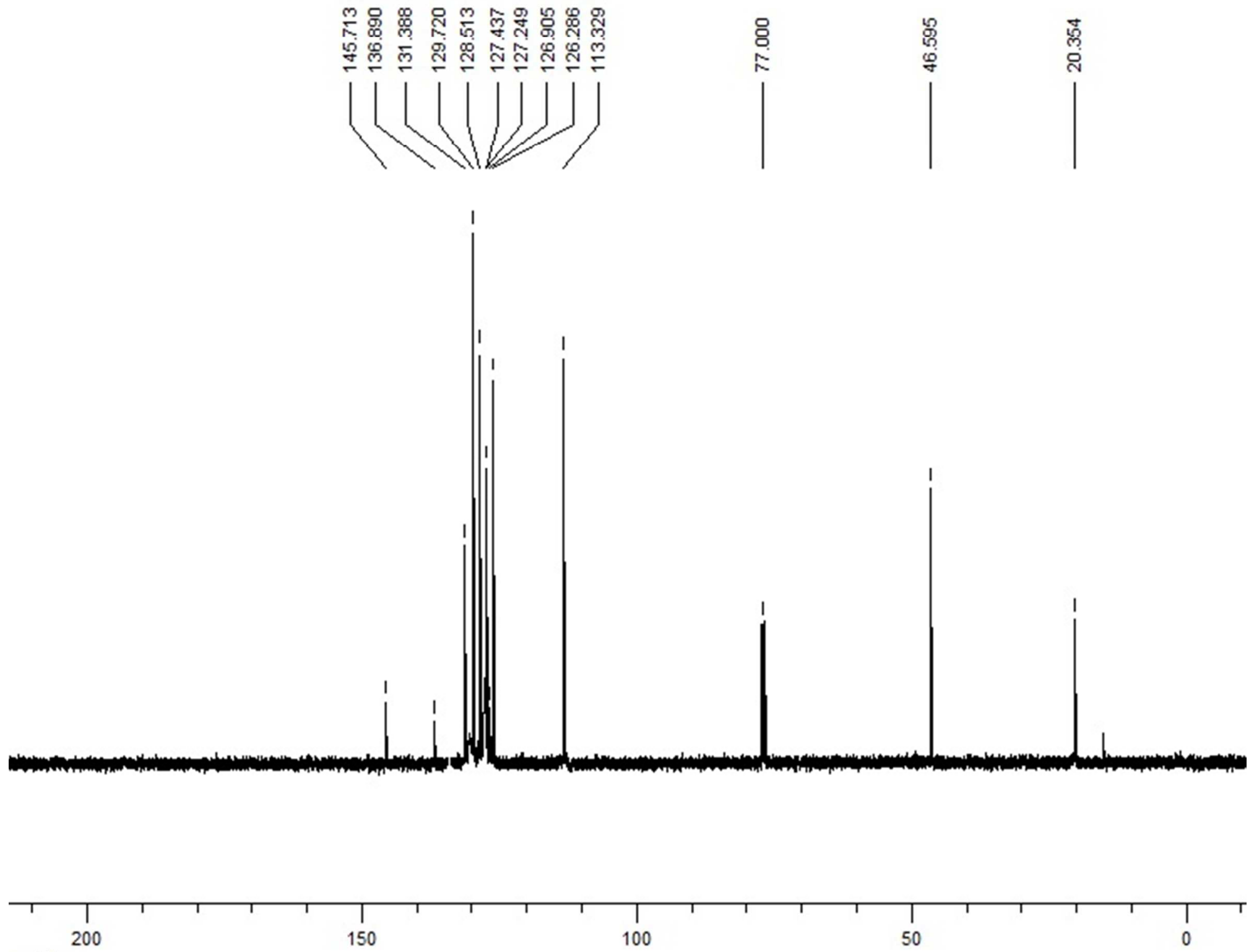
Figure S24. ${ }^{1} \mathrm{H}$ NMR and ${ }^{13} \mathrm{C}$ NMR spectra of 4-Methoxy-N-(pyridin-2-ylmethyl)aniline in $\mathrm{CDCl}_{3}$ (Table 1, Entry 22)<smiles>COc1ccc(NCc2ccccn2)cc1</smiles>

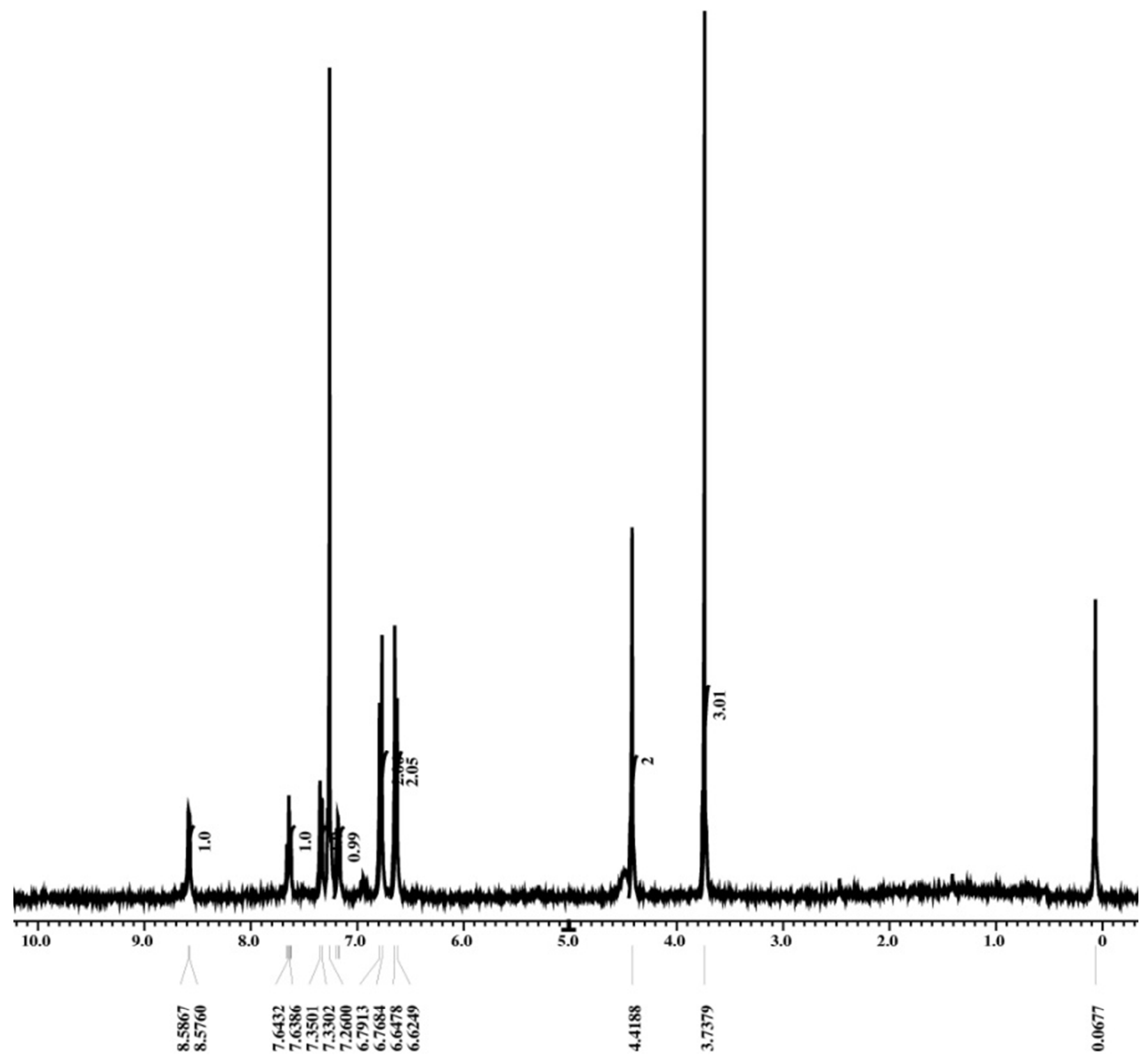




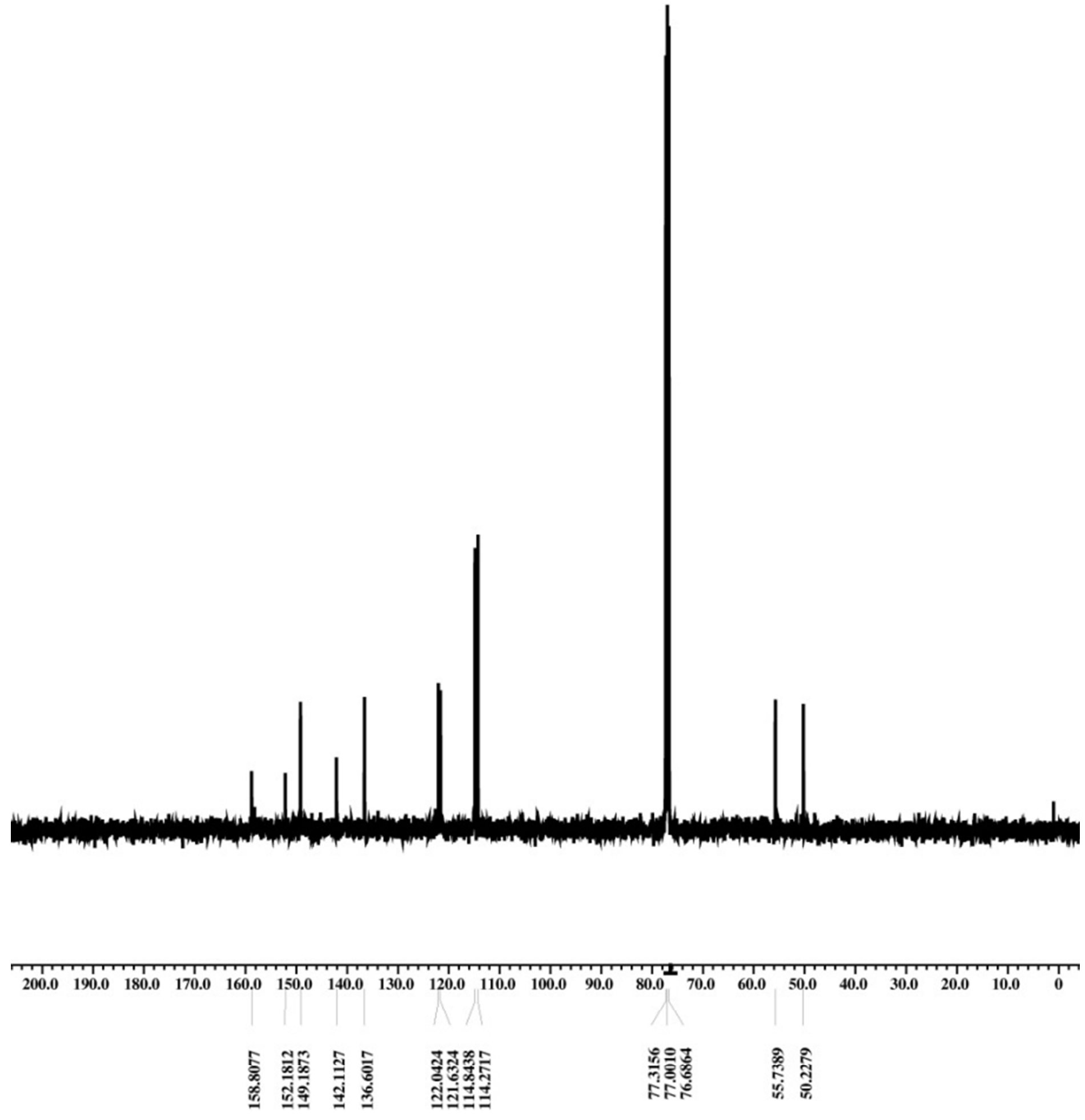


Figure S25. ${ }^{1} \mathrm{H}$ NMR and ${ }^{13} \mathrm{C}$ NMR spectra of N-(Anthracen-9-ylmethyl)-4-chloroaniline in $\mathrm{CDCl}_{3}$ (Table 1, Entry 24)<smiles>Clc1ccc(NCc2c3ccccc3cc3ccccc23)cc1</smiles>

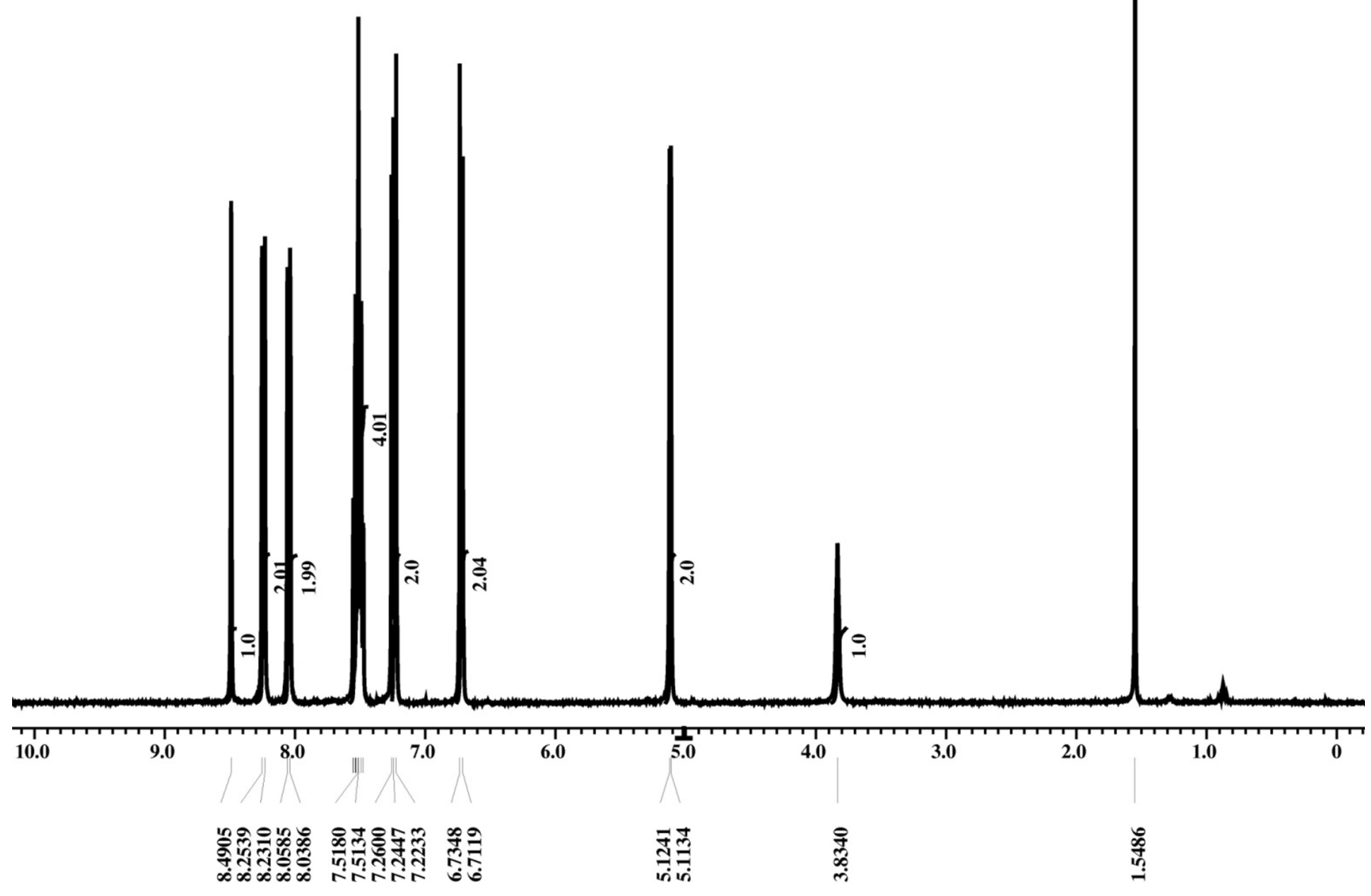




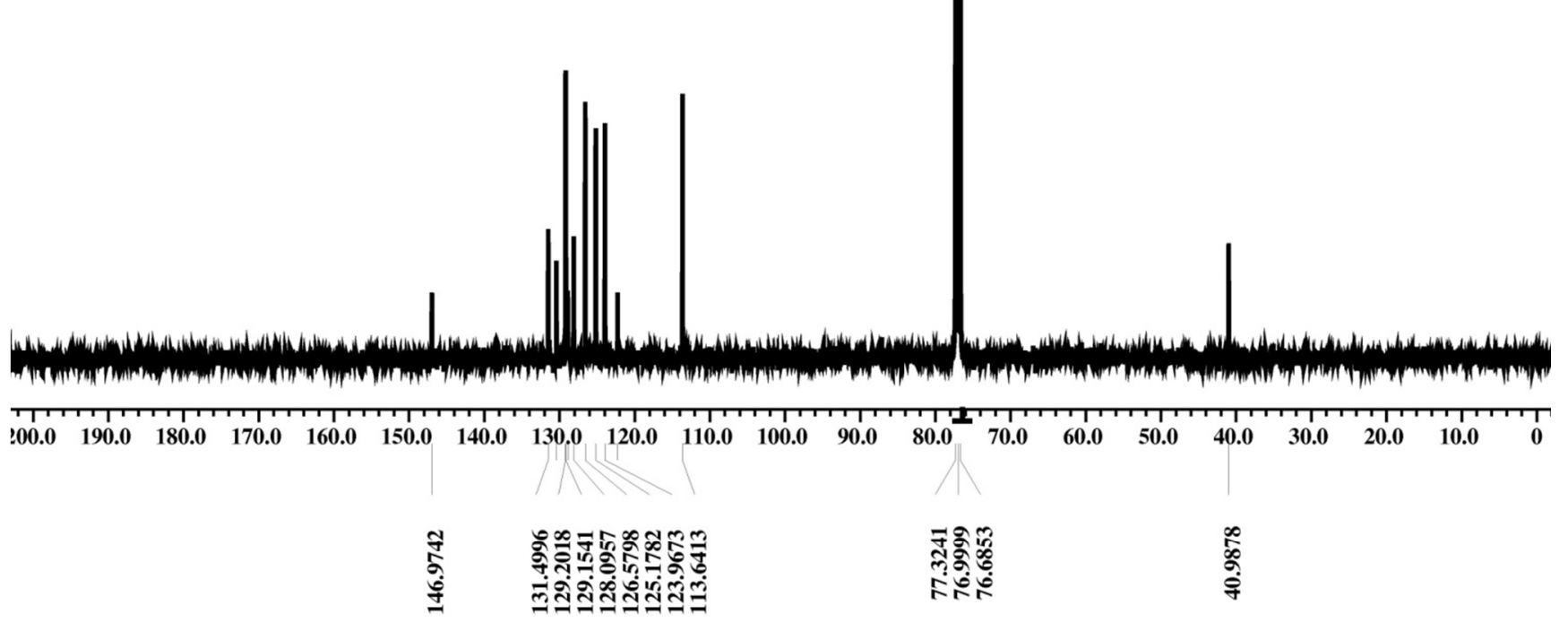

S71 
Figure S26. ${ }^{1} \mathrm{H}$ NMR and ${ }^{13} \mathrm{C}$ NMR spectra of 4-Methyl-N-(1-phenylethyl)aniline in $\mathrm{CDCl}_{3}$ (Table 2, Entry 1)<smiles>Cc1ccc(NC(C)c2ccccc2)cc1</smiles>

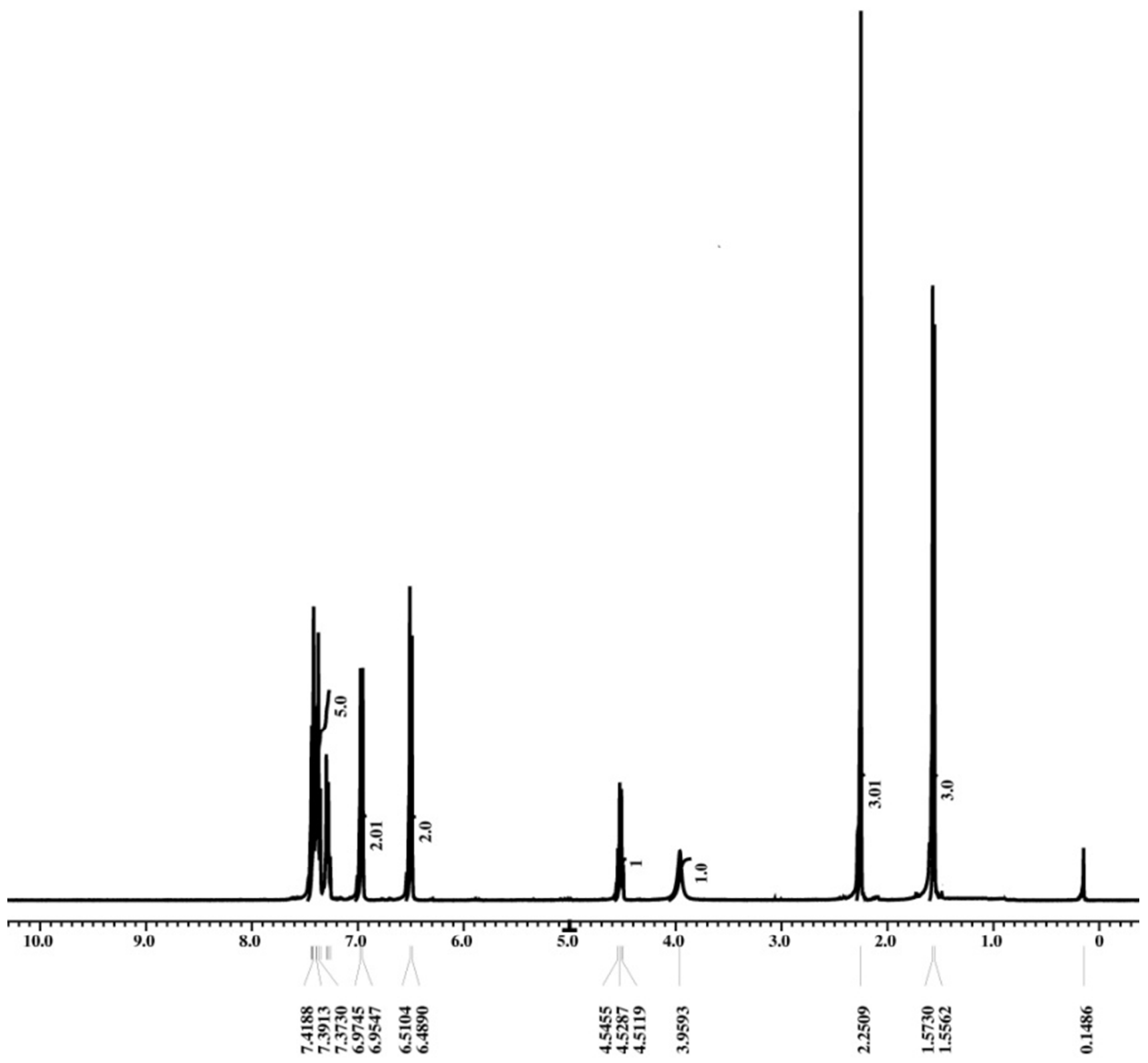




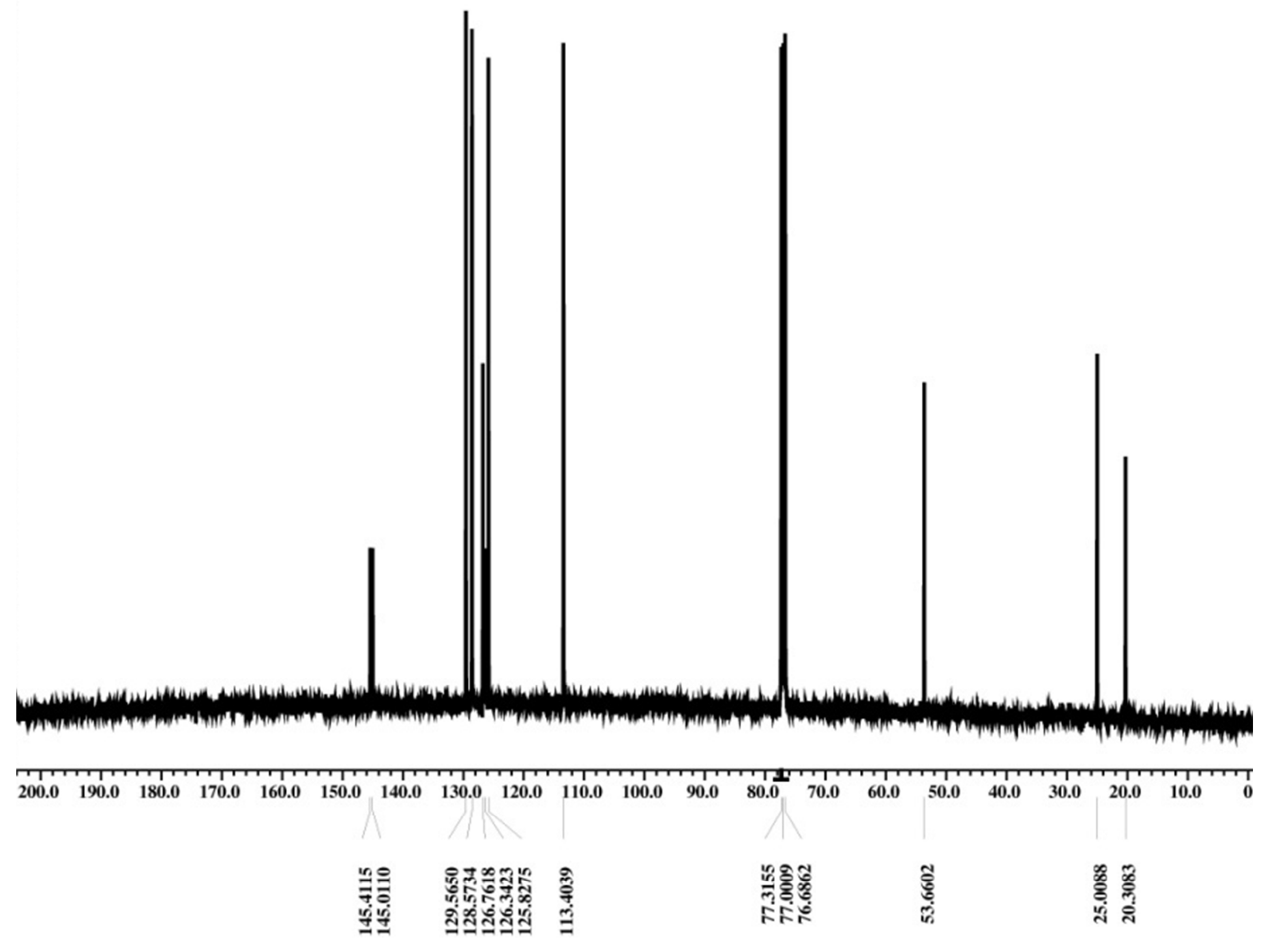


Figure S27. ${ }^{1} \mathrm{H}$ NMR and ${ }^{13} \mathrm{C}$ NMR spectra of 4-Methyl-N-[1-(4-methylphenyl)-ethyl]anilinein $\mathrm{CDCl}_{3}$ (Table 2, Entry 2)<smiles>Cc1ccc(NC(C)c2ccc(C)cc2)cc1</smiles>

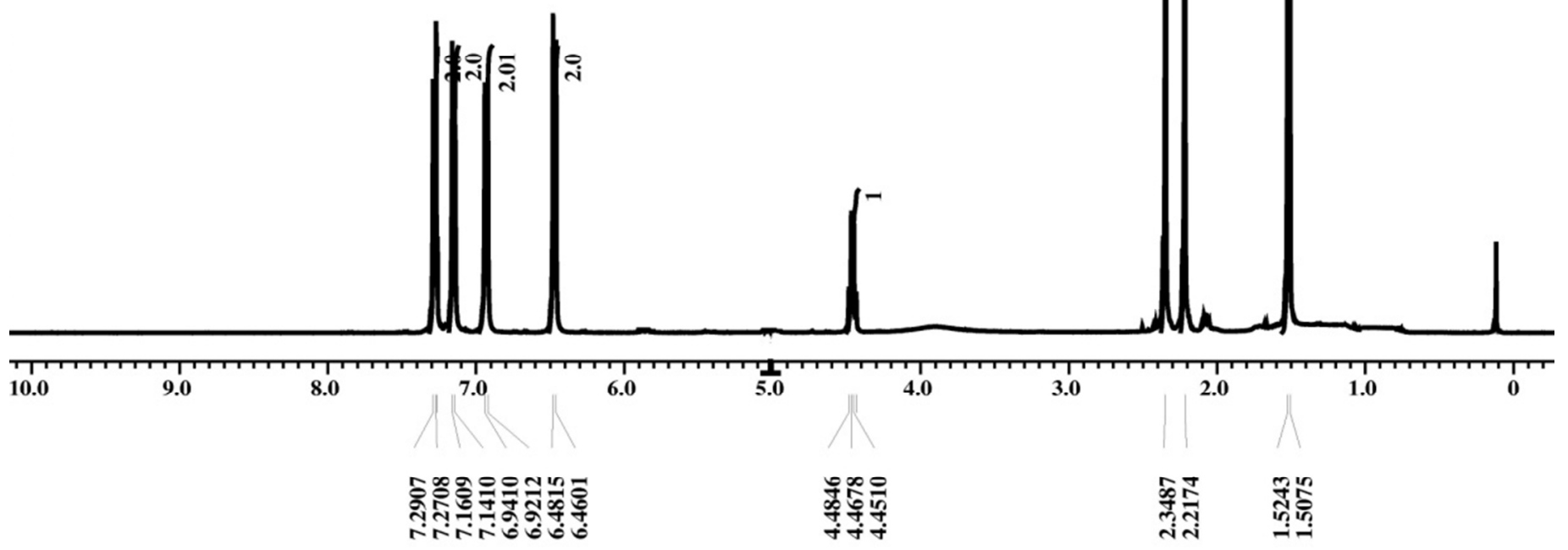




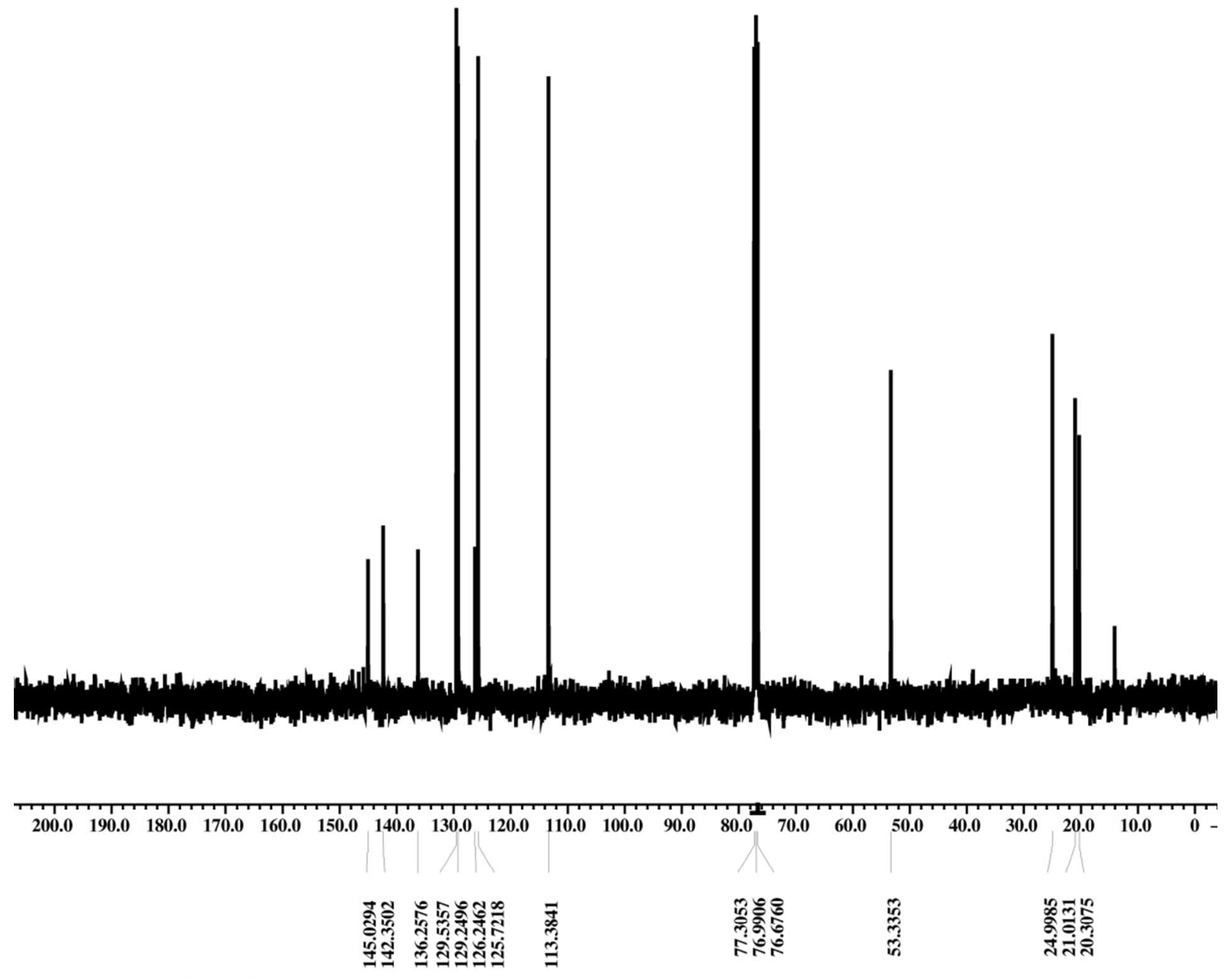


Figure S28. ${ }^{1} \mathrm{H}$ NMR and ${ }^{13} \mathrm{C}$ NMR spectra of N-\{1-[4-(Trifluoromethyl)phenyl]ethyl $\}-4-$ methylaniline in $\mathrm{CDCl}_{3}$ (Table 2, Entry 4)<smiles>Cc1ccc(NC(C)c2ccc(C(F)(F)F)cc2)cc1</smiles>
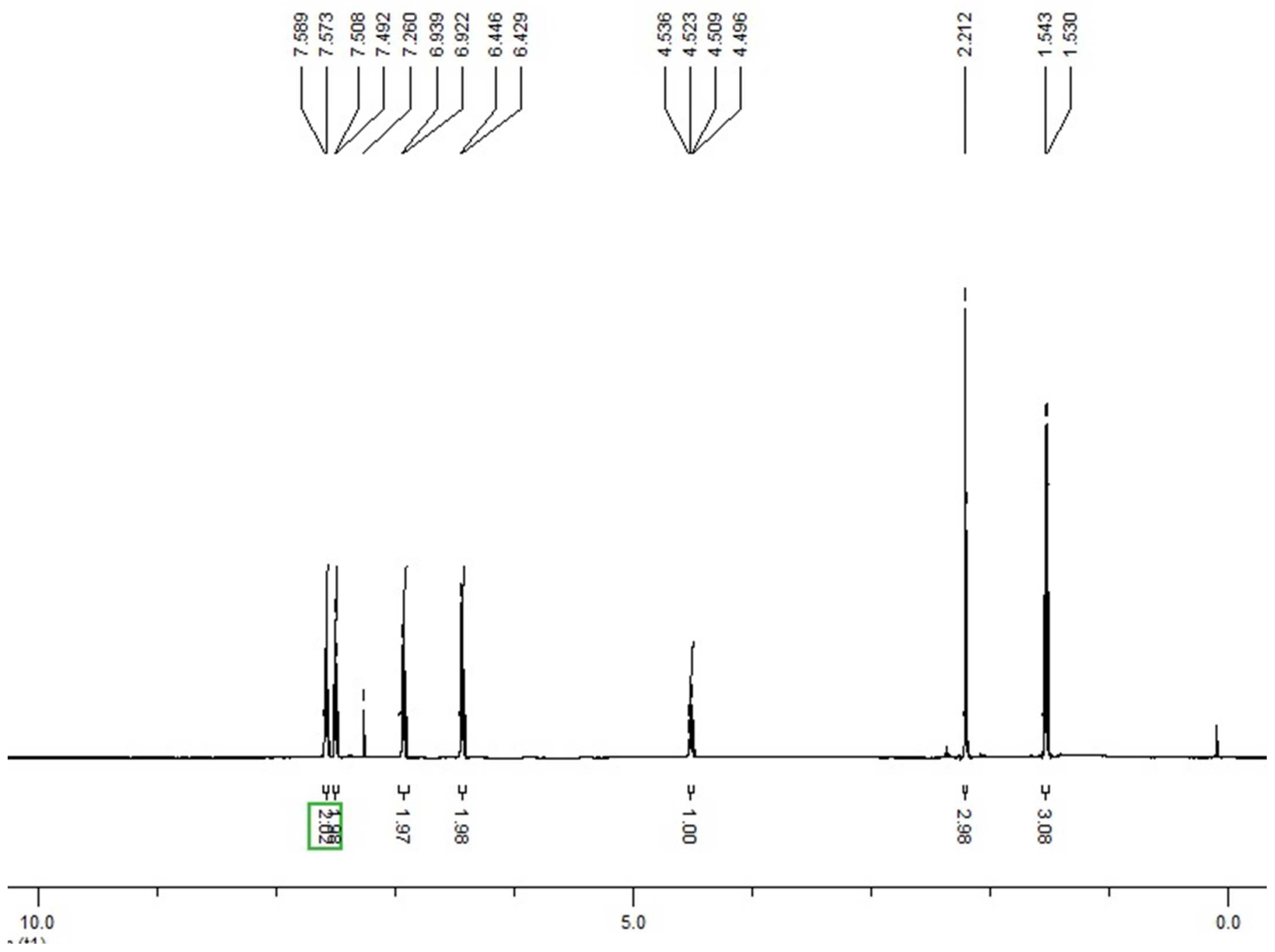


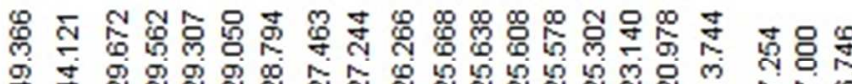

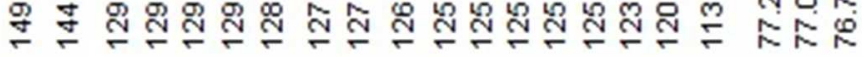
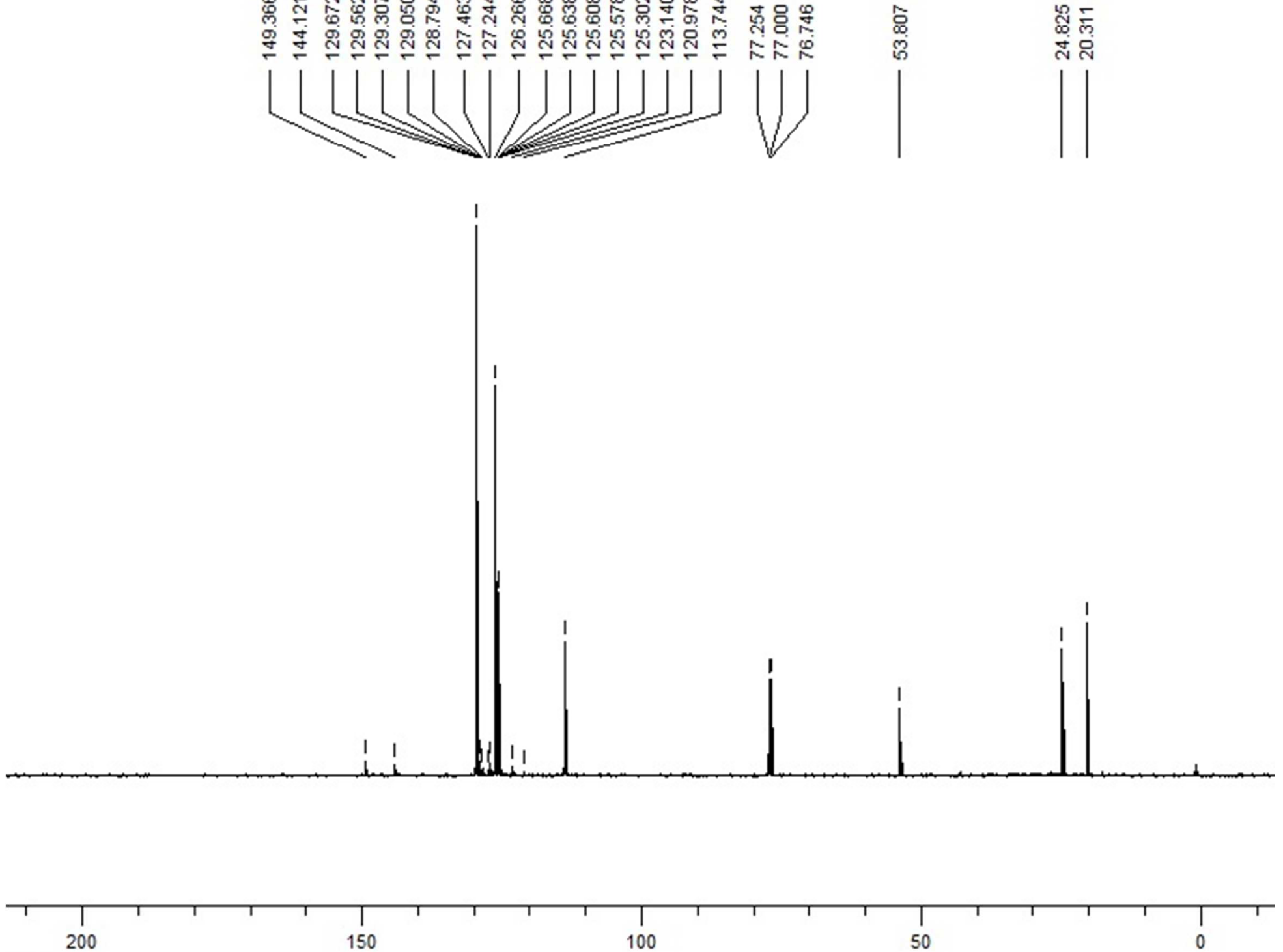
Figure S29. ${ }^{1} \mathrm{H}$ NMR and ${ }^{13} \mathrm{C}$ NMR spectra of 4-Methoxy-N-(1-phenylethyl)aniline in $\mathrm{CDCl}_{3}$ (Table 2, Entry 5)<smiles>COc1ccc(NC(C)c2ccccc2)cc1</smiles>

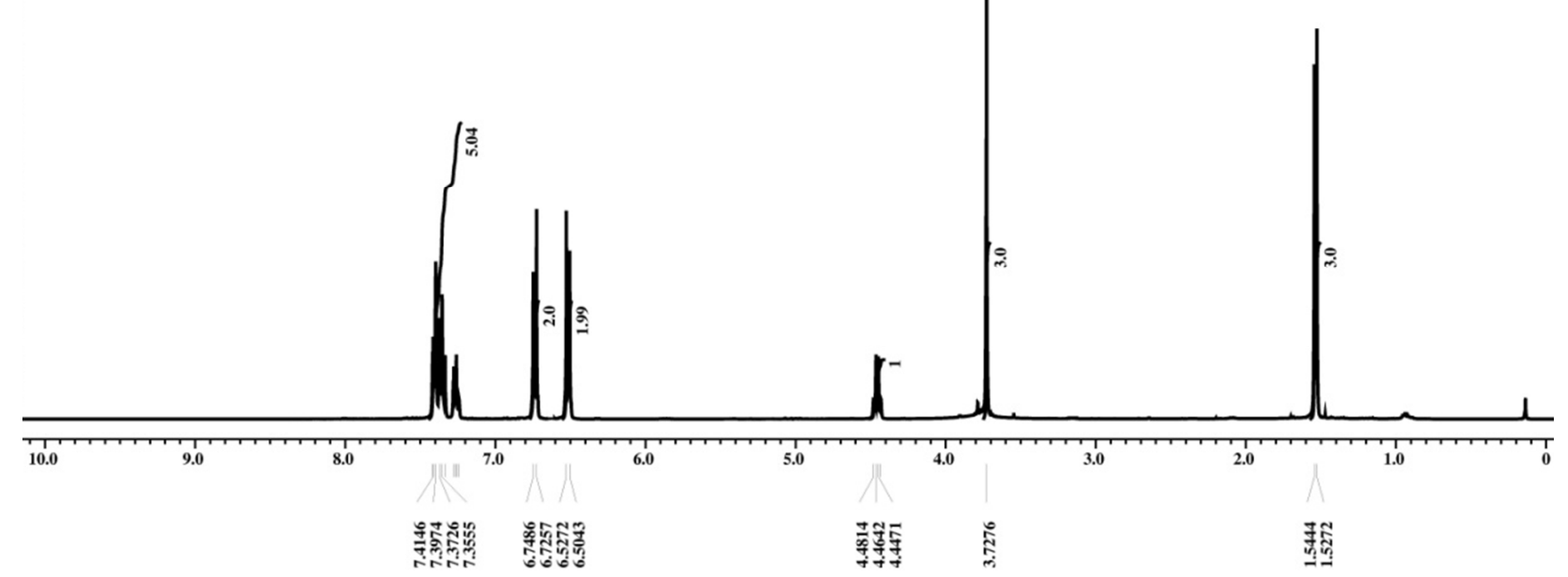




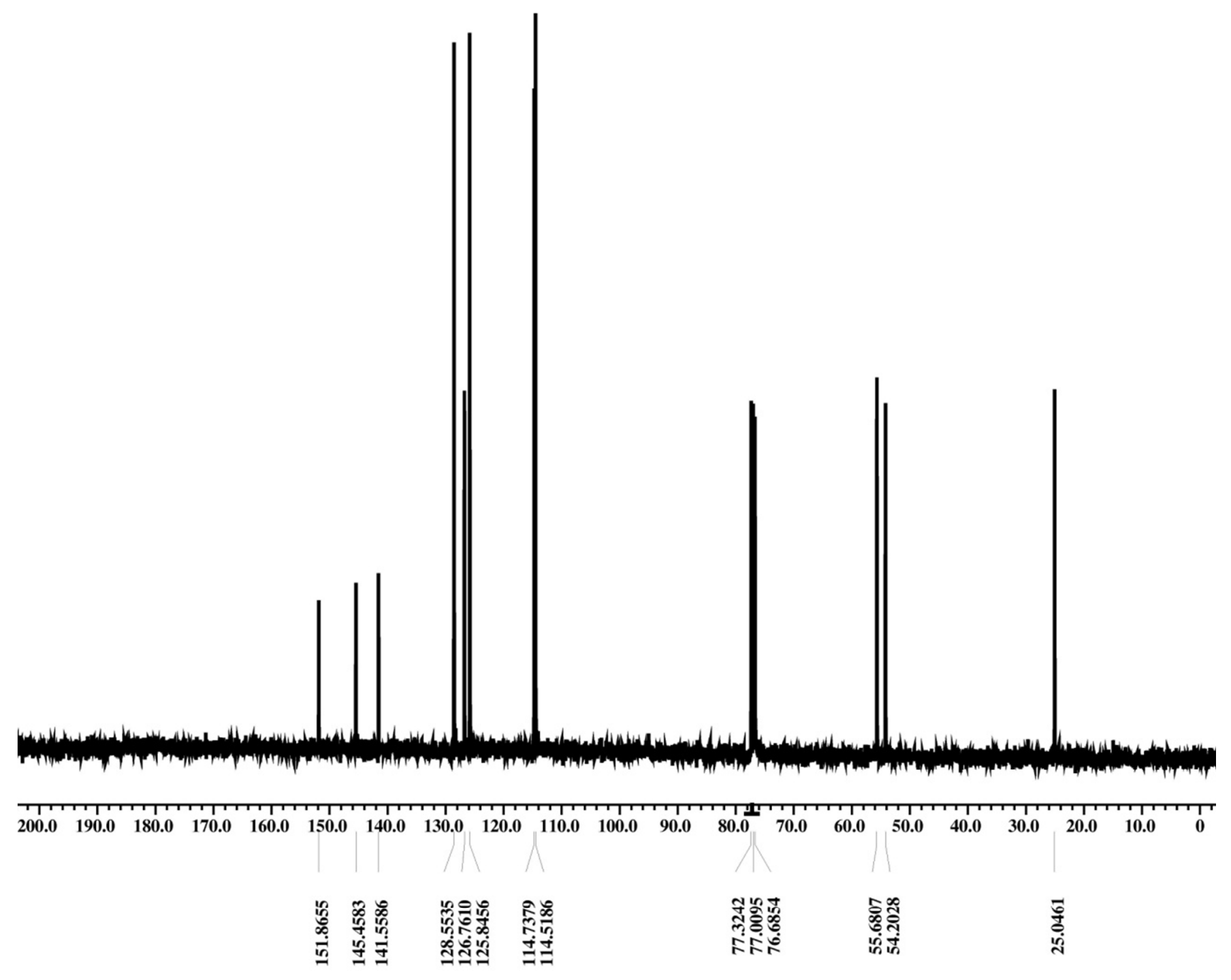


Figure S30. ${ }^{1} \mathrm{H}$ NMR and ${ }^{13} \mathrm{C}$ NMR spectra of N-[1-(4-(Trifluoromethyl)phenyl)ethyl]aniline in $\mathrm{CDCl}_{3}$ (Table 2, Entry 6)<smiles>CC(Nc1ccccc1)c1ccc(C(F)(F)F)cc1</smiles>

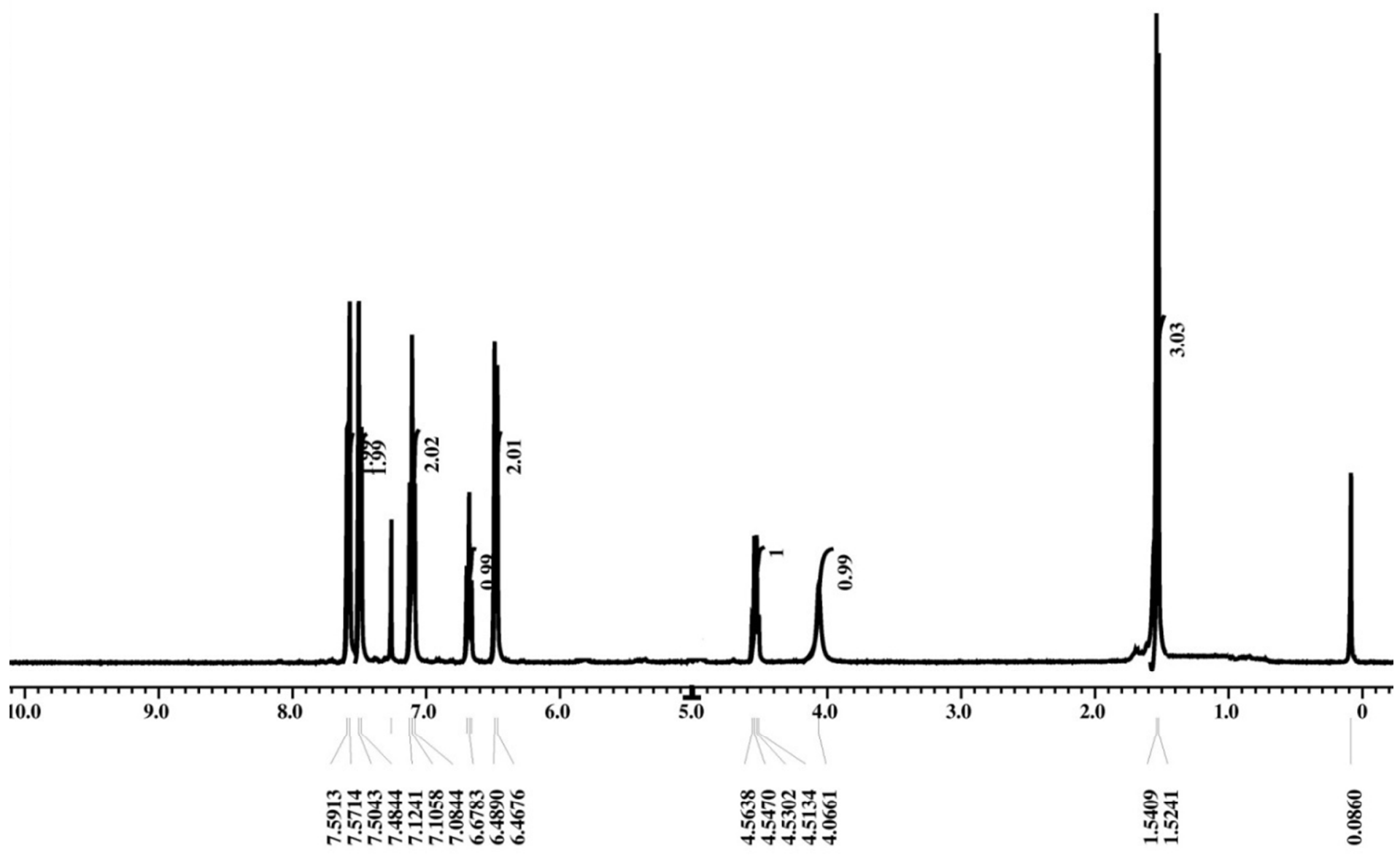



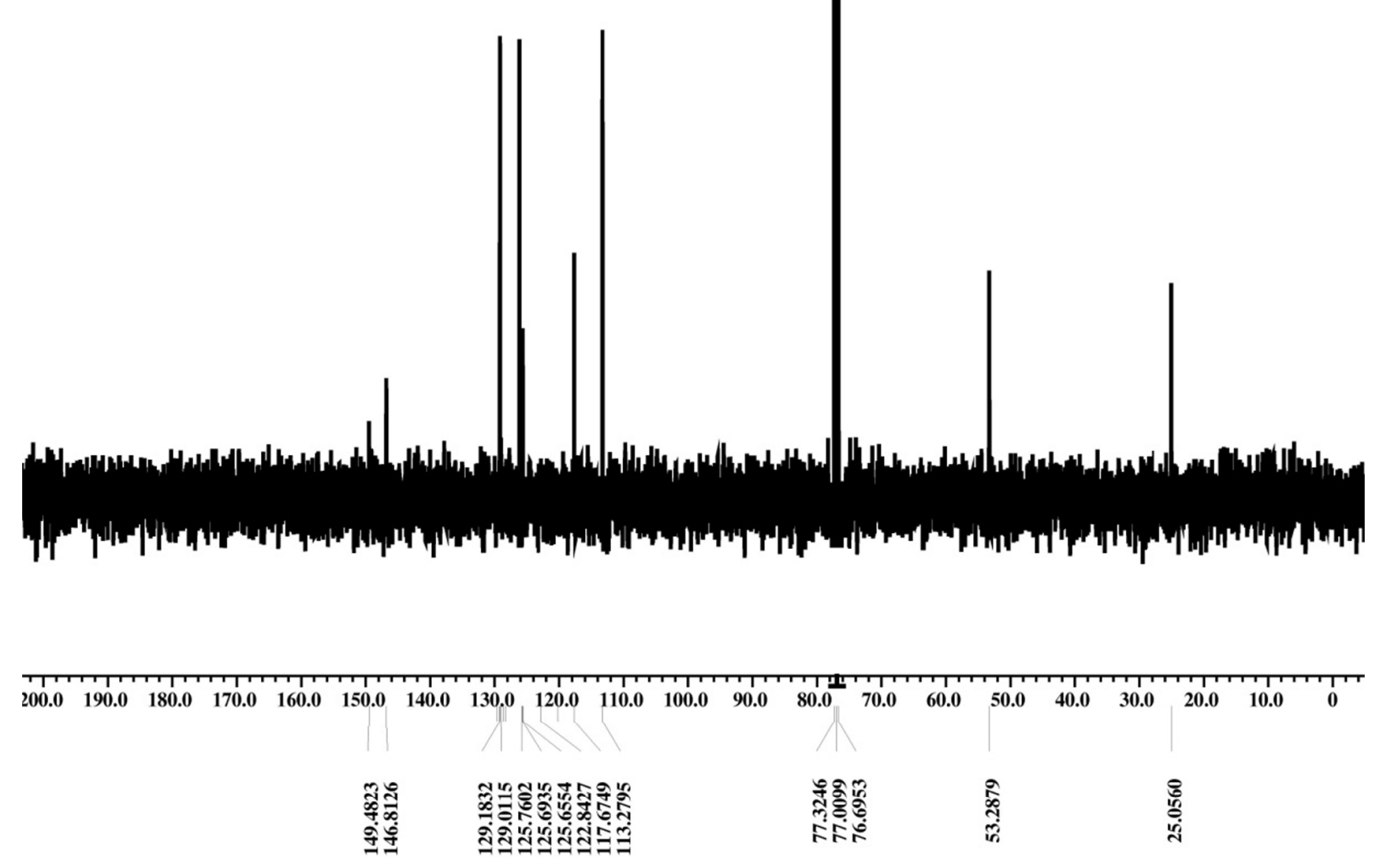

S81 
Figure S31. ${ }^{1} \mathrm{H}$ NMR and ${ }^{13} \mathrm{C}$ NMR spectra of N-[1-(2-methylphenyl)ethyl]aniline in $\mathrm{CDCl}_{3}$ (Table 2, Entry 7)

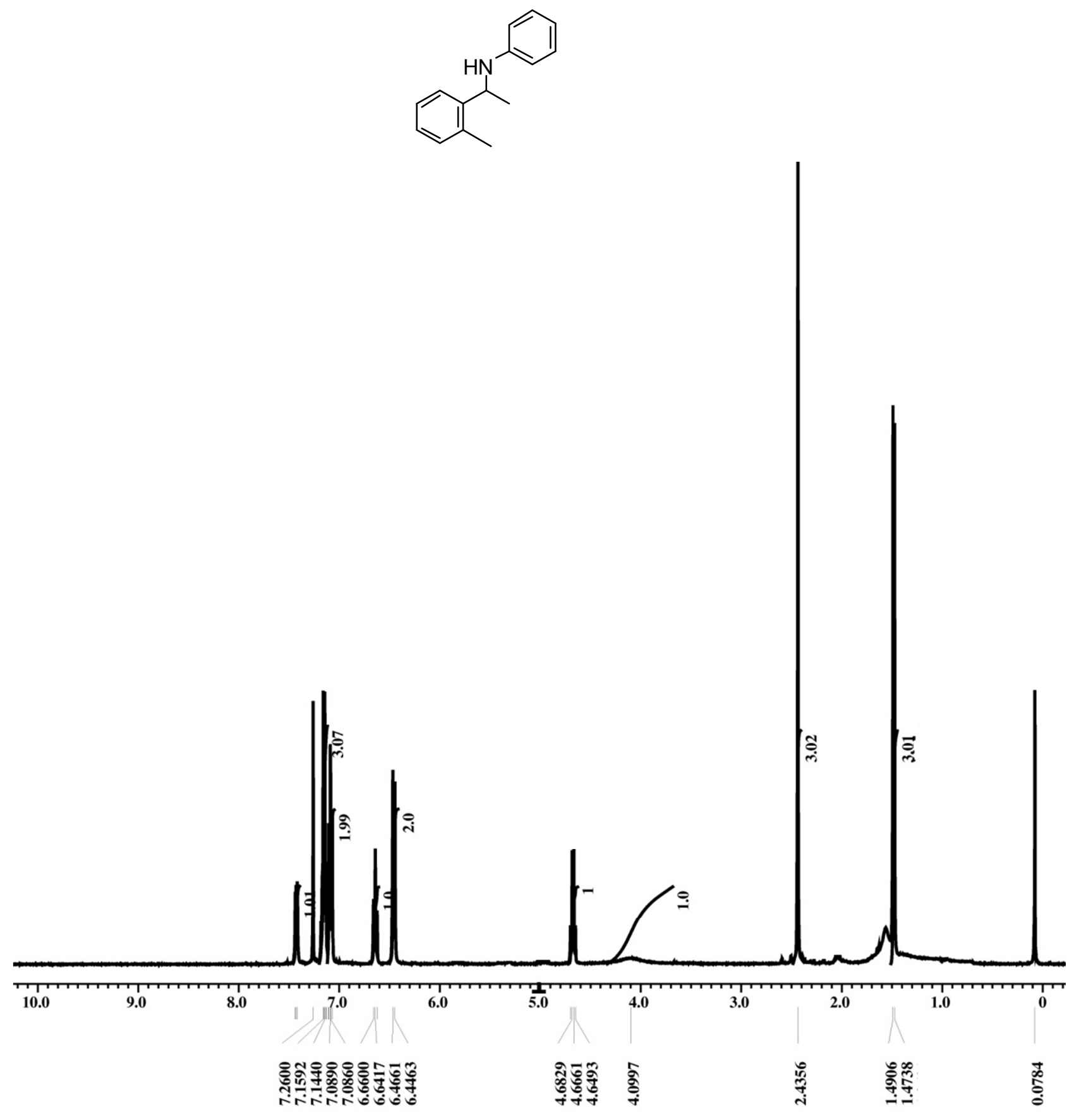




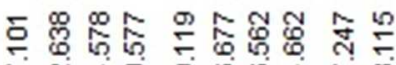

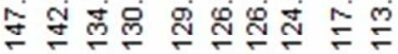

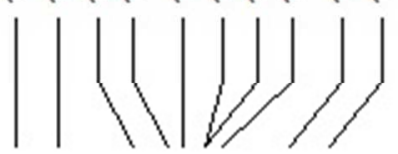

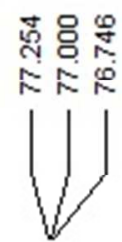
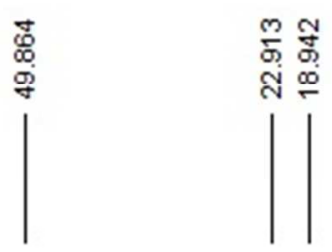
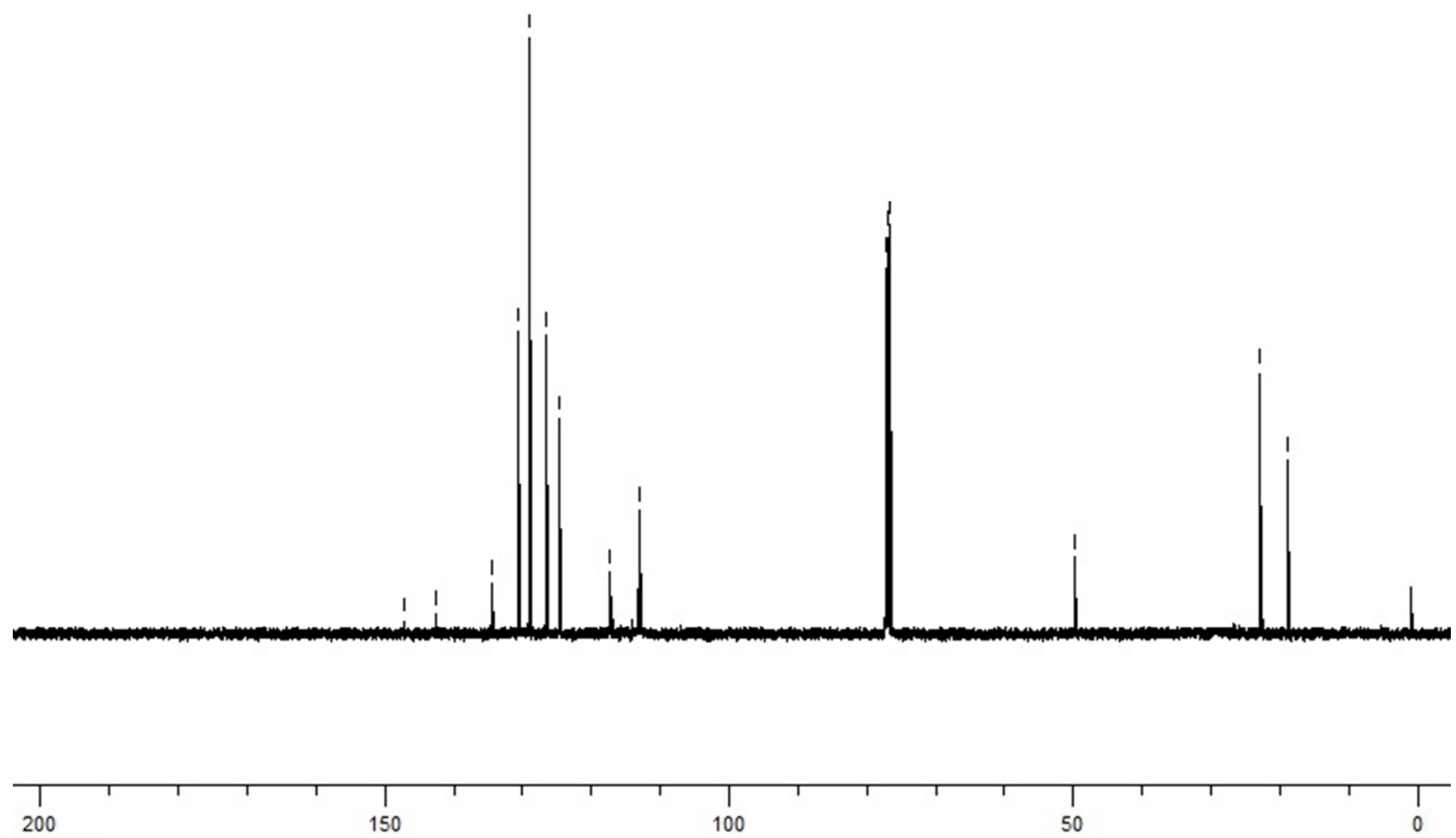
Figure S32. ${ }^{1} \mathrm{H}$ NMR and ${ }^{13} \mathrm{C}$ NMR spectra of N-[1-(1,2,3,4-tetrahydronaphthalenyl)aniline in $\mathrm{CDCl}_{3}$ (Table 2, Entry 8)<smiles>c1ccc(NC2CCCc3ccccc32)cc1</smiles>
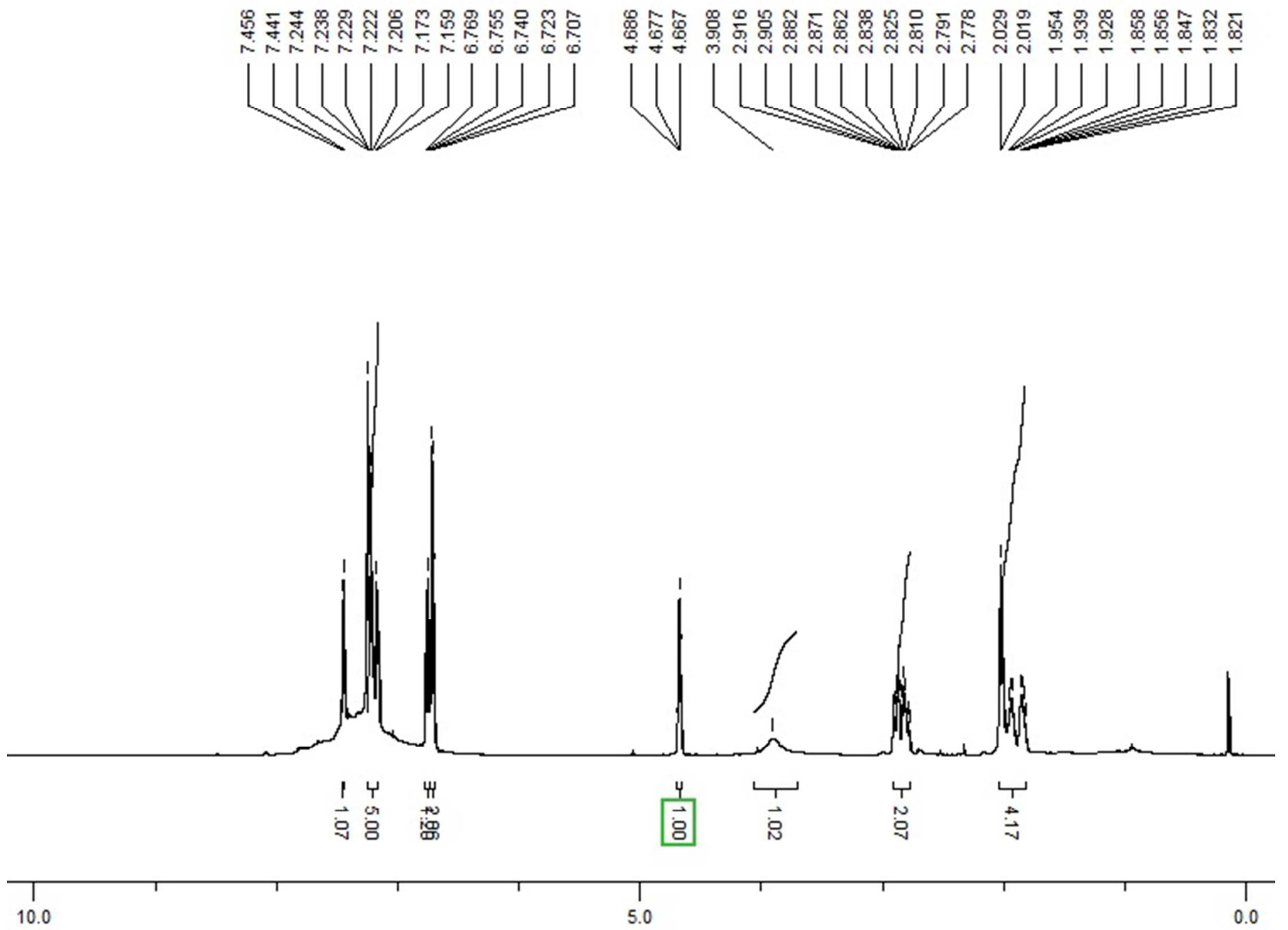

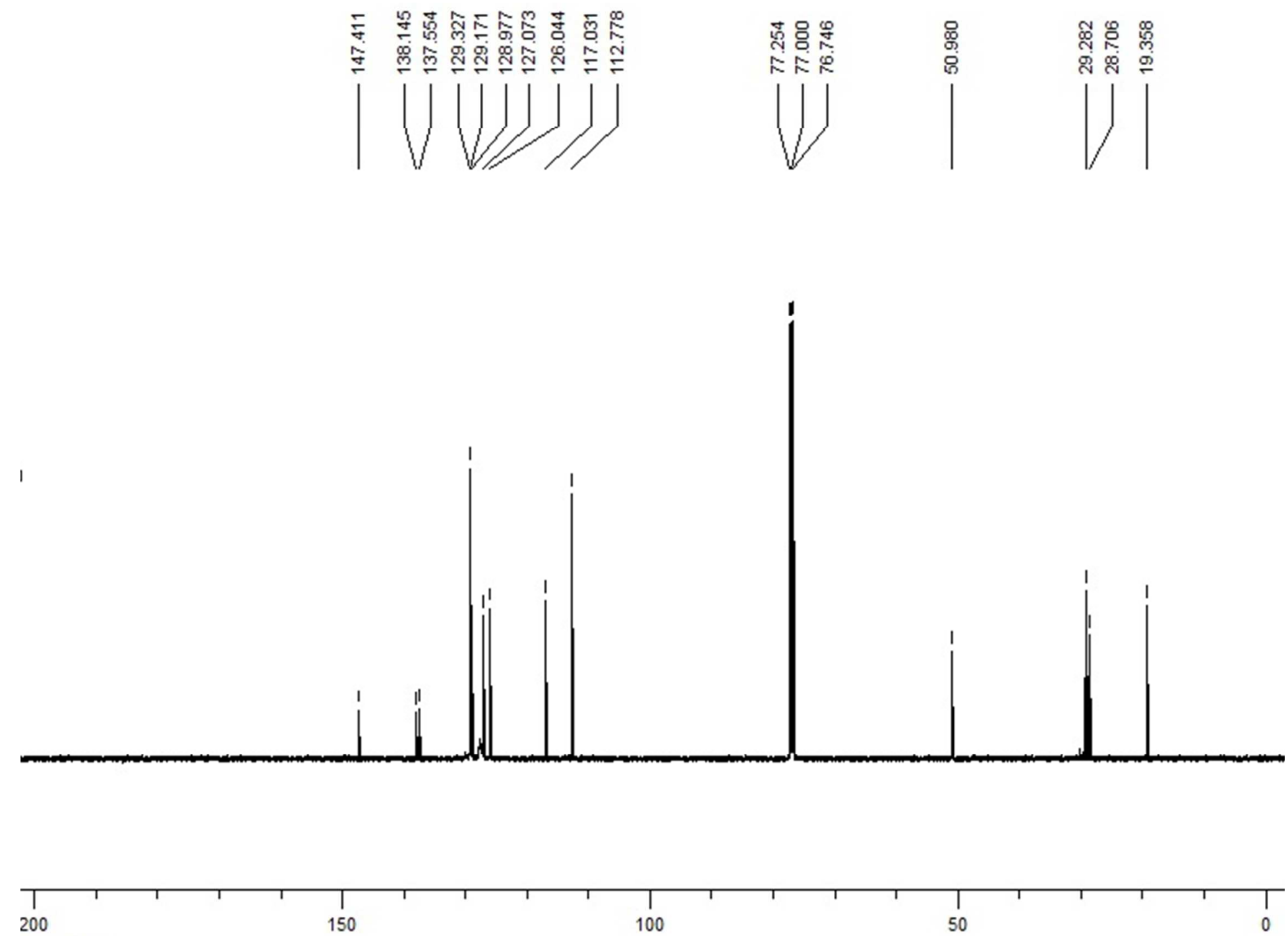
Figure S33. ${ }^{1} \mathrm{H} \quad \mathrm{NMR}$ and ${ }^{13} \mathrm{C}$ NMR spectra of N-benzyl-2,4,5-tris(benzyloxy)-6 (benzyloxymethyl)tetrahydro-2H-pyran-3-amine (7a) in DMSO-d $\mathrm{d}_{6}$ :

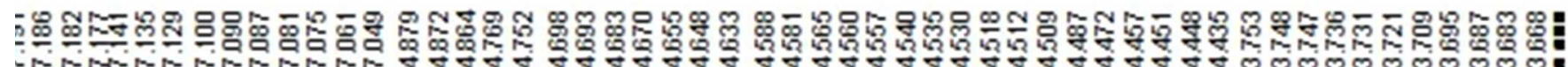
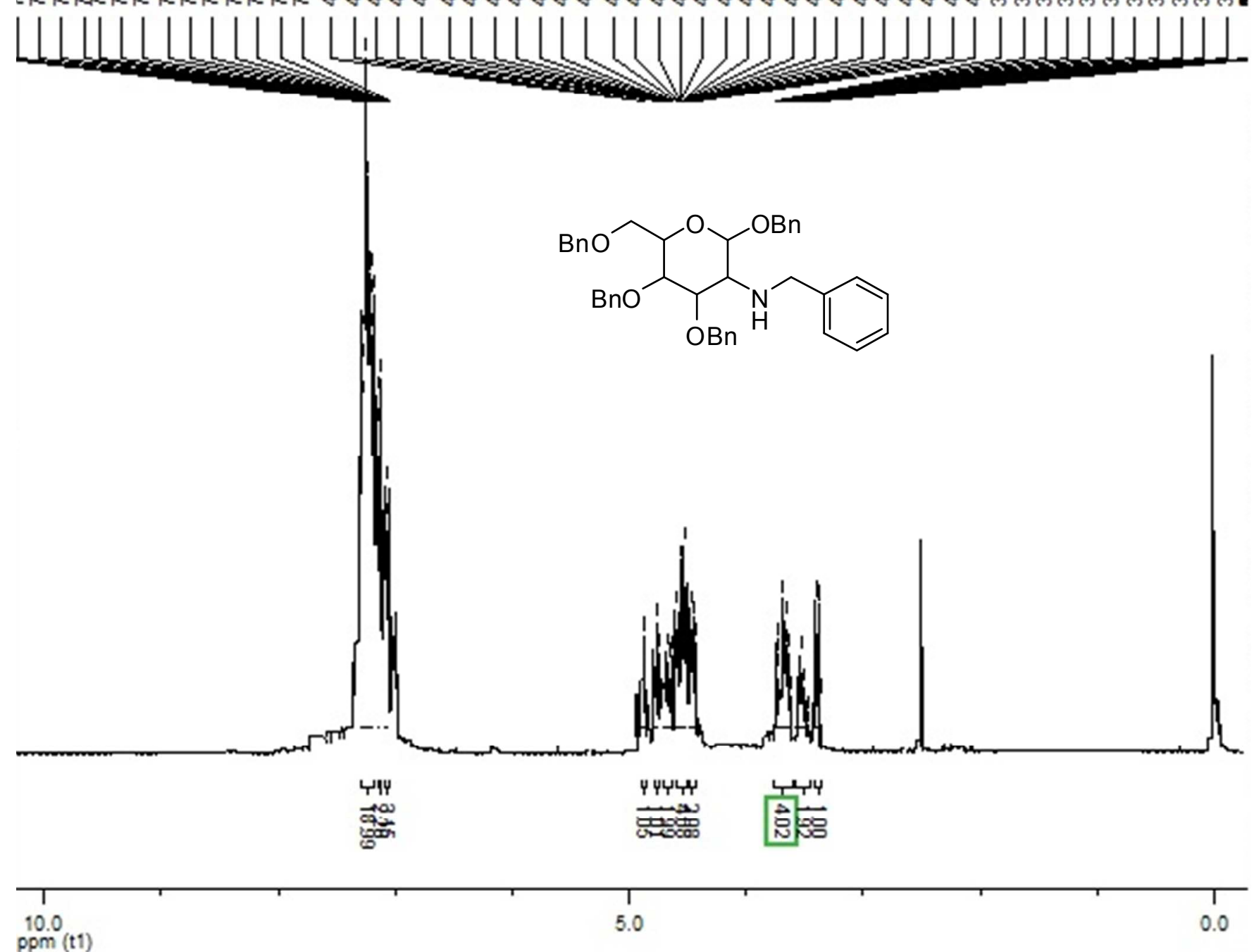


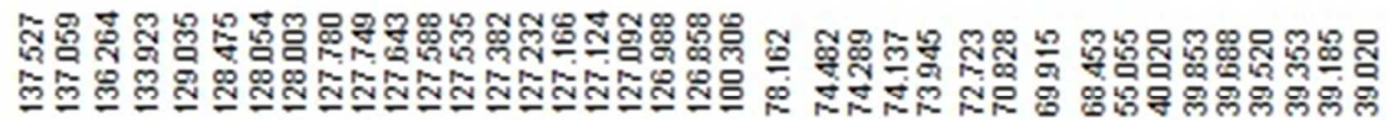
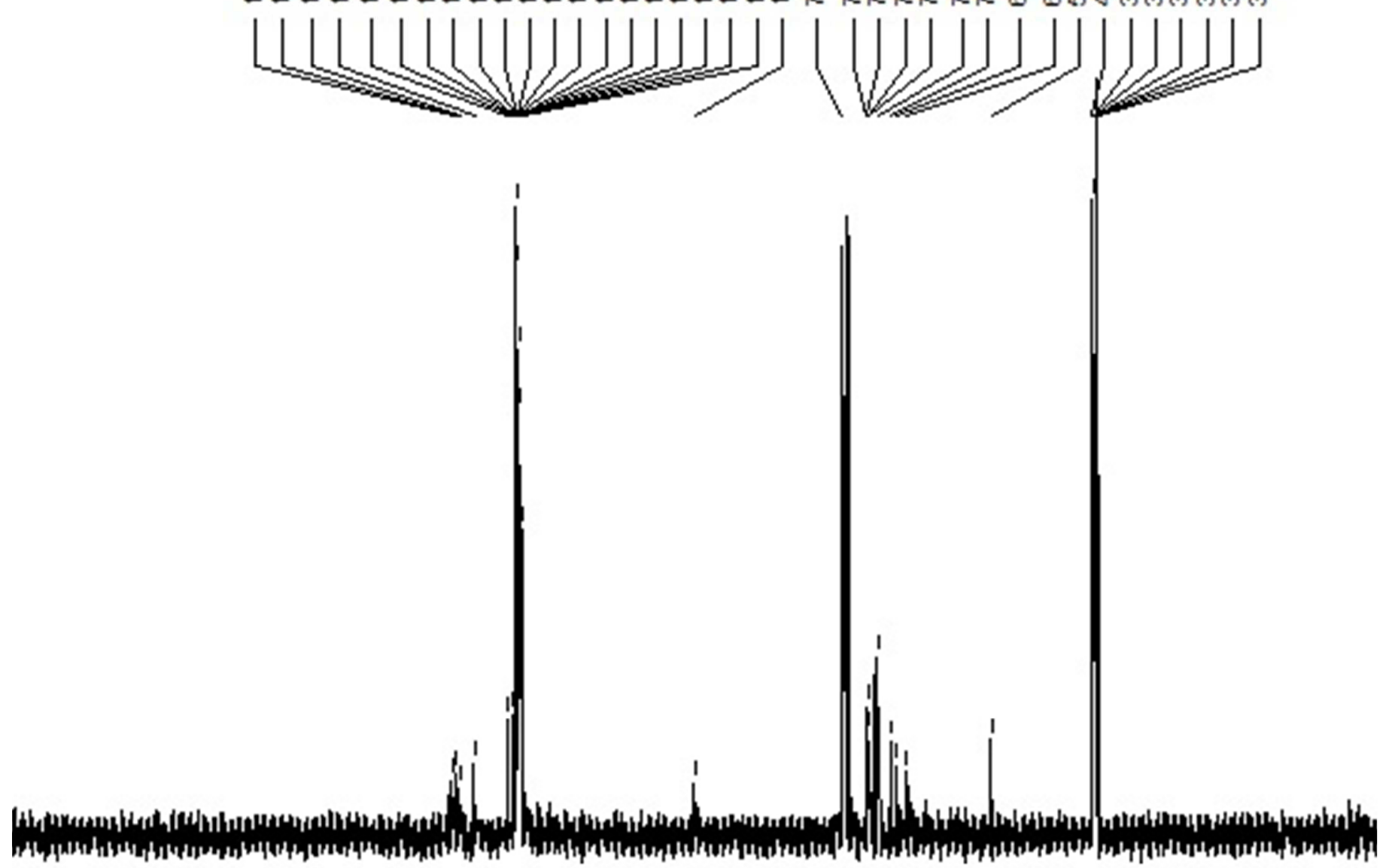

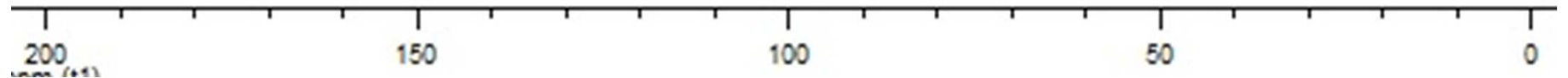


Figure S34. ${ }^{1} \mathrm{H}$ NMR and ${ }^{13} \mathrm{C}$ NMR spectra of 2,4,5-tris(benzyloxy)-6-(benzyloxymethyl)-N-(4methylbenzyl)tetrahydro-2H-pyran-3-amine (7b) in $\mathrm{CDCl}_{3}$ :
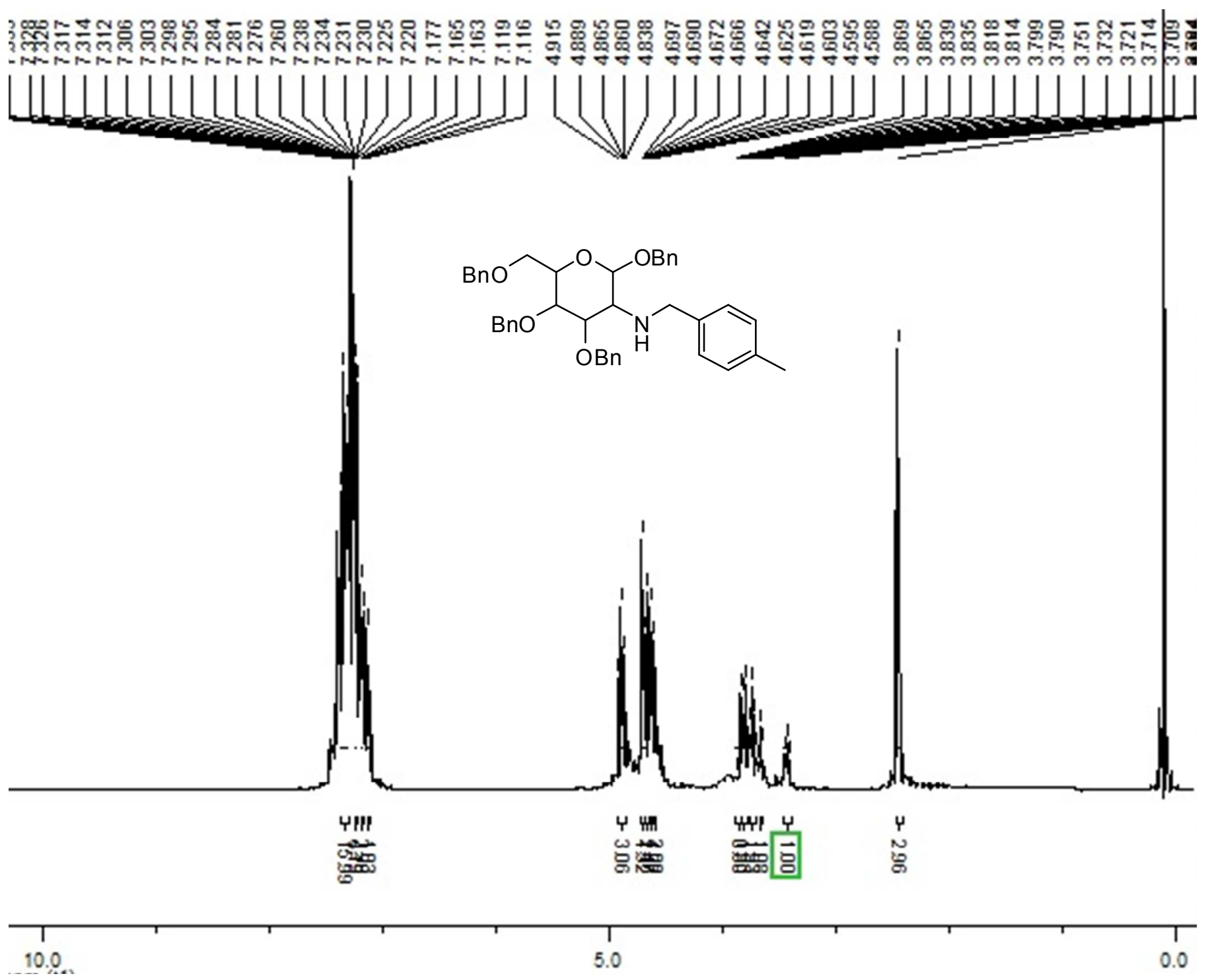

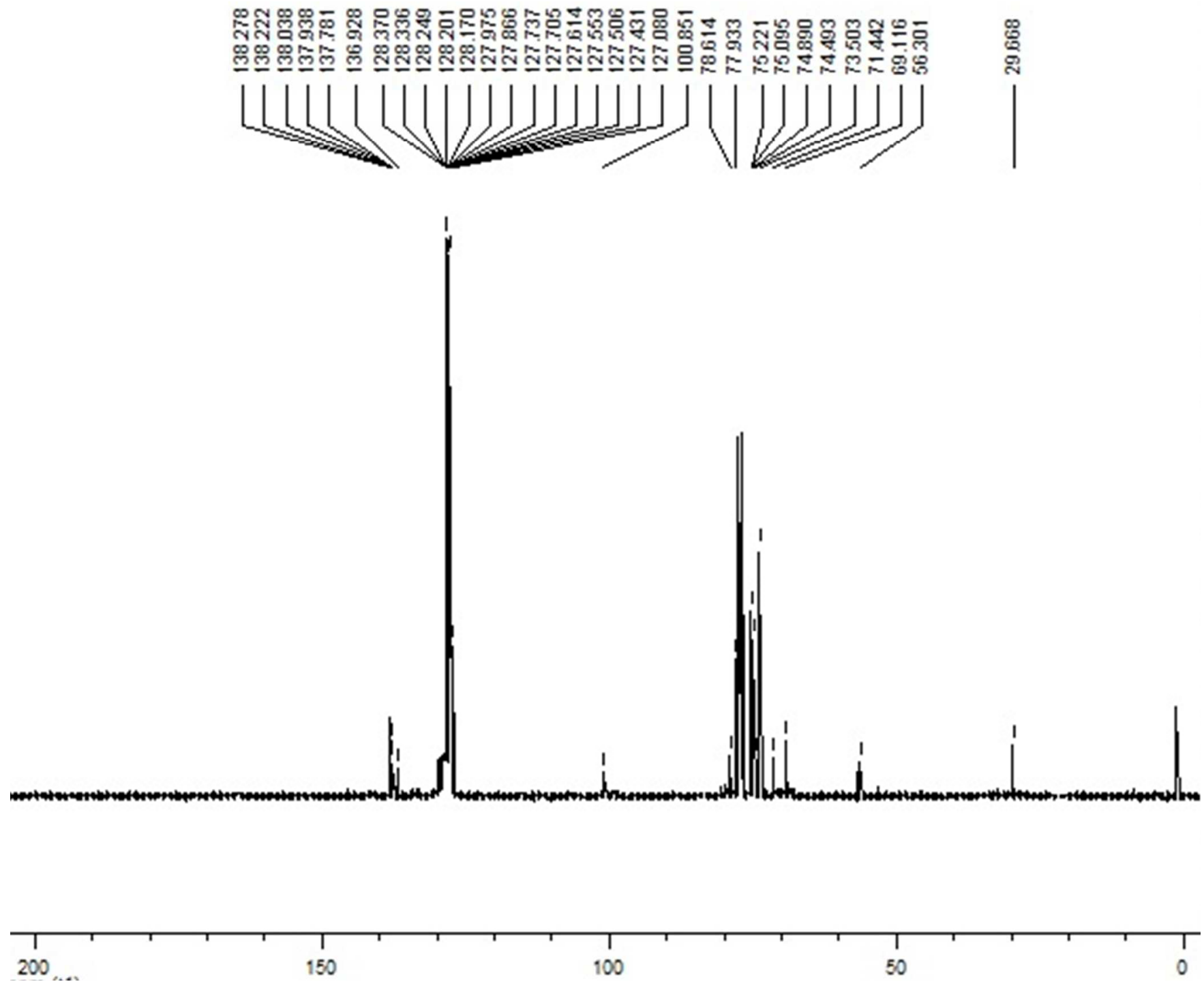
Figure S35. ${ }^{1} \mathrm{H}$ NMR and ${ }^{13} \mathrm{C}$ NMR spectra of 2,4,5-tris(benzyloxy)-6-(benzyloxymethyl)-N-(4chlorobenzyl)tetrahydro-2H-pyran-3-amine (7c) in $\mathrm{CDCl}_{3}$ :
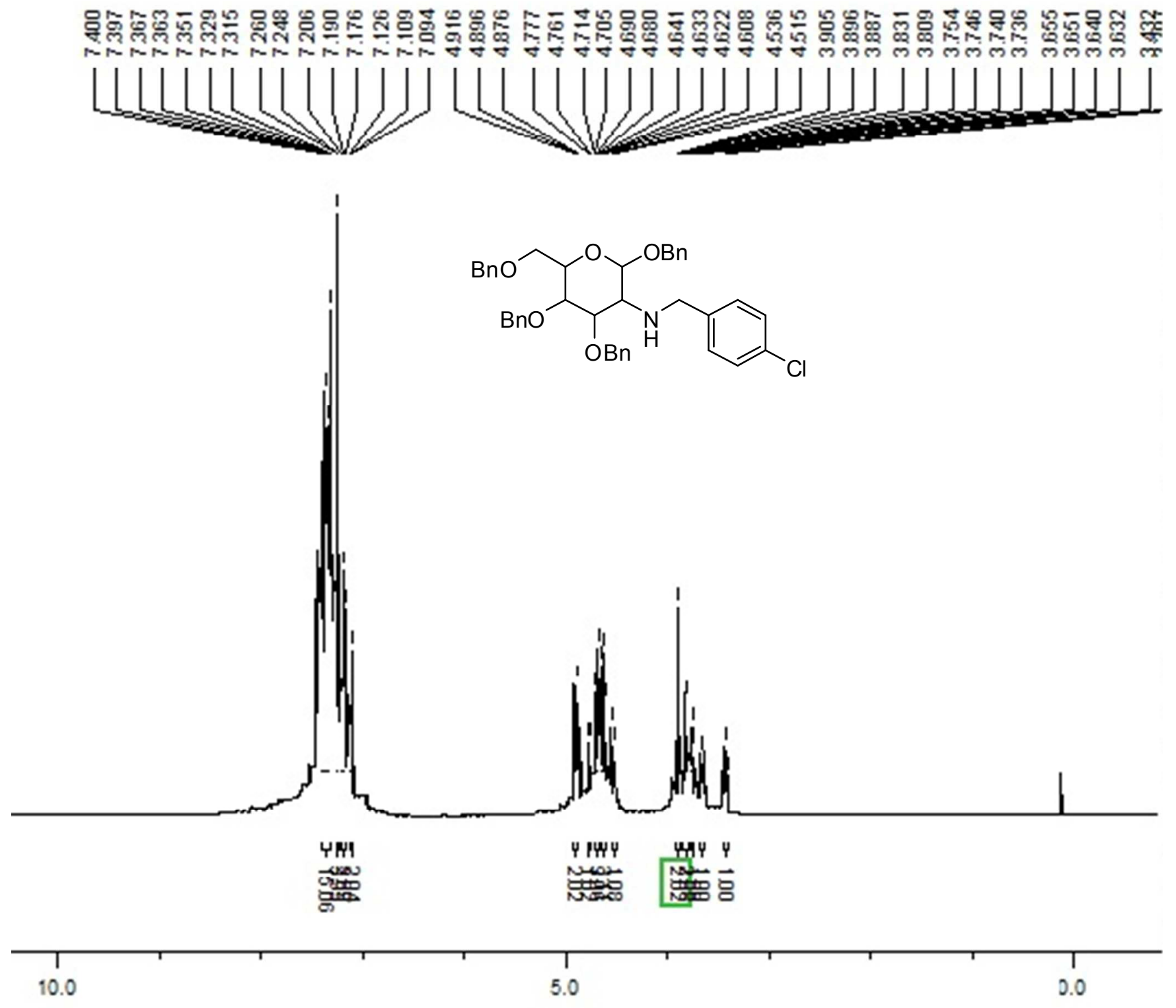

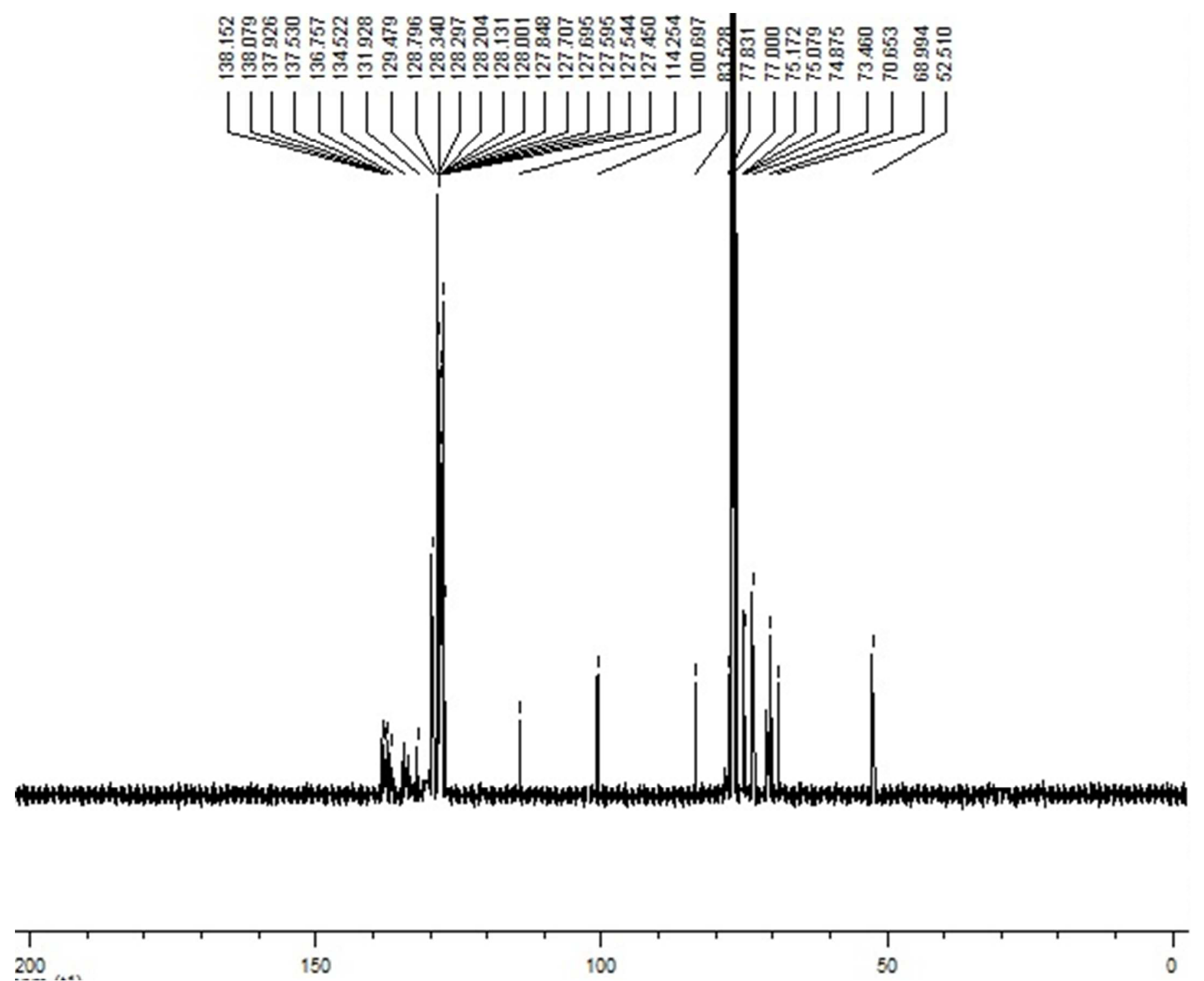
Figure S36. ${ }^{1} \mathrm{H}$ NMR and ${ }^{13} \mathrm{C}$ NMR spectra of 2,4,5-tris(benzyloxy)-6-(benzyloxymethyl)-N((5-methylthiophen-2-yl)methyl)tetrahydro-2H-pyran-3-amine (7d) in $\mathrm{CDCl}_{3}$ :

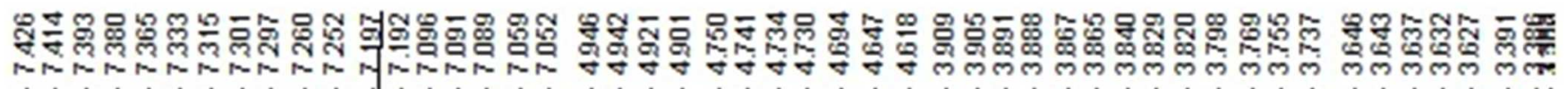
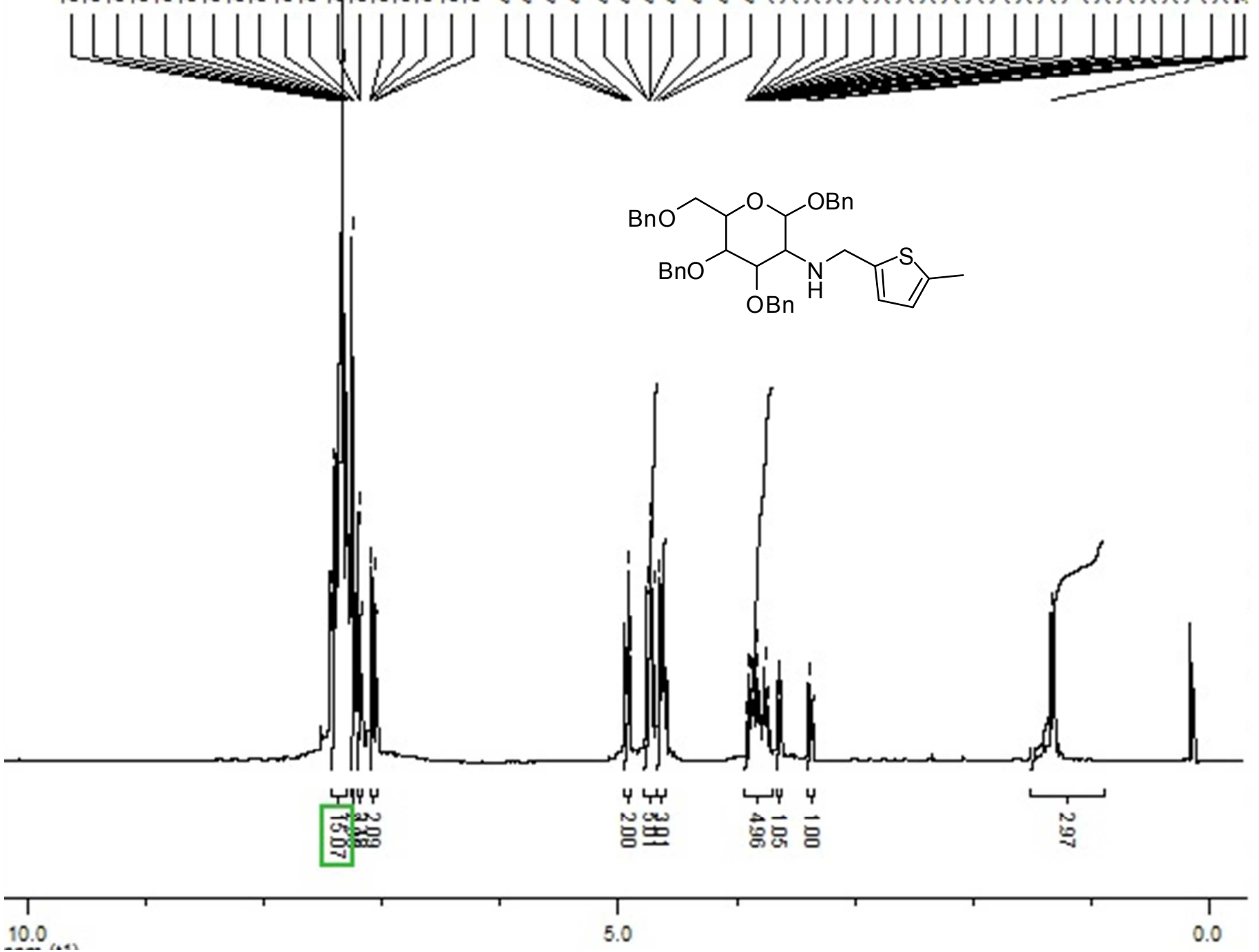


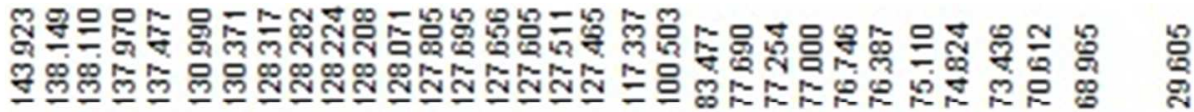
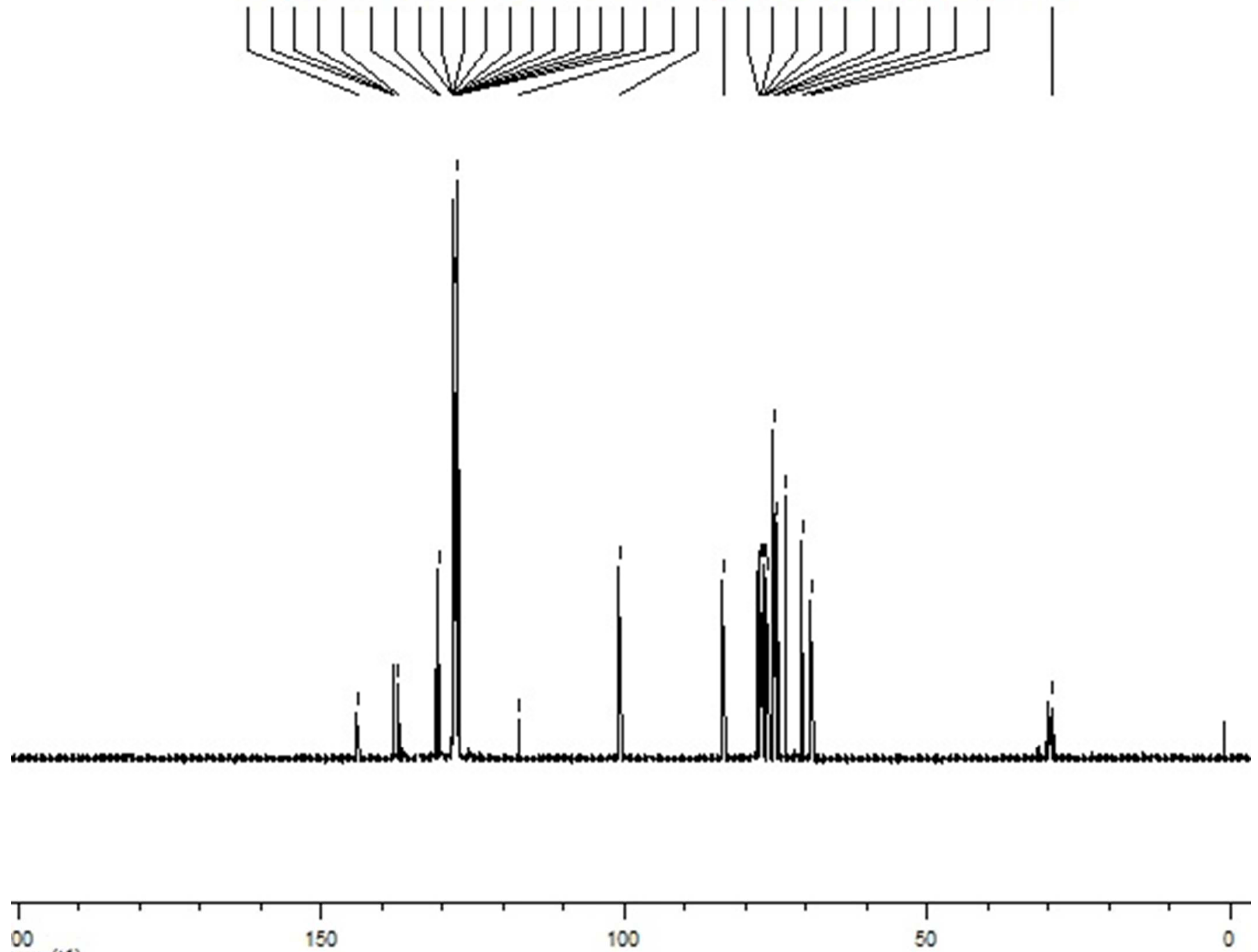
Figure S37. IR spectrum of complex1, $(a \mathrm{NHC}) \mathrm{Fe}(\mathrm{CO})_{4}$<smiles></smiles>

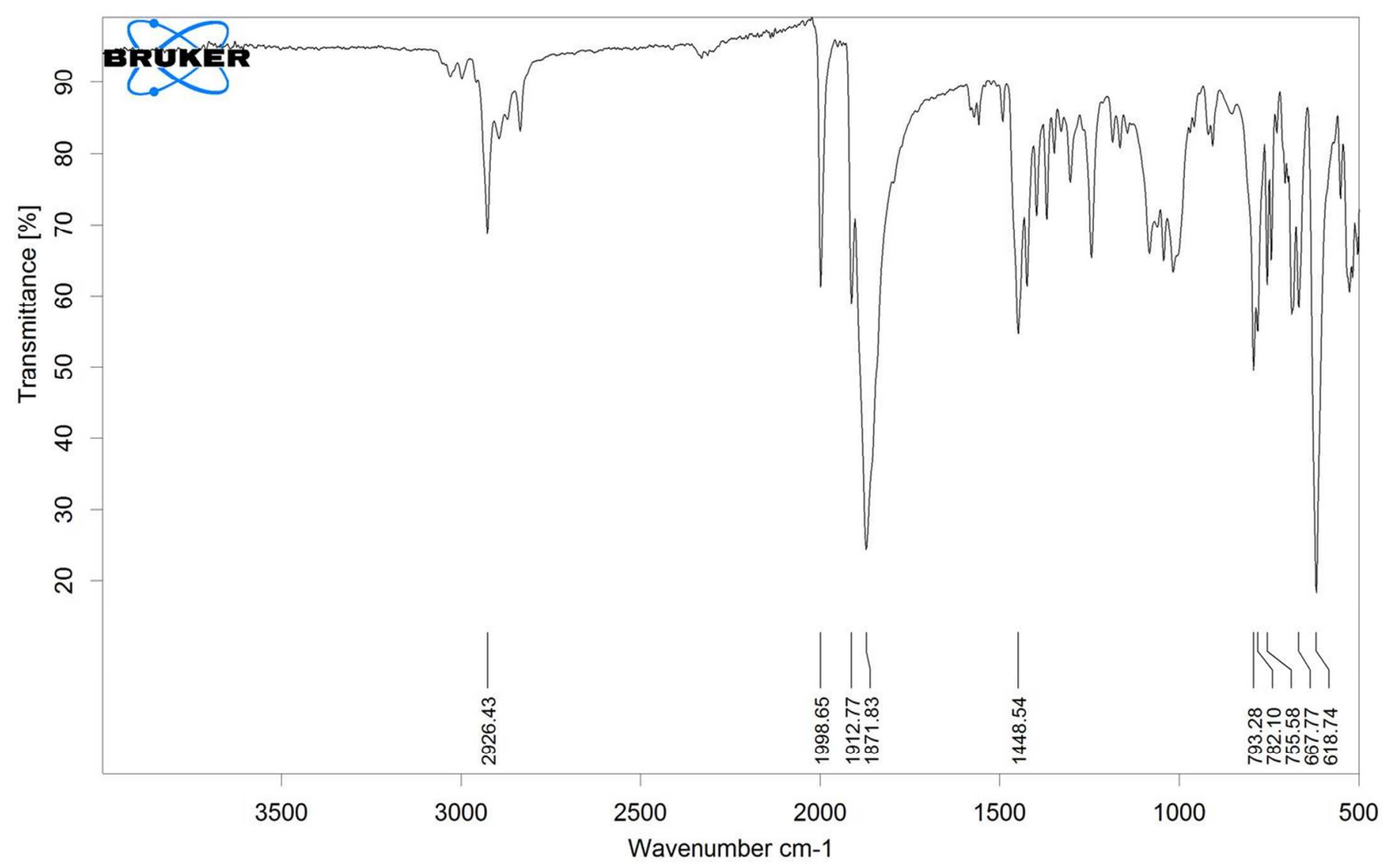


Figure S38. IR spectrum of complex 9
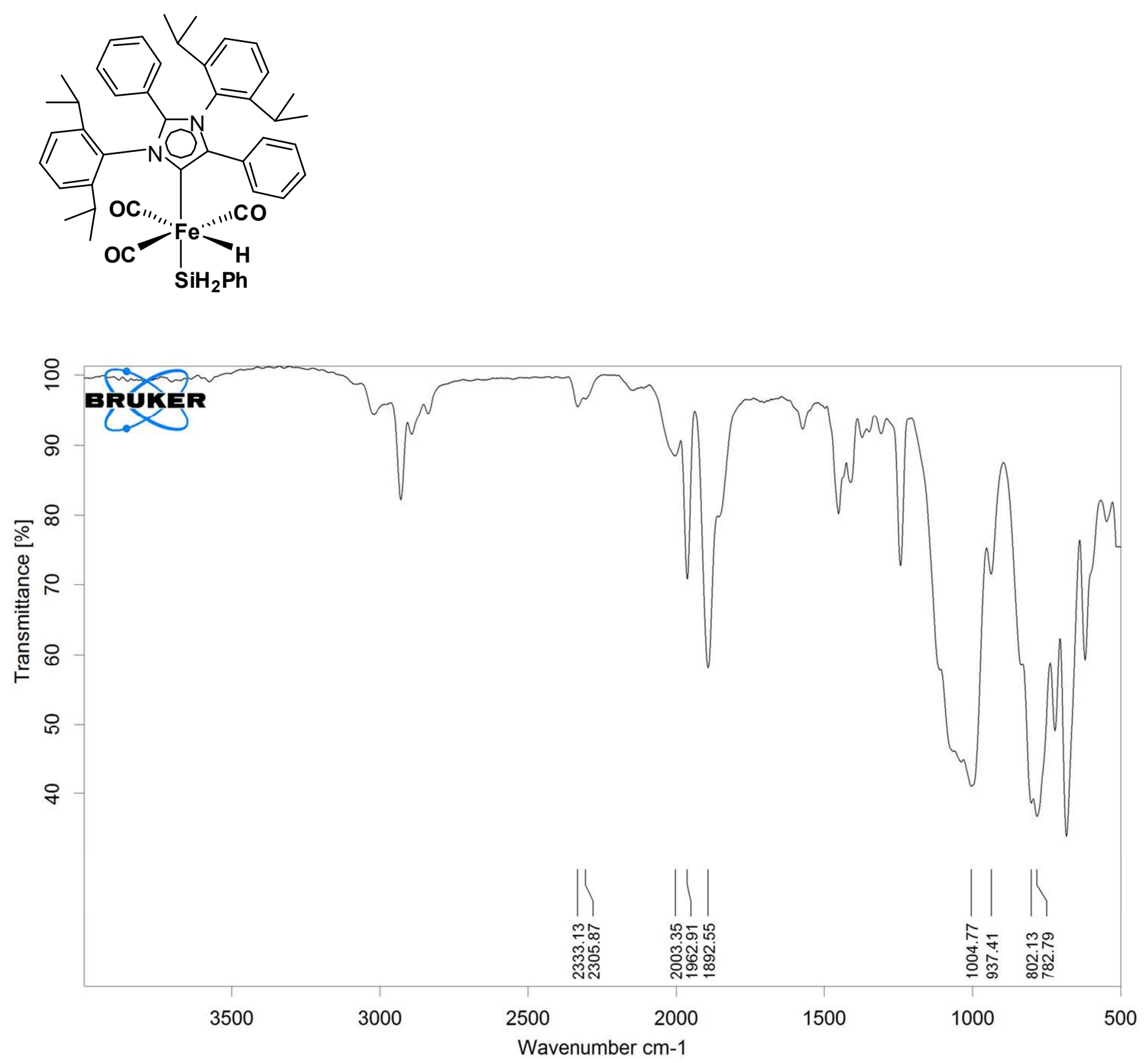

D:IFTIRIDr. S. MandalIMRINALIIron Hydride3.0 


\section{Computational details:}

All calculations were carried out using Density Functional Theory as implemented in the Gaussian $09^{8}$ quantum chemistry programs. The geometries of stationary points and transition states (TS) were optimized with the generalized gradient approximation (GGA) by means of the Becke exchange functional along with Lee, Yang, Parr correlation functional (LYP). ${ }^{9-12}$ We used double- $\zeta$ basis set with the relativistic effective core potential of Hay and Wadt $\left(\right.$ LANL2DZ) ${ }^{13-14}$ for the iron atom and 6-31G(d) basis set for other elements $(\mathrm{H}, \mathrm{C}, \mathrm{O}, \mathrm{N}$ and $\mathrm{Si})$. The geometries were optimized without any symmetry constraints. Harmonic force constants were computed at the optimized geometries to characterize the stationary points as minima or saddle points. The zero-point vibrational corrections were determined from the harmonic vibrational frequencies to convert the total energies $E_{e}$ to $E_{o}$. The rigid-rotor harmonic-oscillator approximation was applied for evaluating the thermal and entropic contribution that were necessary to obtain the enthalpies, $\mathrm{H}_{298}$ and Gibbs free energies, $\mathrm{G}_{298}$ at $298 \mathrm{~K}$. All transition states were located using the linear synchronous transit (LST) method ${ }^{15}$ which relies on the reaction coordinate to be kept fixed at particular distances while all other degrees of freedom relaxed. After the linear transit search, the transition state was optimized using the default Berny algorithm implemented in Gaussian 09 code. To get accurate energies of the optimized structures were reevaluated by additional single point energy calculations of each optimized geometry using 6-311++G** triple- $\zeta$ basis set ${ }^{16}$ for light atoms $(\mathrm{H}, \mathrm{C}, \mathrm{N}, \mathrm{O}, \mathrm{Si})$ and LANL2TZ(f) ${ }^{13-14}$ basis set for iron incorporated in the Gaussian program suites.

To decrease the computational cost, the isopropyl groups in the phenyl substituents in $a \mathrm{NHC}$ ligand were replaced with hydrogens and all the calculations were carried out with this truncated framework. We assume that such a small change in the steric environment would not significantly change the electronic environment of the metal complex and the chemistry described with these. 


\section{References:}

[1] Misal Castro, L. C.; Sortais, J.-B.; Darcel, C. Chem. Commun. 2012, 48, 151-153.

[2] Li, B.; Sortais, J.-B.; Darcel, C.; Dixneuf, P. H. ChemSusChem 2012, 5, 396 - 399.

[3] Hill, D. C.; Flugge, L. A.; Petillo, P. A. J. Org. Chem. 1997, 62, 4864-4866.

[4] Dolomanov, O. V.; Bourhis, L. J.; Gildea, R. J.; Howard, J. A. K.; Puschmann, H. OLEX2: a complete structure solution, refinement and analysis program. J. Appl. Cryst. 2009, 42, 339-341.

[5] SUPERFLI P, J. Appl. Cryst. 2007, 40, 786-790.

[6] SHELXL, Sheldrick, G. M. Acta Cryst. 2008, A64, 112-122.

[7] Barbour, L. J.; X-Seed, Graphical Interface to SHELX-97 and POV-Ray; University of Missouri; Columbia: Columbia, MO, 1999.

[8] Frisch, M. J.; Trucks, G. W.; Schlegel, H. B.; Scuseria, G. E.; Robb, M. A.; Cheeseman, J. R.; Scalmani, G.; Barone, V.; Mennucci, B.; Petersson, G. A.; Nakatsuji, H.; Caricato, M.; Li, X.; Hratchian, H. P.; Izmaylov, A. F.; Bloino, J.; Zheng, G.; Sonnenberg, J. L.; Hada, M.; Ehara, M.; Toyota, K.; Fukuda, R.; Hasegawa, J.; Ishida, M.; Nakajima, T.; Honda, Y.; Kitao, O.; Nakai, H.; Vreven, T.; Montgomery, J. A.; Peralta, Jr., J. E.; Ogliaro, F.; Bearpark, M.; Heyd, J. J.; Brothers, E.; Kudin, K. N.; Staroverov, V. N.; Kobayashi, R.; Normand, J.; Raghavachari, K.; Rendell, A.; Burant, J. C.; Iyengar, S. S.; Tomasi, J.; Cossi, M.; Rega, N.; Millam, J. M.; Klene, M.; Knox, J. E.; Cross, J. B.; Bakken, V.; Adamo, C.; Jaramillo, J.; Gomperts, R.; Stratmann, R. E.; Yazyev, O.; Austin, A. J.; Cammi, R.; Pomelli, C.; Ochterski, J. W.; Martin, R. L.; Morokuma, K.; Zakrzewski, V. G.; Voth, G. A.; Salvador, P.; Dannenberg, J. J.; Dapprich, S.; Daniels, A. D.; Farkas, Ö.; Foresman, J. B.; Ortiz, J. V.; Cioslowski, J.; Fox, D. J.; Gaussian 09, Gaussian Inc.,Wallingford, CT, 2010.

[9] Becke, A. D. Phys. Rev. A 1988, 38, 3098-3100.

[10] Becke, A. D. J. Chem. Phys. 1993, 98, 5648-5652.

[11] Vosko, S. H.; Wilk, L.; Nusair, M. Can. J. Phys. 1980, 58, 1200-1211.

[12] Lee, C.; Yang, W.; Parr, R. G. Phys. Rev. B: Condens. Matter Mater. Phys. 1988, 37, 785789.

[13] Hay, P. J.; Wadt,W. R. J. Chem. Phys. 1985, 82, 270-283.

[14] Wadt, W. R.; Hay, P. J. J. Chem. Phys. 1985, 82, 284-298.

[15] Helgren, T. A.; Lipscomb, W. N. Chem. Phys. Lett. 1977, 49, 225-232.

[16] Weigend, F.; Ahlrichs, R. Phys.Chem. Chem. Phys. 2005, 7, 3297-3305. 